\title{
ON THE MODULI SPACE OF SU $(n)$ MONOPOLES AND HOLOMORPHIC MAPS TO FLAG MANIFOLDS
}

\author{
BENJAMIN M. MANN \& R. JAMES MILGRAM
}

\begin{abstract}
In this paper, motivated by questions in mathematical physics, we study the geometry of the components in the spaces of based holomorphic maps from the Riemann sphere to complex flag manifolds, which we denote by $\operatorname{Rat}_{C}(\mathbb{F}(J))$. We decompose these spaces into smooth, in fact, complex strata each having a complex normal bundle. Using a modification of this filtration we study the forgetful map $l_{*}: H_{*}\left(\operatorname{Rat}_{C}(\mathbb{F}(J)) \rightarrow H_{*}\left(\Omega^{2} \mathbb{F}(J)\right)\right.$ and prove an Atiyah-Jones type stability theorem. We also use the filtrations to determine the basic groups $H_{*}\left(\operatorname{Rat}_{\overrightarrow{1}}(\mathbb{F}(J)) ; \mathbb{Z}\right)$ and show that $l_{*}$ has a nontrivial kernel for general flag manifolds.
\end{abstract}

\section{Introduction}

Let $S^{2}=\mathbb{C P}(1)$ denote the Riemann sphere, and $\mathbb{F}(J)$ the flag manifold of all sequences of complex $j_{i}$-dimensional planes through the origin in $\mathbb{C}^{n}$ for a fixed sequence $J=\left(0<j_{1}<\cdots<j_{m}<n\right)$. Thus, a point in $\mathbb{F}(J)$ is given by the flag

$$
\{0\} \subset \mathbb{V}^{j_{1}} \subset \cdots \subset \mathbb{V}^{j_{m}} \subset \mathbb{C}^{n},
$$

where $\mathbb{V}^{j_{i}}$ is a complex $j_{i}$-dimensional plane through the origin. We denote the space of based holomorphic maps of the Riemann sphere to the complex flag manifold $\mathbb{F}(J)$ by $\operatorname{Rat}(\mathbb{F}(J))$, and denote its natural inclusion into $\Omega^{2} \mathbb{F}(J)$ given by forgetting the complex structure by

$$
l(J): \operatorname{Rat}(\mathbb{F}(J)) \rightarrow \Omega^{2} \mathbb{F}(J) .
$$

The $\operatorname{Rat}(\mathbb{F}(J))$ spaces occur naturally in at least two distinct contexts: first, as moduli spaces of SU(n)-monopoles [8], [9], [13] and second, when $m=1$, as moduli spaces of linear control equations. Additionally, it had originally been hoped when we started this work that understanding these spaces would lead to a better understanding of the instanton moduli spaces. Indeed, our recent proof of the Atiyah-Jones conjecture [2] is

Received August 15, 1991 and, in revised form, April 7, 1992. Both authors were partially supported by grants from the National Science Foundation. 
motivated by the methods of geometrically decomposing the moduli spaces of holomorphic maps considered here.

We studied these spaces when $m=1$ in [11] and, using some ideas from linear control theory, were able to determine both their geometry and cohomology. In this paper we study these spaces for arbitrary $m$. Just as in [11] we obtain complete control of the geometry of $\operatorname{Rat}(\mathbb{F}(J))$. This analysis is presented in the first six sections. These results are then applied in $\S 7$ to give one of our main results, the following stability theorem for general flag manifolds.

Theorem A. Let $\mathbb{F}(J)$ be an arbitrary flag manifold. Given $k>0$ there is an $m$-tuple of positive integers $\left(s_{1}(k), \cdots, s_{m}(k)\right)$ so that for any $C$ with $c_{i} \geq s_{i}(k)$ then the natural inclusion

$$
\imath(C, J): \operatorname{Rat}_{C}(\mathbb{F}(J)) \rightarrow \Omega_{C}^{2} \mathbb{F}(J)
$$

is a homology equivalence through dimension at least $k$. If $J$ is not $(1,2, \cdots, m)$, representing the complete flag of length $n=m+1$, then both $\operatorname{Rat}_{C}(\mathbb{F}(J))$ and $\Omega^{2} \mathbb{F}(J)$ are simply connected so that $l(C, J)$ is then a homotopy equivalence through the same range.

Corollary B. Let $\mathbb{F}(J)$ be a flag manifold where $j_{i+1}-j_{i}>1$ for all $i$. Then the range of stability in Theorem $\mathrm{A}$ is $k=2 c+1$ where $c=\min _{i}\left(c_{i}\right)$.

This corollary follows immediately from our proof of Theorem A and our sharp bound for the stability theorem for the Grassmannian case [11], [12]. There is a similar corollary using the sharp bound of [5] when at least one of the gaps $j_{i+1}-j_{i}=1$.

Theorem $\mathrm{A}$ is essentially Corollary 7.9 of $\S 7$ below. Earlier, special cases of this stability theorem were proved by Segal [14] when $\mathbb{F}(J)$ is a projective space, by Kirwan [10] when $\mathbb{F}(J)$ is a Grassmannian, and by Guest [7] when $\mathbb{F}\left(J_{m}\right)$ is a complete flag of the form $J_{m}=(1,2, \cdots, m)$, i.e. $j_{i+1}-j_{i}=1$ for $1 \leq i<m$ but $m$ is not required to be $n-1$. For a more detailed summary of the history of these stability theorems see [12].

Theorem A and Corollary B give the complete stability theorem for holomorphic mapping spaces from the Riemann sphere to the classical complex flag manifolds, but there is one important generalization worth mentioning. One can consider holomorphic maps from the Riemann sphere to $G / P$ where $G$ is a complex semisimple Lie group and $P$ is a parabolic subgroup. In discussions with $\mathrm{C}$. Boyer and J. Hurtubise it has recently become clear that the methods developed here and in [2] will extend to cover this most general case.

Combining Theorem A with the work of Hurtubise and Murray [8], [9], which shows that the $\operatorname{Rat}(\mathbb{F}(J))$ spaces for full flags $J$ are equivalent 
to the spaces of "maximal symmetry breaking" $\mathrm{SU}(n)$ monopoles, we obtain a version of the Atiyah-Jones conjecture for instantons in these cases. Also, Murray [13] has given a stratification of the monopole moduli space which is different than the one we give here for $\operatorname{Rat}(\mathbb{F}(J))$ and it should be worthwhile to compare the two.

In $\S \S 8-10$ we apply the geometric techniques developed in the first six sections to analyze some basic examples, namely, $\operatorname{Rat}_{\hat{1}}(\mathbb{F}(J))$ for general $J$ flags. It is at this point that the fundamental complexity of the topology of flag versus Grassmannian Rats becomes apparent. $\operatorname{Rat}_{1}\left(\mathbb{G}_{n, m}\right)$ has the homotopy type of the complement of the zero section of a vector bundle over complex projective space, $\S 8.6$, but $\operatorname{Rat}_{\overrightarrow{1}}(\mathbb{F}(J))$ is much more complicated and difficult to describe. In $\S \S 9$ and 10 we first determine the homology of the individual strata and then, in principle, the resulting homology groups for the $\operatorname{Rat}_{\overrightarrow{1}}(\mathbb{F}(J))$ themselves. In particular, the results are given explicitly for the spaces $\operatorname{Rat}_{1,1}\left(\mathbb{F}\left(j_{1}, j_{2} ; n\right)\right)$ of 2-stage flags, $\mathbb{C}^{j_{1}} \subset \mathbb{C}^{j_{2}} \subset \mathbb{C}^{n}$.

Finally, in $\S 11$ we discuss the homology of arbitrary flag manifolds above the range of stability given by Theorem $\mathrm{A}$ and see new phenomena not present in the projective space [5] or Grassmannian [11] cases. That is, while

$$
l(n, m ; k)_{*}: H_{*}\left(\operatorname{Rat}_{k}\left(\mathbb{G}_{n, m}\right)\right) \rightarrow H_{*}\left(\Omega_{k}^{2}\left(\mathbb{G}_{n, m}\right)\right)
$$

is always an injection from [11], it is not true that $l(J ; C)$ induces an injection in homology for arbitrary flags. We conclude this paper with examples where $l(J ; C)$ has a kernel. Indeed, we conjecture that $l(J ; C)$ always has a nontrivial kernel whenever $J$ involves flags of length at least 2. Since this is tied to the structure of $\Omega^{2} \mathbb{F}(J)$, we begin $\S 11$ by computing $H_{*}\left(\Omega^{2} \mathbb{F}(J)\right)$ and then compare the answer to the results of $\S \S 7-10$ to construct our kernel classes.

Now we describe in more detail the content of the first seven sections.

In [5] and [11] holomorphic maps into projective spaces and Grassmann manifolds were analyzed. In [11] we analyzed $\operatorname{Rat}_{k}\left(\mathbb{G}_{n, m}\right)$ by constructing a stratification of the entire space by smooth manifolds (each with a trivial normal bundle, and each contained in the closure of higher-dimensional strata) whose homotopy types we understood. These strata were then organized into a filtration, and the resulting spectral sequence was shown to collapse. For general flag manifolds the plan of attack is quite similar. Again, using the techniques developed in [11], we construct a stratification of $\operatorname{Rat}_{C}(\mathbb{F}(J))$ by smooth manifolds, each with a complex, and hence 
orientable, normal bundle, and each contained in the closure of higherdimensional strata. However, the details are quite a bit more complex in this new case and the basic stratification of $\$ 7$ does not have much in common with that of [11].

Our main effort in the first half of this paper is to describe the geometry of these strata and the way in which they couple together to give the spaces $\operatorname{Rat}_{C}(\mathbb{F}(J))$. These strata also can be naturally organized to give filtrations of the $\operatorname{Rat}_{C}(\mathbb{F}(J))$ 's. This leads to spectral sequences whose $E^{1}$ and $E^{2}$ terms are described in $\S \S 6$ and 7.

First we generalize the method of [11] used to describe the points of $\operatorname{Rat}_{C}(\mathbb{F}(J))$.

Theorem C. There is a one-to-one correspondence between elements of $\operatorname{Rat}_{C}(\mathbb{F}(J))$ and m-tuples

$$
\left(\left[D_{1}, N_{1}\right], \cdots,\left[D_{m}, N_{m}\right]\right),
$$

where the pairs $\left[D_{r}, N_{r}\right]$ independently run through a prescribed normal form of relatively prime polynomial matrices satisfying additional constraints (see (2.1) and $\S 4$ for the precise conditions).

These constraints are quite complicated to describe, involving vanishing conditions on linear combinations of products, but they lead to our precise determination of the dimensions and geometries of the various strata.

The remaining results need a bit more notation. The components of Rat $_{C}(\mathbb{F}(J))$ are indexed by sequences of nonnegative integers $C=\left(c_{1}, \ldots\right.$, $c_{m}$ ) corresponding to the total Chern class of the holomorphic map. For any fixed total Chern class $C$ we consider all sequences of $\frac{m(m+1)}{2}$ nonnegative integers given by

$$
K=\left(k_{1}(1), k_{1}(2), k_{2}(2), \cdots, k_{1}(m), \cdots, k_{m}(m)\right),
$$

where the $k_{i}(j) \geq 0$ satisfy the recursive relations given in (3.14). In what follows we call each such $K$ a "multi-partition" of $C$.

The reduction of holomorphic maps to polynomial matrices gives manifold coordinates for a geometric decomposition of $\operatorname{Rat}_{C}(\mathbb{F}(J))$ into complex submanifolds whose connected components, which we denote by $Y(B)$, are indexed by the various multipartitions $K$ given in (1.2). The geometry of $\operatorname{Rat}_{C}(\mathbb{F}(J))$ and each individual strata is explained by the following theorem.

\section{Theorem D.}

1. $\operatorname{Rat}_{C}(\mathbb{F}(J))$ is a connected complex manifold of complex dimension $\sum c_{i}\left(j_{i+1}-j_{i-1}\right)$.

2. For each multipartition $K, Y(K)$ is a complex submanifold of Rat $_{C}(\mathbb{F}(J))$ of dimension $\sum_{1}^{n} c_{i}\left(j_{i+1}-j_{i}\right)+\sum_{i, s} k_{i}(s)\left(j_{i}-j_{i-1}\right)$. Further- 
more, the normal bundle $\nu(l(K))$ of $Y(K)$ in $\operatorname{Rat}_{C}(\mathbb{F}(J))$ is a complex bundle and hence orientable.

3. $Y(K)$ is the total space of an iterate fibration where each of the individual fibers or bases is homotopically a Grassmannian Rat space. (See (6.1) for the precise statement.)

In $\S 7$ we modify the $Y(K)$ filtration of $\S 6$ to obtain a second filtration compatible with the Serre filtration for the fibration

$$
\Omega^{2} \mathbb{G}_{j_{1}, j_{2}-j_{1}} \rightarrow \Omega_{C}^{2} \mathbb{F}(J) \rightarrow \Omega_{C^{\prime}}^{2} \mathbb{F}\left(J^{\prime}\right)
$$

induced by forgetting the smallest piece of the flag. This comparison, together with the main result of [11], leads directly to Theorem A.

We would like to thank J. Hurtubise for communicating to us both his and M. Murray's results on $\mathrm{SU}(n)$-monopoles and the referee for his careful reading of the paper and many helpful comments.

\section{The geometry of $\operatorname{Rat}_{k}\left(\mathbb{G}_{i, n-i}\right)$}

In this section we describe the decomposition, given in [11], of $\operatorname{Rat}_{k}\left(\mathbb{G}_{i, n-i}\right)$ into smooth strata each with a trivial normal bundle in the entire space. This decomposition is basic to understanding the geometry of both $\operatorname{Rat}_{k}\left(\mathbb{G}_{i, n-i}\right)$ and $\operatorname{Rat}_{C}(\mathbb{F}(J))$. We begin by recalling a normal form for elements of $\operatorname{Rat}_{k}\left(\mathbb{G}_{i, n-i}\right)$.

Every rational map $f_{T}: \mathbb{C P}^{1} \rightarrow \mathbb{G}_{i, n-i}$ is represented by a pair of coprime, based matrices $T=(D(z), N(z))$ with coefficients in the polynomial ring $\mathbb{C}[z]$. As is standard we denote by $\mathbb{C}[z]$ the ring of polynomials in the field of rational functions $\mathbb{C}(z)$. Here $D(z) \in \operatorname{Mat}_{i, i}(\mathbb{C}[z])$ and $N(z) \in \operatorname{Mat}_{i, n-i}(\mathbb{C}[z])$, coprime means that there are matrices $A(z)$ and $B(z)$, which are $i \times i$ and $(n-i) \times i$ with entries in $\mathbb{C}[z]$, so that $D A+N B=I$, the $i \times i$ identity matrix, and based means that $\lim _{z \mapsto \infty}\left(D^{-1}(z) N(z)\right)=0$. In particular, $D(z)^{-1}$ exists if and only if $\operatorname{Det}(D(z)) \neq 0$ in $\mathbb{C}[z]$, and $(D(z), N(z)) \in \operatorname{Rat}_{k}\left(\mathbb{G}_{i, n-i}\right)$ if and only if $\operatorname{Det}(D(z))$ is a (monic) polynomial of degree $k$.

The fact that $D$ and $N$ are coprime implies that for every $z_{0} \in \mathbb{C}$ the rectangular matrix of $n$-row vectors $\left[D\left(z_{0}\right), N\left(z_{0}\right)\right]$ has rank $i$ and hence defines a unique $i$-plane in $\mathbb{C}^{n}$, namely the span of the row vectors. This gives the correspondence.

Note from the correspondence above that two such pairs, $(D(z), N(z))$, $\left(D^{\prime}(z), N^{\prime}(z)\right)$, are equivalent and represent the same element of $\operatorname{Rat}_{k}\left(\mathbb{G}_{i, n-i}\right)$ if and only if there is a matrix $U(z) \in \mathrm{GL}_{i}(\mathbb{C}[z])$ so that 
$U D=D^{\prime}, U N=N^{\prime}$. Note that $U \in \mathrm{GL}_{i}(\mathbb{C}[z])$ if and only if $U \in$ $\operatorname{Mat}_{i, i}(\mathbb{C}[z])$, and $\operatorname{Det}(U)$ is a nonzero constant.

In general, by multiplying on the left by a unimodular matrix $U$ we can bring $f_{T}$ to the following normal form (which is unique):

$$
\begin{aligned}
U \circ f_{T} & =U[D, N]=[P, Q] \\
& =\left[\begin{array}{ccccccc}
p_{11} & p_{12} & \cdots & p_{1 i} & q_{11} & \cdots & q_{1, n-i} \\
0 & p_{22} & \cdots & p_{2 i} & q_{21} & \cdots & q_{2, n-i} \\
\vdots & \vdots & \ddots & \vdots & \vdots & \ddots & \vdots \\
0 & 0 & \cdots & p_{i i} & q_{i 1} & \cdots & q_{i, n-i}
\end{array}\right],
\end{aligned}
$$

where

(a) $P$ is upper triangular with monic diagonal terms,

(b) degree $\left(p_{b a}\right)<\operatorname{degree}\left(p_{a a}\right)=k_{a}$ for $b<a$,

(c) $\sum_{l=1}^{i} k_{l}=c(f)=k$,

(d) degree $\left(q_{i b}\right)<\operatorname{degree}\left(p_{i i}\right)$,

(e) the basing condition on $f_{T}$ forces constraints on all the $q_{a b}$; see $[11, \S 3]$ for details.

We use this normal form to decompose $\operatorname{Rat}_{k}\left(\mathbb{G}_{i, n-i}\right)$ into smooth strata. Let $K=\left(k_{1}, \cdots, k_{i}\right)$ be a partition of $k$. That is, each $k_{a} \geq 0$ and $\sum_{a=1}^{i} k_{a}=k$. Lexicographically order the partitions of $k$ by setting $L=\left(l_{1}, \cdots, l_{i}\right)<K=\left(k_{1}, \cdots, k_{i}\right)$ if $l_{i}<k_{i}$ or, if $l_{b}=k_{b}$ for $b>a$ then $l_{a}<k_{a}$.

Definition 2.2. For each partition of $k$ let

$X\left(k_{1}, \cdots, k_{i}\right)=\left\{\begin{array}{l|l}f_{T} \in \mathrm{Rat}_{k}\left(\mathbb{G}_{i, n-i}\right) & \begin{array}{l}\text { The normal form of } T=[P, Q] \\ \text { satisfies degree }\left(p_{a a}\right)=k_{a}\end{array}\end{array}\right\}$.

The following is Proposition 4.2 of [11].

Proposition 2.3. $X\left(k_{1}, \cdots, k_{i}\right)$ is a complex submanifold of $\operatorname{Rat}_{k}\left(\mathbb{G}_{i, n-i}\right)$ of complex dimension $(n-i+1) k+\sum_{a=2}^{i}(a-1) k_{a}$.

Associated to each $k$, there is a filtration of $\operatorname{Rat}_{k}\left(\mathbb{G}_{i, n-i}\right)$ by open manifolds,

$$
\mathscr{F}_{r}\left[\operatorname{Rat}_{k}\left(\mathbb{G}_{i, n-i}\right)\right]=\bigcup_{k_{i} \geq r} X\left(k_{1}, \cdots, k_{i}\right) .
$$

To reassemble $\operatorname{Rat}_{k}\left(\mathbb{G}_{i, n-i}\right)$ from this stratification one needs to know the structure of the natural inclusions

$$
\imath\left(k_{1}, \cdots, k_{i}\right): X\left(k_{1}, \cdots, k_{i}\right) \hookrightarrow \mathscr{F}_{k_{i}}\left[\operatorname{Rat}_{k}\left(\mathbb{G}_{i, n-i}\right)\right] .
$$

Proposition 2.5. The normal bundle $\nu\left(l\left(k_{1}, \cdots, k_{i}\right)\right)$ is trivial.

Details are given in $\S 4$ of [11]. 
Finally, in [11] the following result is also proved.

Proposition 2.6. There is a sequence of fibrations:

$$
\begin{aligned}
& \operatorname{Rat}_{k_{1}}(\mathbb{C P}(n-i)) \rightarrow X\left(k_{1}, \cdots, k_{i}\right) \\
& \operatorname{Rat}_{k_{2}}(\mathbb{C P}(n-i)) \rightarrow X\left(k_{2}, \cdots, k_{i}\right) \\
& \downarrow \pi_{2} \\
& \begin{array}{c}
\operatorname{Rat}_{k_{n-1}}(\mathbb{C P}(n-i)) \rightarrow \quad X\left(k_{i-1}, k_{i}\right) \\
\operatorname{Rat}_{k_{i}}(\mathbb{C P}(n-i)) \\
\stackrel{\mid \pi_{n-1}}{\rightarrow} \quad X\left(k_{i}\right) .
\end{array}
\end{aligned}
$$

It is shown in [11] that the homology and cohomology of the space $X\left(k_{1}, \cdots, k_{i}\right)$ in (2.7) is just the tensor product of the homology of the successive fibers (with field coefficients) when $i \leq n-i$.

Next we filter $\mathbb{G}_{i, n-i}$ by first using the lexicographic ordering on the $X\left(k_{1}, \cdots, k_{i}\right)$ and then refining further to include the filtrations described above on each $X\left(k_{1}, \cdots, k_{i}\right)$. This is not a problem since we are dealing here with finite-dimensional spaces and consequently the total number of filtering subspaces at each stage can be assumed to be finite. The result is a spectral sequence for the space $\operatorname{Rat}_{k}\left(\mathbb{G}_{i, n-i}\right)$. Specifically we have

Theorem 2.8 [11]. There is a spectral sequence with $E_{2}$ term

$$
\bigoplus_{K} H^{*}\left(\Sigma^{2 t(K)}\left\{\operatorname{Rat}_{k_{1}}(\mathbb{C P}(n-i)) \times \cdots \times \operatorname{Rat}_{k_{i}}(\mathbb{C P}(n-i))\right\} ; \mathbb{F}\right)
$$

converging to $H^{*}\left(\operatorname{Rat}_{k}\left(\mathbb{G}_{i, n-i}\right) ; \mathbb{F}\right)$, where $\mathbb{F}$ is a field, $K$ runs over all partitions $\left(k_{1}, \cdots, k_{i}\right)$ of $k$ having length $i$, and $t(K)=(i-1) k-$ $\sum_{a=2}^{i}(a-1) k_{a}$. If $i \leq n-i$ then the spectral sequence collapses to $E_{2} \cong E_{\infty}$ in this case.

This collapsing for Grassmannians is very special and turns out not to be true for the more general flag manifolds.

\section{Normal forms for flag manifolds: preliminaries}

In the next two sections we establish a normal form for elements of $\operatorname{Rat}_{C}(\mathbb{F}(J))$ which generalizes the normal form for elements of $\operatorname{Rat}_{k}\left(\mathbb{G}_{i, n-i}\right)$ given in [11]. In particular we prove the precise version of Theorem $B$ in 
the introduction. The main difficulty will be in properly interpreting the basing condition for elements of $\operatorname{Rat}_{C}(\mathbb{F}(J))$. For $f \in \operatorname{Rat}_{C}(\mathbb{F}(J))$ with $C=\left(c_{1}, \cdots, c_{m}\right)$ we have

$$
f(z)=\left\{\mathbb{V}^{j_{1}}(z) \subset \cdots \subset \mathbb{V}^{j_{m}}(z) \subset \mathbb{C}^{n}\right\} .
$$

For each $j_{i}, 1 \leq i \leq m$, the projection

$$
\left(p_{i}(f)\right)(z)=p_{i}(f(z))=\left\{\mathbb{V}^{j_{i}}(z) \subset \mathbb{C}^{n}\right\}
$$

defines an element $p_{i}(f) \in \operatorname{Rat}_{c_{i}}\left(\mathbb{G}_{j_{i}, n-j_{i}}\right)$. Notice that this assignment makes use of the basing condition $f(\infty)=\left\{\mathbb{C}^{j_{1}} \subset \mathbb{C}^{j_{2}} \subset \cdots \subset \mathbb{C}^{j_{m}} \subset \mathbb{C}^{n}\right\}$. In particular, we have an embedding

$$
\prod_{1}^{m} p_{i}: \operatorname{Rat}_{C}(\mathbb{F}(J)) \hookrightarrow \prod_{1}^{m} \operatorname{Rat}_{c_{i}}\left(\mathbb{G}_{j_{i}, n-j_{i}}\right) .
$$

We now discuss this image in more detail. We begin with the largest subspace $\mathbb{V}^{j_{m}}(z) \subset \mathbb{C}^{n}$, which, as in (2.1), we represent by

$$
p_{m}(f(z))=\left[P_{m}(z), Q_{m}(z)\right] \text {. }
$$

Here $P_{m}(z)$ is in reduced form, $(2.1)(\mathrm{a}),(\mathrm{b}),(\mathrm{c})$, and $\left[P_{m}(z), Q_{m}(z)\right]$ is the preferred explicit representative for the based map $p_{m}(f) \in \operatorname{Rat}_{c_{i}}\left(\mathbb{G}_{j_{m}, n-j_{m}}\right)$ as given in [11]. In particular,

(a) $P_{m}(z), Q_{m}(z)$ are relatively prime,

(b) $P_{m}(z)$ is $j_{m} \times j_{m}$, and

(c) $Q_{m}(z)$ is $j_{m} \times\left(n-j_{m}\right)$.

Next, let $\left[P_{m-1}, Q_{m-1}\right]$ be the polynomial normal form representing $\mathbb{V}^{j_{m-1}}(z)$ in $\mathbb{C}^{n}$. Since $\mathbb{V}^{j_{m-1}}(z)$ is a subspace of $\mathbb{V}^{j_{m}}(z)$, it is clear that we can represent $\mathbb{V}^{j_{m-1}}(z)$ by

$$
[E, F]\left[P_{m}, Q_{m}\right]=\left[P_{m-1}, Q_{m-1}\right]
$$

for some relatively prime pair of rational matrices $[E, F]$.

Lemma 3.5. The matrices $[E, F]$ in (3.4) are polynomial.

Proof. Since $\left[P_{m}, Q_{m}\right]$ is in normal form, $P_{m}$ and $Q_{m}$ are relatively prime. Hence, there exist polynomial matrices $X$ and $Y$ such that $P_{m} X+$ $Q_{m} Y=I$. But then

$$
[E, F]=[E, F]\left[P_{m}, Q_{m}\right]\left[\begin{array}{c}
X \\
Y
\end{array}\right]=\left[P_{m-1}, Q_{m-1}\right]\left[\begin{array}{c}
X \\
Y
\end{array}\right]
$$

and the right-hand side is manifestly polynomial. q.e.d. 
Left multiplication by a suitable unimodular matrix will convert the correcting term $[E, F]$ to $[U E, U F]=\left[D_{m-1}(z), N_{m-1}(z)\right]$ with $D_{m-1}(z)$ as in $(2.1)(\mathrm{a}),(\mathrm{b}),(\mathrm{c})$. The fact that $E$ and $F$ are relatively prime implies that $D_{m-1}(z)$ and $N_{m-1}(z)$ are also relatively prime. However, there is no reason to suppose that

$$
\left[D_{m-1}(z), N_{m-1}(z)\right] \in \mathrm{Rat}_{c_{m-1}}\left(\mathbb{G}_{j_{m-1}, j_{m}-j_{m-1}}\right)
$$

since there is no reason to assume that $\left[D_{m-1}(z), N_{m-1}(z)\right]$ is properly based.

Example 3.6. Consider the element $f \in \operatorname{Rat}_{(2,2)}(\mathbb{F}(1,2,5))$ whose image under the embedding given in (3.2) is

$$
\begin{aligned}
& p_{1}(f)=\left(z^{2}-2,3(z-1), 1,2,1\right) \in \operatorname{Rat}_{2}\left(\mathbb{G}_{1,4}\right), \\
& p_{2}(f)=\left(\begin{array}{ccccc}
z^{2}-2 & 0 & 1 & 2 & 1 \\
0 & 3 & 0 & 0 & 0
\end{array}\right) \in \operatorname{Rat}_{2}\left(\mathbb{G}_{-\{2,3\}}\right) .
\end{aligned}
$$

While $p_{1}(f)$ and $p_{2}(f)=\left[P_{2}, Q_{2}\right]$ are both based maps, notice that $[E, F]=\left[D_{1}, N_{1}\right]=[1, z-1]$ does not satisfy the Grassmannian basing condition. Nonetheless, as we shall see throughout the remainder of the paper, in most cases it will be better to think of this element $f$ in terms of the pair $\left(\left[D_{1}, N_{1}\right],\left[P_{2}, Q_{2}\right]\right)$ rather than in terms of the associated image in (3.2).

We reiterate. The based map is

$$
\begin{aligned}
p_{m-1}(f(z)) & =\left[\left(D_{m-1}(z), N_{m-1}(z)\right) P_{m}(z),\left(D_{m-1}(z), N_{m-1}(z)\right) Q_{m}(z)\right] \\
& =\left[P_{m-1}, Q_{m-1}\right] .
\end{aligned}
$$

From this point forward we will use the notation $\left[P_{i}, Q_{i}\right]$ to represent $p_{i}(f)$ while reserving $\left[D_{i}, N_{i}\right]$ for the polynomial matrix pair which multiplies $\left[P_{i+1}, Q_{i+1}\right]$ to obtain $\left[P_{i}, Q_{i}\right]$. We view $\left[D_{i}(z), N_{i}(z)\right]$ as describing the way in which $V^{j_{i}}(z)$ is contained in $V^{j_{i+1}}(z)$, while $\left[P_{i}(z), Q_{i}(z)\right]$ describes the way $V^{j_{1}}(z)$ is contained in $\mathbb{C}^{n}$.

Thus, the map described by (3.7) must satisfy the following:

(a) $p_{m}(f(z))=\left[P_{m}(z), Q_{m}(z)\right]$ is in the normal form given in (2.1) (see (3.3)).

(b) $p_{m-1}(f(z))=\left[P_{m-1}(z), Q_{m-1}(z)\right]$ is in the normal form given in (2.1) (see (3.7))

(c) $D_{m-1}$ is $j_{m-1}$ by $j_{m-1}$.

(d) $N_{m-1}$ is $j_{m-1}$ by $j_{m}-j_{m-1}$.

(e) While each $[P, Q]$ factor is in normal form, and each $D$ matrix can be brought to normal form, (3.7) and the basing condition place further 
conditions on the pair $\left[D_{m-1}, N_{m-1}\right]$. We shall examine this in more detail in what follows.

(f) Recall that $\left[P_{m}, Q_{m}\right]$ and $\left[D_{m-1}, N_{m-1}\right]$ are each pairwise relatively prime. In fact, more is true as the next lemma shows.

Lemma 3.8. The condition that two polynomial factors of $p_{m-1}(f(z))$,

$$
P_{m-1}=\left(D_{m-1}(z), N_{m-1}(z)\right) P_{m}(z)
$$

and

$$
Q_{m-1}=\left(D_{m-1}(z), N_{m-1}(z)\right) Q_{m}(z)
$$

are relatively prime is equivalent to the condition that $D_{m-1}$ and $N_{m-1}$ are relatively prime.

Proof. Recall by assumption that $P_{m}$ and $Q_{m}$ are relatively prime. If $D_{m-1}$ and $N_{m-1}$ are relatively prime then there exist polynomial matrices $X, X^{\prime}, Y$, and $Y^{\prime}$ such that $P_{m} X+Q_{m} Y=I$ and $D_{m-1} X^{\prime}+N_{m-1} Y^{\prime}=$ $I$. Now just expand

$$
\left[D_{m-1}, N_{m-1}\right]\left[P_{m}, Q_{m}\right]\left[\begin{array}{c}
X \\
Y
\end{array}\right]\left[\begin{array}{c}
X^{\prime} \\
Y^{\prime}
\end{array}\right] .
$$

Conversely, if $P_{m-1}$ and $Q_{m-1}$ are relatively prime then there exist polynomial matrices $W$ and $Z$ such that $P_{m-1} W+Q_{m-1} Z=I$. It follows that

$$
\begin{aligned}
{\left[D_{m-1}, N_{m-1}\right]\left[P_{m} W+Q_{m} Z\right] } & =\left[D_{m-1}, N_{m-1}\right]\left[P_{m}, Q_{m}\right]\left[\begin{array}{c}
W \\
Z
\end{array}\right] \\
& =\left[P_{m-1}, Q_{m-1}\right]\left[\begin{array}{c}
W \\
Z
\end{array}\right]=I .
\end{aligned}
$$

Remark 3.9. Lemma 3.8 gives an independent proof that the $[E, F]$ matrices of (3.4) (which are integral by Lemmas 3.5) are relatively prime. Thus, not only do $p_{m}(f)$ and $p_{m-1}(f)$ uniquely determine $\left[D_{m-1}, N_{m-1}\right]$ up to normal form but given $p_{m}(f)$ and any relatively prime pair $\left[D_{m-1}\right.$, $N_{m-1}$ ] their product yields a well-defined holomorphic map $p_{m-1}(f)$ from $S^{2}$ to $\mathbb{G}_{j_{m-1}, n-j_{m-1}}$. Lastly, as mentioned in Example 3.6, we are interested in all choices of $\left[D_{m-1}, N_{m-1}\right]$ so that $p_{m-1}(f)$ is a based map.

Continuing inductively we may represent $\mathbb{V}^{j_{r}}(z)$ in $\mathbb{C}^{n}$ by

$$
\begin{aligned}
p_{r}(f(z)) & =\left[\left(D_{r}(z), N_{r}(z)\right) P_{r+1}(z),\left(D_{r}(z), N_{r}(z)\right) Q_{r+1}(z)\right] \\
& =\left[P_{r}, Q_{r}\right]
\end{aligned}
$$

where

(a) $p_{r+1}(f(z))=\left[P_{r+1}(z), Q_{r+1}(z)\right]$ is inductively defined by (3.3), (3.7) and iterations of $(3.10)$ for $m>r+1$, 
(b) each $p_{j}(f)$, for $r \leq j \leq m$, is in the Grassmannian based normal form given in (2.1),

(c) $D_{r}$ is $j_{r}$ by $j_{r}$,

(d) $N_{r}$ is $j_{r}$ by $j_{r+1}-j_{r}$,

(e) the two polynomial factors of $p_{r}(f), P_{r}$ and $Q_{r}$, are relatively prime (this follows by iterating Lemma 3.8),

(f) again, while each $\left[P_{j}, Q_{j}\right]$ factor is in normal form, and each $D$ matrix can be brought to normal form by using unimodular matrices on the left, (3.10) and the basing conditions place further conditions on the $D_{j}$ and $N_{j}$.

(3.10)(e) is equivalent to the fact that $\left[P_{r}, Q_{r}\right]$ has maximal rank and hence represents a holomorphic map into the appropriate Grassmannian. That is, iterate applications of Lemma 3.8 guarantee that all the $p_{r}(f)=$ $\left[P_{r}, Q_{r}\right]$ matrices of $(3.3),(3.7)$, and $3.10(\mathrm{r})$ for $1 \leq r \leq m$ taken together represent a holomorphic map into the flag manifold precisely when each pair $\left[D_{j}, N_{j}\right]$ has the property that $D_{j}$ and $N_{j}$ are relatively prime for all $1 \leq j \leq m$.

Hence, we have shown that every element $f(z) \in \operatorname{Rat}_{C}(\mathbb{F}(J))$ can be written in the form

$$
\left(\left[D_{1}(z), N_{1}(z)\right], \cdots,\left[D_{m}(z), N_{m}(z)\right]\right),
$$

where $\left[D_{m}, N_{m}\right]=\left[P_{m}, Q_{m}\right]$, for suitable choices of $D_{i}$ and $N_{i}$. The $m$ tuple given in (3.11) represents the holomorphic map whose image under (3.2) we write as

$$
z \rightarrow \prod_{1}^{m} p_{i}(f)(z)=\left(\left[P_{1}(z), Q_{1}(z)\right], \cdots,\left[P_{m}(z), Q_{m}(z)\right]\right) .
$$

However, as Example 3.6 shows, we cannot expect each individual $[D, N]$ pair occurring in (3.11) to be described simply by the Grassmannian basing conditions of [11].

We shall see that elements of the form given in (3.11) are naturally classified by the diagonal degree pattern of the $D_{i}$ matrices. In the process of analyzing the basing conditions associated to these individual degree patterns certain essential building blocks which enable us to understand the topology of the $\operatorname{Rat}_{C}(\mathbb{F}(J))$ will appear.

To proceed we write

$$
D_{s}=\left(\begin{array}{cccc}
D_{1,1}(s) & E_{1,2}(s) & \cdots & E_{1, s}(s) \\
0 & D_{2,2}(s) & \cdots & E_{2, s}(s) \\
\vdots & \vdots & \ddots & \vdots \\
0 & 0 & \cdots & D_{s, s}(s)
\end{array}\right) \text {, }
$$


where $D_{i, i}(s)$ is a $\left(j_{i}-j_{i-1}\right) \times\left(j_{i}-j_{i-1}\right)$-matrix with coefficients in $\mathbb{C}[z]$. The determinant $d_{i}(s)=\operatorname{det}\left(D_{i, i}(s)\right)$ is not identically zero, and we denote the degree of this determinant by $k_{i}(s)$.

These $k_{i}(s)$ are constrained only by the equations

$$
\begin{aligned}
c_{1} & =\sum_{s=1}^{m} k_{1}(s), \\
c_{2} & =\sum_{s=2}^{m}\left(k_{1}(s)+k_{2}(s)\right), \\
c_{3} & =\sum_{s=3}^{m}\left(k_{1}(s)+k_{2}(s)+k_{3}(s)\right), \\
& \vdots \\
c_{m} & =\sum_{j=1}^{m} k_{j}(m) .
\end{aligned}
$$

Here, of course, $C=\left(c_{1}, \cdots, c_{m}\right)$ is the total Chern degree of the map $f$.

Also, for convenience we define

$$
\begin{aligned}
& \mathscr{D}_{i}(s)=\left(\begin{array}{cccc}
D_{1,1}(s) & E_{1,2}(s) & \cdots & E_{1, i-1}(s) \\
0 & D_{2,2}(s) & \cdots & E_{2, i-1}(s) \\
\vdots & \vdots & \ddots & \vdots \\
0 & 0 & \cdots & D_{i-1, i-1}(s)
\end{array}\right), \\
& \mathscr{E}_{i}(s)=\left(\begin{array}{c}
E_{1, i}(s) \\
\vdots \\
E_{i-1, i}(s)
\end{array}\right),
\end{aligned}
$$

so $\mathscr{E}_{i}(s)$ is a $j_{i} \times\left(j_{i+1}-j_{i}\right)$-matrix with entries in $\mathbb{C}[z]$ and

$$
\mathscr{D}_{i+1}(s)=\left(\begin{array}{cc}
\mathscr{D}_{i}(s) & \mathscr{E}_{i}(s) \\
0 & D_{i, i}(s)
\end{array}\right)
$$

Throughout the remainder of the paper we will be assuming the $D_{i, i}(s)$ are in normal form, so the $k$ th column of $D_{i, i}(s), D_{i, i}^{k}(s)$, has the form 


$$
\left(\begin{array}{c}
f_{1}^{k}(s) \\
\vdots \\
f_{k}^{k}(s) \\
0 \\
\vdots \\
0
\end{array}\right)
$$

with $f_{k}^{k}(s)$ monic and $\operatorname{deg}\left(f_{r}^{k}(s)\right)<\operatorname{deg}\left(f_{k}^{k}(s)\right)$ for $r<k$.

Similarly, the $k$ th column of $\mathscr{E}_{i}(s)$,

$$
\mathscr{E}_{i}^{k}(s)=\left(\begin{array}{c}
e_{1}^{k}(s) \\
\vdots \\
e_{v}^{k}(s)
\end{array}\right)
$$

satisfies $\operatorname{deg}\left(e_{r}^{k}(s)\right)<\operatorname{deg}\left(f_{k}^{k}(s)\right)$ for all $r$. We also write

$$
N_{s}=\left(\begin{array}{l}
K_{s} \\
L_{s}
\end{array}\right) \text {, }
$$

where $L_{s}$ is $\left(j_{s}-j_{s-1}\right) \times\left(j_{s+1}-j_{s}\right)$ and $K_{s}$ is $j_{s-1} \times\left(j_{s+1}-j_{s}\right)$. Finally, we write

$$
\left[P_{s}, Q_{s}\right]=\left[D_{s}, N_{s}\right]\left[D_{s+1}, N_{s+1}\right] \cdots\left[D_{m}, N_{m}\right]
$$

as

$P_{s}=\left(\begin{array}{cccc}D_{1,1}(s) \cdots D_{1,1}(m) & H_{1,2}(s) & \cdots & H_{1, s}(s) \\ 0 & D_{2,2}(s) \cdots D_{2,2}(m) & \cdots & H_{2, s}(s) \\ \vdots & \vdots & \ddots & \vdots \\ 0 & 0 & \cdots & D_{s, s}(s) \cdots D_{s, s}(m)\end{array}\right)$

and

$$
Q_{s}=\left(\begin{array}{cccc}
J_{1, s+1}(s) & J_{1, s+2}(s) & \cdots & J_{1, m+1}(s) \\
J_{2, s+1}(s) & J_{2, s+2}(s) & \cdots & J_{2, m+1}(s) \\
\vdots & \vdots & \ddots & \vdots \\
J_{s, s+1}(s) & J_{s, s+2}(s) & \cdots & J_{s, m+1}(s)
\end{array}\right)
$$

Here we introduce the further notation

$$
\mathscr{H}_{i}(s)=\left(\begin{array}{c}
H_{1, i}(s) \\
\vdots \\
H_{i-1, i}(s)
\end{array}\right)
$$


for $2 \leq i \leq s$ are the off-diagonal block matrix columns appearing in (3.17) and

$$
\mathscr{J}_{j}(s)=\left(\begin{array}{c}
J_{1, j}(s) \\
\vdots \\
J_{s, j}(s)
\end{array}\right)
$$

for $s+1 \leq j \leq m+1$ are the block matrix columns appearing in (3.18).

Notice that when $s=m$

$$
\mathscr{J}_{m+1}(m)=N_{m}
$$

When $s<m$ direct expansion of the first block matrix column of (3.18), using (3.15) and (3.16), gives the following key equation:

$$
\mathscr{J}_{s+1}(s)=N_{s} D_{s+1, s+1}(s+1) \cdots D_{s+1, s+1}(m)+\sum_{j=0}^{m-s-1} \mathscr{T}(j)
$$

where

$$
\begin{aligned}
\mathscr{T}(j)=\mathscr{D}_{s+1}(s) \cdots \mathscr{D}_{s+1}(s+j) \mathscr{E}_{s+2}(s+j+1) \\
\cdot D_{s+1, s+1}(s+j+2) \cdots D_{s+1, s+1}(m) .
\end{aligned}
$$

There are analogous expansions for the $\mathscr{H}_{i}(s)$ and $\mathscr{J}_{j}(s)$ when $j \neq s+1$ but (3.19) is the key equation for the following reason. The fact that (3.11) represents the based map given in (3.12) implies, among other conditions, that $\mathscr{J}_{s+1}(s)$ must satisfy the constraint condition $\lim _{z \rightarrow \infty}\left(P_{s}^{-1} \mathscr{J}_{s+1}(s)\right)=$ 0 . In the next section we shall see that this is the fundamental constraint in that all other basing constraints follow from this one (see Lemma 4.6). Recall from [11, §3] that the constraints for a single $N$ matrix in the Grassmannian case came directly from evaluating $D^{-1} N$ as $z \rightarrow \infty$. The computation in the general case requires that we make this same computation for each $P_{s}^{-1} Q_{s}$ for $1 \leq s \leq m$ and the answer depends on the degree pattern of the associated multipartition for the $D_{s}$ 's as given in (3.14).

For example, the elements in $\operatorname{Rat}_{(1,1)}(\mathbb{F}(1,2,5))$ occur in two ways, first with $\left[P_{2}, Q_{2}\right]$ having pattern

$$
\left[\left(\begin{array}{cc}
1 & a \\
0 & z-b
\end{array}\right),\left(\begin{array}{ccc}
0 & 0 & 0 \\
\alpha_{1} & \alpha_{2} & \alpha_{3}
\end{array}\right)\right]
$$

so $\left[D_{1}(z), N_{1}(z)\right]$ must have the form $[z-c,-a]$. Alternately, $\left[P_{2}, Q_{2}\right]$ can have the pattern

$$
\left[\left(\begin{array}{cc}
z-1 & 0 \\
0 & 1
\end{array}\right),\left(\begin{array}{ccc}
\beta_{1} & \beta_{2} & \beta_{3} \\
0 & 0 & 0
\end{array}\right)\right]
$$

in which case $\left[D_{1}(z), N_{1}(z)\right]$ must have the form $[1, \gamma]$. 
The first case corresponds to the pattern of multidegrees, $k_{1}(1)=1$, $k_{1}(2)=0, k_{2}(2)=1$, and has complex dimension 6 . The second has $k_{1}(1)=0, k_{1}(2)=1, k_{2}(2)=0$ and has complex dimension 5 . It will be helpful to keep these simple examples in mind when we discuss the general case in the next section.

\section{Normal forms for flag manifolds: the basing condition}

We now discuss how the basing conditions determine the permissible forms in order that equation (3.11) represent an element of $\operatorname{Rat}_{C}(\mathbb{F}(J))$. We begin by considering the pair

$$
\begin{aligned}
\left(p_{i}(f), p_{i+1}(f)\right) & =\left(\left[\left(D_{i}, N_{i}\right) P_{i+1},\left(D_{i}, N_{i}\right) Q_{i+1}\right],\left[P_{i+1}, Q_{i+1}\right]\right) \\
& =\left(\left[P_{i}, Q_{i}\right],\left[P_{i+1}, Q_{i+1}\right]\right),
\end{aligned}
$$

where we have fixed a degree pattern on the diagonal determinants of the $D$ matrices (3.14). For simplicity write $D_{i}=A, N_{i}=B$,

$$
P_{i+1}=\left(\begin{array}{cc}
C & E \\
0 & F
\end{array}\right) \text { and } Q_{i+1}=\left(\begin{array}{c}
G \\
H
\end{array}\right)
$$

to get rid of subscripts. Here $C$ is $j_{i} \times j_{i}, E$ is $j_{i} \times\left(j_{i+1}-j_{i}\right), F$ is $\left(j_{i+1}-j_{i}\right) \times\left(j_{i+1}-j_{i}\right), G$ is $j_{i} \times\left(n-j_{i+1}\right)$ and $H$ is $\left(j_{i+1}-j_{i}\right) \times\left(n-j_{i+1}\right)$. Furthermore, we write $\kappa_{1}(i)=\operatorname{deg}(\operatorname{det}(A)), \kappa_{1}(i+1)=\operatorname{deg}(\operatorname{det}(C))$, and $\kappa_{2}(i+1)=\operatorname{deg}(\operatorname{det}(F))$. Notice that $c_{i}=\kappa_{1}(i)+\kappa_{1}(i+1)$ and $c_{i+1}=\kappa_{1}(i+1)+\kappa_{2}(i+1)$. Then

$$
P_{i+1}^{-1}=\left(\begin{array}{cc}
C^{-1} & -C^{-1} E F^{-1} \\
0 & F^{-1}
\end{array}\right)
$$

so the original basing conditions on $p_{i+1}(f)=\left[P_{i+1}, Q_{i+1}\right]$ are given by

$$
\begin{gathered}
\lim _{z \rightarrow \infty} C^{-1}\left(G-E F^{-1} H\right)=0, \\
\lim _{z \rightarrow \infty} F^{-1} H=0 .
\end{gathered}
$$

The $j_{i} \times n$ plane determined by $\left[P_{i}, Q_{i}\right]$ is

$$
[A C, A E+B F, A G+B H],
$$

so the next basing conditions are

$$
\begin{aligned}
& \lim _{z \rightarrow \infty} C^{-1}\left(E+A^{-1} B F\right)=0, \\
& \lim _{z \rightarrow \infty} C^{-1}\left(G+A^{-1} B H\right)=0 .
\end{aligned}
$$


Lemma 4.6. The basing condition in (4.5) is redundant. More exactly, (4.2), (4.3) and (4.4) imply (4.5).

Proof. We are interested in the behavior of these equations as $z$ goes to infinity. Since both (4.4) and (4.3) go to zero as $z \rightarrow \infty$, their product

$$
C^{-1}\left(E+A^{-1} B F\right) F^{-1} H
$$

must also tend to zero as $z \rightarrow \infty$. But adding this equation to (4.2) yields (4.5). q.e.d.

Thus, given the block diagonal entries of the two $D$ matrices in (4.1), namely $A, C$, and $F$, we can use (4.2), (4.3), and (4.4) to determine, in order, the possible entries in the following:

1. $H$ from (4.3).

2. The additional constraints imposed on $E$ and $B$ from (4.4). As we shall see below it is best to view these constraints as symmetric in $E$ and $B$ rather than fixing one to determine the other.

3. $G$ from (4.2) and condition 2 above, or equivalently (by Lemma 4.6) from (4.5) and condition 2 .

We now want to determine the precise deviation from the basing condition in

$$
\operatorname{Rat}_{\kappa_{1}(i)}\left(\mathbb{G}_{j_{i}, j_{i+1}-j_{i}}\right)
$$

that arises in the pair $[A, B]$. Write $A^{-1} B$ as the sum of a polynomial and a fractional part,

$$
A^{-1} B=V+W, \quad \text { with } V \in M_{j_{i}, j_{i+1}-j_{i}}(\mathbb{C}[z]), \lim _{z \rightarrow \infty}(W)=0 .
$$

It follows that $B=A V+A W=M+R$ where both $M, R$ are polynomial, but

$$
\lim _{z \rightarrow \infty}\left(A^{-1} R\right)=0 .
$$

Lemma 4.7. $[A, R] \in \operatorname{Rat}_{k_{1}}\left(\mathbb{G}_{j_{i}, j_{i+1}-j_{i}}\right)$.

Proof. From the construction of $R$ it follows that $[A, R]$ is based. It remains to verify that $A$ and $R$ are coprime. But $A$ and $B$ are coprime so there are $K, L$ with $A K+B L=I$. Hence, $A K+(A V+R) L=$ $A(K+V L)+R L=I$ and Lemma 4.7 follows. q.e.d.

It remains to see exactly how the solutions to $C^{-1}\left(E+A^{-1} B F\right)$ are constructed and what degrees of freedom we have. For the moment we assume $C, A$, and the term $R$ in $B$ are fixed, so $B$ varies by $A V$. It is not hard to show that $\lim _{z \rightarrow \infty}\left(C^{-1} V\right)=0$ though this is not strictly necessary to our results. The following set of examples are instructive. 
Example 4.8. Let

$$
\begin{aligned}
& A=\left(\begin{array}{cc}
1 & z+1 \\
0 & z^{2}-2
\end{array}\right) \text { so } A^{-1}=\left(\begin{array}{cc}
1 & \frac{-(z+1)}{z^{2}-2} \\
0 & \frac{1}{z^{2}-2}
\end{array}\right), \\
& C=\left(\begin{array}{cc}
z^{2} & 3 \\
0 & z-1
\end{array}\right) \text { so } C^{-1}=\left(\begin{array}{cc}
\frac{1}{z^{2}} & \frac{-3}{z^{2}(z-1)} \\
0 & \frac{1}{z-1}
\end{array}\right) .
\end{aligned}
$$

Let $P$ be a column vector with entries in $\mathbb{C}[z]$. Then

$$
\begin{aligned}
& \lim _{z \rightarrow \infty}\left(C^{-1} P\right)=0 \quad \text { if and only if } P=P_{1} z+P_{0}, \text { where } P_{1}=\left(\begin{array}{c}
p_{11} \\
0
\end{array}\right), \\
& \lim _{z \rightarrow \infty}\left(A^{-1} P\right)=0 \quad \text { if and only if } P=\left(\begin{array}{c}
p \\
p z+q
\end{array}\right) .
\end{aligned}
$$

Consequently,

$$
B=A W+A V=\left(\begin{array}{c}
p \\
p z+q
\end{array}\right)+A\left(\begin{array}{c}
p^{\prime} z+p^{\prime \prime} \\
q^{\prime}
\end{array}\right)
$$

with $q \neq \pm \sqrt{2 p}$ in order to assure that $[A, R] \in \operatorname{Rat}_{2}\left(\mathbb{G}_{2,1}\right)$.

Let $F=f_{11}=z-2$ so $E=\left(\begin{array}{l}e_{1} \\ e_{2}\end{array}\right)$ is a constant matrix. (We are assuming throughout that $\left[P_{i+1}, Q_{i+1}\right]$ is in normal form.) Then a short calculation shows that

$$
E+A^{-1} B F=\left(\begin{array}{c}
p^{\prime} \\
0
\end{array}\right) z^{2}+\left(\begin{array}{c}
p^{\prime \prime}-2 p^{\prime} \\
q^{\prime}
\end{array}\right) z+\left(\begin{array}{c}
e_{1}-p-q-2 p^{\prime \prime} \\
e_{2}+p-2 q^{\prime}
\end{array}\right)+L_{-1},
$$

where $L_{-1}$ has entries which are rational functions of $z$ that go to zero as $z \rightarrow \infty$. Consequently, in order for the basing condition (4.4) to be satisfied, (4.9) implies that $p^{\prime}=q^{\prime}=0$ while $e_{1}, e_{2}$, and $p^{\prime \prime}$ are arbitrary. It follows that, in this case,

$$
V=\left(\begin{array}{c}
p^{\prime \prime} \\
0
\end{array}\right), \quad E=\left(\begin{array}{l}
e_{1} \\
e_{2}
\end{array}\right)
$$

and $E, V$ together have three degrees of freedom. Notice that this is precisely the total amount of freedom one has in choosing a matrix $T \in$ $M_{2,1}(\mathbb{C}[z])$ which satisfies $\lim _{z \rightarrow \infty}\left(C^{-1} T\right)=0$.

Example 4.10. Next, suppose $A$ and $C$ are as given in Example 4.8, but now $F=f_{1,1}=z^{5}-2$. In this case we can write

$$
E=E_{4} z^{4}+E_{3} z^{3}+E_{2} z^{2}+E_{1} z+E_{0},
$$


and

$$
A^{-1} R f_{1,1}=L_{4} z^{4}+L_{3} z^{3}+L_{2} z^{2}+L_{1} z+L_{-1}
$$

while

$$
V f_{1,1}=\left(\begin{array}{c}
p^{\prime} \\
0
\end{array}\right) z^{6}+\left(\begin{array}{c}
p^{\prime \prime} \\
q^{\prime}
\end{array}\right) z^{5}-2\left(\begin{array}{c}
p^{\prime} \\
0
\end{array}\right) z-2\left(\begin{array}{c}
p^{\prime \prime} \\
q^{\prime}
\end{array}\right) .
$$

Since $E_{5}=E_{6}=0$ from the normal form constraints in $\left[P_{2}, Q_{2}\right]$, it follows that $V \equiv 0$ and

$$
\begin{aligned}
& E_{4}+L_{4}=0, \\
& E_{3}+L_{3}=0, \\
& E_{2}+L_{2}=0, \\
& E_{1}+L_{1}=\left(\begin{array}{l}
p \\
0
\end{array}\right), \\
& E_{0}+L_{0}=\left(\begin{array}{l}
p^{\prime} \\
q^{\prime}
\end{array}\right) .
\end{aligned}
$$

Once more there are three degrees of freedom in the solution, but here $V \equiv 0$ and all the degrees of freedom are concentrated in $E$.

In one sense Examples 4.8 and 4.10 are too special. The three basic solution vectors are either in $E$ or in $V$, and this would lead us to expect that $\operatorname{Rat}_{C}(\mathbb{F}(J))$ might be a union of elements described simply by fixing $A, R, C$, and $F$, and then letting $E$ and $V$ run independently through a direct sum decomposition of the appropriate dimension. But this is not true. It is easy to construct a $C$ and $f_{1,1}$ so the set of solution pairs $(E, V)$ to $\lim _{z \rightarrow \infty}\left(C^{-1}\left(E+V f_{1,1}\right)\right)=0$ do not split as a direct sum.

Example 4.11. Let $f_{11}=z^{2}+z+2$, and

$$
C=\left(\begin{array}{cc}
z & z^{3}+2 \\
0 & z^{4}-1
\end{array}\right) \text {. }
$$

Then

$$
V=\left(\begin{array}{c}
v_{3} z^{2}+v_{2} z+u_{0} \\
v_{3} z^{3}+v_{2} z^{2}+v_{1} z^{1}+v_{0}
\end{array}\right)
$$

but for $C^{-1}\left(E+V f_{1,1}\right)$ to go to zero as $z \rightarrow \infty$ we must have $v_{3}=v_{2}=0$ and $v_{1}=u_{0}$. But then it follows that $v_{0}=e_{11}$ where

$$
E=\left(\begin{array}{l}
e_{11} z+e_{12} \\
e_{21} z+e_{22}
\end{array}\right) \text {. }
$$

However, it is routine to check that $E$ and $V$ still span a 5-dimensional solution space and again this is precisely the dimensional freedom one has in selecting a matrix $T$ such that $\lim _{z \rightarrow \infty}\left(C^{-1} T\right)=0$. 
Hence, the important thing which all three of the examples above do show is that, in general, one must use both $E$ and $V$ in order to obtain a sufficiently large solution space to construct all elements which satisfy both the basing conditions and a given degree pattern. We now show that for any $A, C$, and $F$, the solution space always has dimension (over C) exactly equal to $\left(j_{i+1}-j_{i}\right) \operatorname{deg}(\operatorname{det}(C))$. To do so we first require the following elementary observation.

Lemma 4.12. Let $\mathscr{V}_{c} \subset(\mathbb{C}[z])^{n}$ be the set of all $n$-tuples

$$
\left(\begin{array}{c}
v_{1}(z) \\
\vdots \\
v_{n}(z)
\end{array}\right)
$$

with $\operatorname{deg}\left(v_{i}(z)\right)<c$ for all $i$. Let $f \in \mathbb{C}[z]$ be any monic polynomial of degree $c$, and let

$$
\mathscr{W}_{f}=\operatorname{im}\left\{f \circ:(\mathbb{C}[z])^{n} \rightarrow(\mathbb{C}[z])^{n}\right\}
$$

be the image of the map

$$
\left(\begin{array}{c}
v_{1} \\
\vdots \\
v_{n}
\end{array}\right) \mapsto\left(\begin{array}{c}
f v_{1} \\
\vdots \\
f v_{n}
\end{array}\right)
$$

Then $\mathscr{V}_{c}+\mathscr{W}_{f}=(\mathbb{C}[z])^{n}$. Indeed, $\mathscr{V}_{c} \cap \mathscr{W}_{f}=0$ so there is a direct sum decomposition

$$
\mathscr{V}_{c} \oplus \mathscr{W}_{f}=(\mathbb{C}[z])^{n}
$$

Proof. It suffices to observe that Lemma 4.12 is true when $n=1$, but there it is just the division algorithm in $\mathbb{C}[z]$. q.e.d.

Theorem 4.13. If we fix $A, R, C$, and $F$, then the set of $(E, V)$ which satisfy the basing condition at infinity has exactly $\left(j_{i+1}-j_{i}\right) \times \kappa_{1}(i+1)$ degrees of freedom.

Proof. Let $\mathscr{V}_{C} \subset(\mathbb{C}[z])^{j_{i}}$ be the subspace of all vectors $\mu$ in $(\mathbb{C}[z])^{j_{i}}$ with the property that $\lim _{z \rightarrow \infty}\left(C^{-1} \mu\right)=0$. Note $\operatorname{dim}_{\mathbb{C}} \mathscr{V}_{C}=\operatorname{deg}(\operatorname{det}(C))$. If $\mu \in V_{C}$ and $f \in \mathbb{C}[z]$ is any monic polynomial of degree $r$, then, by Lemma 4.12, we can write

$$
\mu=\theta+f \circ \omega, \quad \theta \in \mathscr{V}_{r}, \omega \in(\mathbb{C}[z])^{j_{i}} .
$$

Next write both $F=\left(F^{1}, F^{2}, \cdots, F^{j_{i+1}-j_{i}}\right)$ and $E=\left(E^{1}, \cdots, E^{j_{i+1}-j_{i}}\right)$ as a set of column vectors.

The normal form constraints imply that $E^{l} \in \mathscr{T}_{\operatorname{deg}\left(f_{l, l}\right)}$ for each $l$. Moreover, the same is true for the polynomial part of $A^{-1} R F$, which we 
denote by $P\left(A^{-1} R F\right)$; that is, the $l$ th column $P\left(A^{-1} R F\right)^{l} \in \mathscr{V}_{\operatorname{deg}\left(f_{l, l}\right)}$ for all $l$. Also we may write

$$
V F^{l}=V^{1} f_{1, l}+V^{2} f_{2, l}+\cdots+V^{l} f_{l, l} .
$$

Assume for the moment that there exists a solution $E_{0}$ and $V_{0}$. Then a direct computation shows that

$$
\theta_{0}^{l}=V_{0}^{l} f_{l, l}+E_{0}^{l}+P\left(A^{-1} R F\right)^{l}+\sum_{m=1}^{l-1} V_{0}^{m} f_{m, l}
$$

is an element of $\mathscr{V}_{C}$. But Lemma 4.12 implies that for every $\mu_{l} \in \mathscr{V}_{C}$ we can inductively solve for $V^{l}$ and $E^{l}$ so that $\theta_{0}^{l}+\mu_{l} \in \mathscr{V}_{C}$ can be written as

$$
V^{l} f_{l, l}+E^{l}+P\left(A^{-1} R F\right)^{l}+\sum_{m=1}^{l-1} V^{m} f_{m, l}=\theta_{0}^{l}+\mu_{l}
$$

with $E^{l} \in \mathscr{V}_{\operatorname{deg}\left(f_{l, l}\right)}$. Hence, the existence of one fixed solution implies that each of the $j_{i+1}-j_{i}$ columns contributes exactly $\operatorname{deg}(\operatorname{det}(C))$ dimensions to the solution space.

To complete the proof it suffices to show that the solution space is not empty. For this first notice that if $\kappa_{1}(i+1)=0$, i.e., $C$ is the identity matrix, then $E=P\left(A^{-1} R F\right)$ and $V=0$ is the unique solution to (4.14) for any given $A, R=B$, and $F$. Thus, the theorem is true in the case when $C=I$ (which we will see later is actually the generic situation in $\left.\operatorname{Rat}_{C}(\mathbb{F}(J))\right)$. Hence, we can assume that $\kappa_{1}(i+1)>0$. But whenever a diagonal entry of $C, c_{l l}$, has positive degree, the vector

$$
\left(\begin{array}{c}
0 \\
\vdots \\
1 \\
0 \\
\vdots \\
0
\end{array}\right)
$$

is an element of $V_{C}$. Here the entry 1 occurs in the $l$ th row. Now use these elements of $V_{C}$ to inductively construct a solution to equation (4.14). q.e.d.

Corollary 4.17. If we fix $A, C$, and $F$, then the set of $(R, E, V)$ (equivalently the set of $(B, E)$ ) which satisfy the basing condition at infinity has exactly $\operatorname{deg}(\operatorname{det}(A C))\left(j_{i+1}-j_{i}\right)$ degrees of freedom. 
Proof. Theorem 4.13 implies that for each fixed $R$ there are exactly $\operatorname{deg}(\operatorname{det}(C))\left(j_{i+1}-j_{i}\right)$ degrees of freedom while the set of admissible $R$ contributes exactly $\operatorname{deg}(\operatorname{det}(A))\left(j_{i+1}-j_{i}\right)$ degrees of freedom.

Corollary 4.18. The complex dimension of the space of all elements of the form

$$
[A, B],\left[\left(\begin{array}{cc}
C & E \\
0 & F
\end{array}\right),\left(\begin{array}{l}
G \\
H
\end{array}\right)\right] \in \operatorname{Rat}_{c_{i}, c_{i+1}}\left(\mathbb{F}\left(j_{i}, j_{i+1}\right)\right)
$$

is $c_{i}\left(j_{i+1}-j_{i}\right)+c_{i+1}\left(n-j_{i+1}\right)+k_{1}(i)\left(j_{i}\right)+k_{1}(i+1)\left(j_{i}\right)+k_{2}(i+1)\left(j_{i+1}-j_{i}\right)$.

Proof. First fix $A, C$, and $F$. The $c_{i}\left(j_{i+1}-j_{i}\right)$ term comes from the freedom to vary $(B, E)$ as in Corollary 4.17 while the $c_{i+1}\left(n-j_{i+1}\right)$ term comes from the freedom to vary $G$ and $H$ which is determined by the conditions (4.2) and (4.3). Finally, the last three terms in the sum come from the freedom to vary $A, C$, and $F$ as shown in the proof of Corollary 4.21 (a) below. q.e.d.

We conclude this section by analyzing the general case. Although this is notationally formidable all the mathematical ideas have already been presented in the proof of Theorem 4.13. At this point the reader is advised to review equations (3.13) through (3.20) to recall the block matrix pieces that replace the $A, B, C, E, F, G$, and $H$ that appeared in the analysis above.

In fact, recalling (3.19) and (3.20), we have the direct generalization of Theorem 4.13.

Theorem 4.19. Fix the $D_{i, i}(s)$ matrices for $s$ fixed and $1 \leq i \leq m$. Then the set of solutions $\mathscr{J}_{s+1}(s)$ to the fundamental basing constraint equation

$$
\lim _{z \rightarrow \infty}\left(P_{s}^{-1} \mathscr{J}_{s+1}(s)\right)=0
$$

has dimension $\operatorname{deg}\left(\operatorname{det}\left(P_{s}\right)\right) \cdot\left(j_{s+1}-j_{s}\right)$ as a vector space over $\mathbb{C}$.

Proof. The proof is a formal extension of Theorem 4.13 and is perhaps best understood by explicitly working out the situation for $m=3$ and $\mathscr{J}_{2}(1)$. Here the constraint equation for $\mathscr{J}_{2}(1)$ expanded out is

$$
\begin{aligned}
& D_{1,1}^{-1}(3)\left[E_{1,2}(3)+D_{1,1}^{-1}(s)\left[E_{1,2}(2)+D_{1,1}^{-1}(1) N_{1} D_{2,2}(2)\right] D_{2,2}(3)\right] \\
& \quad=P_{1}^{-1} \mathscr{J}_{2}(1) .
\end{aligned}
$$

We now just iterate Theorem 4.13 on the individual terms working from the inside brackets out. We can write $N_{1}=R_{1}+D_{1,1}(1) V_{1}$ where $D_{1,1}^{-1}(1) R_{1} \rightarrow 0$ as $z \rightarrow \infty$. As we observed above, the polynomial part of $D_{1,1}^{-1}(1) R_{1} D_{2,2}$ (2) lies in the vector space of possible $E_{1,2}(2)$ 's so we are 
free to choose $R_{1}$ subject only to the constraint that $\lim _{z \rightarrow \infty}\left(D_{1,1}^{-1}(1) R_{1}\right)=$ 0 . This first choice gives us $\operatorname{deg}\left(\operatorname{det}\left(D_{1,1}(1)\right)\right)\left(j_{2}-j_{1}\right)$ degrees of freedom in the solution space by Theorem 4.13. The resulting freedom of choice for the next piece, $\left(E_{1,2}(2), V_{1}\right)$, gives any arbitrary vector as the polynomial part of

$$
E_{1,2}(2)+D_{1,1}^{-1}(1) N_{1} D_{2,2}(2)=R_{2}+D_{1,1}(2) V_{2} \text {. }
$$

Again, $\lim _{z \rightarrow \infty}\left(D_{1,1}^{-1}(2) R_{2}\right)=0$ so Theorem 4.13 shows this piece contributes $\operatorname{deg}\left(\operatorname{det}\left(D_{1,1}(2)\right)\right)\left(j_{2}-j_{1}\right)$ more dimensions to the solution space.

Consequently, since $D_{1,1}^{-1}(2) R_{2} D_{2,2}(3)$ and $D_{1,1}^{-1}(2) W D_{2,2}(3)$ lie in the space of possible $E_{1,2}(3)$ 's for $W$ satisfying $\lim _{z \rightarrow \infty}(W)=0$, we see that Theorem 4.13 implies the next pair $\left(E_{1,2}(3), V_{2}\right)$ is free to determine any polynomial vector satisfying our constraints, $R_{3}=E_{1,2}(3)+V_{2} D_{2,2}(3)$ (with freedom of choice in $R_{1}$ and $R_{2}$ ). But $D_{1,1}^{-1}(3) R_{3} \rightarrow 0$ as $z \rightarrow \infty$, so Theorem 4.13 shows that the dimension of the space of such $R_{3}$ 's is equal to $\operatorname{deg}\left(\operatorname{det}\left(D_{1,1}(3)\right)\right)\left(j_{2}-j_{1}\right)$. Thus, the total amount of freedom we have at this point is exactly

$$
\left(\sum_{1}^{3} \operatorname{deg}\left(\operatorname{det}\left(D_{1,1}(i)\right)\right)\right)\left(j_{2}-j_{1}\right)=\operatorname{deg}\left(\operatorname{det}\left(P_{1}\right)\right)\left(j_{2}-j_{1}\right) .
$$

The general case now follows by double induction on $s$ and $m$. q.e.d.

Finally, we have the generalizations of Corollaries 4.17 and 4.18.

Corollary 4.20. Fix the $D_{i, i}(s)$ for all $i$ and $s$. Then the complex dimension of the space of all elements of the form

$$
\left[D_{1}, N_{1}\right]\left[D_{2}, N_{2}\right] \cdots\left[D_{m}, N_{m}\right] \in \operatorname{Rat}_{C}(\mathbb{F}(J))
$$

with these fuxed $D_{i, i}(s)$ is $\sum_{1}^{m} c_{i}\left(j_{i+1}-j_{i}\right)$ where $j_{m+1}=n$.

Proof. When each of the $D_{i, i}(s)$ 's is fixed, the degrees of freedom are completely described by $\mathscr{J}_{s+1}(s)$ for $1 \leq s \leq m$. q.e.d.

Corollary 4.21. Fix the degree pattern $\left\{k_{i}(j), i \leq j\right\}$ satisfying (3.14) for a given $C=\left(c_{1}, \cdots, c_{m}\right)$.

(a) The complex dimension of the subspace of $\operatorname{Rat}_{C}(\mathbb{F}(J))$ consisting of all elements of the form $\left[D_{1}, N_{1}\right] \cdots\left[D_{m}, N_{m}\right]$ with the multidegrees of the $D_{j}$ 's equal to the $k_{i}(j)$ 's is $\sum_{1}^{m} c_{i}\left(j_{i+1}-j_{i}\right)+\sum_{i, s} k_{i}(s)\left(j_{i}-j_{i-1}\right)$.

(b) The complex dimension of $\operatorname{Rat}_{C}(\mathbb{F}(J))$ is $\sum c_{i}\left(j_{i+1}-j_{i-1}\right)$ and there is one and only one pattern of multidegrees for which this dimension is achieved, namely, $k_{i}(i)=c_{i}, k_{i}(j)=0$ for $j>i$. Here $j_{0}=0$.

Proof. The first summand term comes directly from Corollary 4.20. The second summand counts the degrees of freedom contributed by each 
$D_{i, i}(s)$. The greatest number of degrees of freedom for an $r \times r$ matrix $T$, which is in normal form, with coefficients in $\mathbb{C}[z]$, where $\operatorname{deg}(\operatorname{det}(T))=$ $v$, occurs when

$$
T=\left(\begin{array}{ccccc}
1 & 0 & \cdots & 0 & f_{1} \\
0 & 1 & \cdots & 0 & f_{2} \\
\vdots & \vdots & \ddots & \vdots & \vdots \\
0 & 0 & \cdots & 1 & f_{r-1} \\
0 & 0 & \cdots & 0 & g_{r}
\end{array}\right)
$$

with $\operatorname{deg}\left(f_{i}\right)<\operatorname{deg}\left(g_{r}\right)$. Since the coefficients in each $f_{i}$ are arbitrary and $g_{r}$ is monic, there is freedom of choice for precisely $v r$ coordinates. From this Corollary 4.21(a) follows.

Next note that

$$
\left(\sum_{j} k_{i}(j)\right)\left(j_{i}-j_{i-1}\right) \leq c_{i}\left(j_{i}-j_{i-1}\right)
$$

from (3.14), and equality occurs if and only if all the $k_{r}(j)$ are zero for $j \geq i$ and $r \leq i$. Consequently, the only way in which equality can occur for all these sums is if $k_{i}(i)=c_{i}, k_{i}(j)=0$ for $j>i$. From this Corollary 4.21(b) follows.

\section{Stratification of $\operatorname{Rat}_{C}(\mathbb{F}(J))$ by normal forms}

We now use the normal form constructed in the two last sections in order to decompose $\operatorname{Rat}_{C}(\mathbb{F}(J))$ into smooth strata. Recall that the degrees of the determinants of the diagonal $D_{i, i}(s)$ blocks of the normal form partition the total Chern class $C=\left(c_{1}, \cdots, c_{m}\right)$ into "multipartitions"

$$
K=\left(k_{1}(1), k_{1}(2), k_{2}(2), \cdots, k_{1}(m), \cdots, k_{m}(m)\right),
$$

where the $C$ and $K$ are related by (3.14).

Definition 5.2. For each multipartition $K$ of $C$ let

$$
\begin{aligned}
Y(K) & =Y\left(k_{1}(1), \cdots, k_{m}(m)\right) \\
& =\left\{\begin{array}{l|l}
f \in \operatorname{Rat}_{C}(\mathbb{F}(J)) & \begin{array}{l}
\text { The normal form of } f \text { given in }(3.11) \\
\text { satisfies } \operatorname{deg}\left(\operatorname{det}\left(D_{i, i}(s)\right)\right)=k_{i}(s) .
\end{array}
\end{array}\right\} .
\end{aligned}
$$

Theorem 5.3. For each multipartition $K$ of $C, Y(K)$ is a complex submanifold of $\operatorname{Rat}_{C}(\mathbb{F}(J))$ of complex dimension as given in Corollary 4.21(a). 
Proof. The local submanifold coordinates for $Y(K)$ are determined by the free coefficients of the polynomials in the flag normal form described in $\S \S 3$ and 4. The fact that $Y(K)$ is a regularly embedded submanifold of $\operatorname{Rat}_{C}(\mathbb{F}(J))$ follows from the proof of Proposition 5.5 below. q.e.d.

Just as for the Grassmannian case, associated to the multipartitions of $C$, there is a filtration of $\operatorname{Rat}_{C}(\mathbb{F}(J))$ by open manifolds,

$$
\mathscr{F}_{K}\left[\operatorname{Rat}_{C}(\mathbb{F}(J))\right]=\bigcup_{K^{\prime} \geq K} Y\left(K^{\prime}\right),
$$

with natural inclusions

$$
i(K): X(K) \hookrightarrow \mathscr{F}_{K}\left[\operatorname{Rat}_{C}(\mathbb{F}(J))\right]
$$

Here we have imposed the natural lexicographic order (from the right) on the multipartitions (5.1) which explains the equation $K^{\prime} \geq K$.

Proposition 5.5. The image of $X(K)$ in $\mathscr{F}_{K}\left[\operatorname{Rat}_{C}(\mathbb{F}(J))\right]$ has a complex normal bundle $\nu(i(K))$.

Unlike the simpler Grassmannian case [11, $\S 4$ the normal bundles here are not, in general, trivial. Parts 1 and 2 of Theorem B follow from Corollary 4.21, Theorem 5.3 and Proposition 5.5. Before giving the proof of Proposition 5.5 it is worthwhile to consider some examples.

Example 5.6. We begin with the two strata of $\operatorname{Rat}_{(1,1)}(\mathbb{F}(1,2,5))$ (recall the examples given at the end of $\S 4$ ). The smallest stratum of $\operatorname{Rat}_{(1,1)}(\mathbb{F}(1,2,5))$ is given by elements of the form

$$
[1, \gamma], \quad\left[\left(\begin{array}{cc}
z-1 & 0 \\
0 & 1
\end{array}\right),\left(\begin{array}{ccc}
\beta_{1} & \beta_{2} & \beta_{3} \\
0 & 0 & 0
\end{array}\right)\right] .
$$

We perturb these elements by placing an $\varepsilon$ in the lower corner of the $D_{2}$ matrix to obtain

$$
[1, \gamma], \quad\left[\left(\begin{array}{cc}
z-1 & 0 \\
\varepsilon & 1
\end{array}\right),\left(\begin{array}{ccc}
\beta_{1} & \beta_{2} & \beta_{3} \\
0 & 0 & 0
\end{array}\right)\right]
$$

It is then a straightforward calculation that the following unimodular matrix

$$
U=\left(\begin{array}{cc}
0 & 1 / \varepsilon \\
-\varepsilon & z-1
\end{array}\right)
$$

can be used to rewrite (5.7) as

(5.8)

$$
\begin{aligned}
& {\left[D_{1}, N_{1}\right] U^{-1} U\left[D_{2}, N_{2}\right]} \\
& \quad=\left[z-1+\varepsilon \gamma, \frac{-1}{\varepsilon}\right], \quad\left[\left(\begin{array}{cc}
1 & 1 / \varepsilon \\
0 & z-1
\end{array}\right),\left(\begin{array}{ccc}
0 & 0 & 0 \\
-\varepsilon \beta_{1} & -\varepsilon \beta_{2} & -\varepsilon \beta_{3}
\end{array}\right)\right] .
\end{aligned}
$$


Thus, we see that an infinitesimal perturbation of an element in the 5dimensional strata can land in the 6-dimensional strata. Notice that rather than use the unimodular matrix $U$ to bring the perturbed matrix to normal form (5.8) that one can also verify directly that (5.7) still satisfies the rank condition and the base point condition, for both $p_{1}(f)$ and $p_{2}(f)$.

Example 5.9. Consider the element

$$
\left[\begin{array}{ccc}
z-1 & 0 & 1 \\
0 & 1 & 0
\end{array}\right], \quad\left[\begin{array}{ccccc}
z & 0 & 0 & \beta_{1} & \beta_{2} \\
0 & 1 & 0 & 0 & 0 \\
0 & 0 & 1 & 0 & 0
\end{array}\right]
$$

in $\operatorname{Rat}_{2,1}(\mathbb{F}(2,3,5))$. Let $L_{a, b}$ be the $3 \times 3$ matrix with a 1 in the $(a, b)$ th entry and zeros everywhere else. One can check that adding $L_{3,1}$ to the $D_{2}$ matrix in (5.10) perturbs the map to an element with $\operatorname{det}\left(D_{1,1}(1)\right)$ of degree two and new $D_{2}$ matrix of the form

$$
\left[\begin{array}{lll}
1 & 0 & 0 \\
0 & 1 & 0 \\
0 & 0 & z
\end{array}\right]
$$

However, there is another way to perturb (5.10) to this same element in two steps as follows. First, use the matrix $L_{2,1}$ to perturb the $D_{2}$ matrix in $(5.10)$ to the form

$$
\left[\begin{array}{lll}
1 & 0 & 0 \\
0 & z & 0 \\
0 & 0 & 1
\end{array}\right]
$$

This perturbation of the " $C$ " matrix does not change either the degree of $\operatorname{det}\left(D_{1,1}(1)\right)$ or the bottom row $[0, F, H]$ of $(5.10)$. If one thinks of the block rows of $\left[D_{2}, N_{2}\right]$ as representing a fibration of Grassmannian pieces and realizes that each Grassmannian piece is further stratified as described in $[11, \S 4]$ then this perturbation is recognized as a perturbation of the internal strata of the possible $C$ matrices of a fixed determinantal degree which does not affect the degrees of the determinants of either $A$ or $F$. One can next use $L_{3,2}$ to bring this new matrix to the original perturbed matrix described first which was obtained by using only $L_{3,1}$.

Remark 5.11. Example 5.9 points out that we must be careful in talking about "normal" directions to various strata. That is, one could further stratify each $Y(K)$ by the degrees of the individual diagonal entries of each of the $D_{i, i}(s)$ matrices, and the perturbations we describe below in the proof of Proposition 5.5 also describe the normal bundles of each strata in this finer stratification. As in the Grassmannian case but unlike 
Proposition 5.5, the normal bundles for each individual strata in this finer stratification are all trivial. For homotopy theoretic reasons we will need to consider a coarser filtration by complex submanifolds than the one defined in Definition 5.2 for $\operatorname{Rat}_{C}(\mathbb{F}(J))$. The proof we give below will also extend to show the normal bundles for the strata in this coarser filtration are also complex bundles.

Proof of Proposition 5.5. Let $f \in Y(K)$ be represented by the associated normal form

$$
\left(\left[D_{1}, N_{1}\right], \cdots,\left[D_{m}, N_{m}\right]\right)
$$

To compute the local coordinates for the normal bundle of $Y(K)$ we consider infinitesimal perturbations of the form

$$
f \mapsto f+\varepsilon S \in \operatorname{Rat}_{C}(\mathbb{F}(J))
$$

and analyze the first-order effect on the tangent level. Here $S=\left[0, S_{2}\right.$, $\cdots, S_{m}$ ] is to be regarded as $m-1$ matrix pairs which should be added term by term to (5.12). We may set $S_{1}=0$, for, as we stated in Remark 5.11, a permissible perturbation having $S_{1} \neq 0$ will split as a sum of two perturbations, the first tangent to $Y(K) \in \mathrm{Rat}_{C}(\mathbb{F}(J))$ and the second induced by a perturbation $\bar{S}$ where $\bar{S}_{1}=0$. Just as in the proof of Proposition 4.4 of [11], one must be careful when choosing $S$, in order not to leave the $C$ component of $\operatorname{Rat}(\mathbb{F}(J)$ ) (given by the region where, for all $r$, the $r \times r$ minors of $p_{r}(f)=\left[P_{r}, Q_{r}\right]$ all have degree $<c_{r}$ except for the minor $P_{r}$ itself). Using induction and the arguments from the proof of $[11,4.4]$ we shall see that the theorem follows from the fact that one can vary the coordinates of each $P_{r}$ (independently for each $r$ ) by small polynomials in the lower triangular regions, always taking care to keep the multidegrees sufficiently under control so that $p_{r}(f)+\varepsilon S$ remains in the $C$ component, and, having done that, checking that all the coordinates in the new $Q_{i}$ 's are uniquely determined, modulo the tangent direction to $Y(K)$, by this procedure.

Formally, we proceed by induction on $m$. When $m=1[11, \S 4]$ shows the normal bundles are actually trivial and hence complex. Next we consider the case $m=2$ and, using the notation of $\S 4$, write

$$
[A, B]\left[\left(\begin{array}{cc}
C & E \\
\varepsilon L & F
\end{array}\right),\left(\begin{array}{c}
G+\varepsilon G^{\prime} \\
H+\varepsilon H^{\prime}
\end{array}\right)\right],
$$

where

1. $L$ is any $j_{i} \times j_{i+1}-j_{i}$ matrix with $\operatorname{deg}\left(l_{\alpha, \beta}\right)<\operatorname{deg}\left(c_{\beta, \beta}\right)$. 
2. $G^{\prime}=G+T_{1}(L)$ and $H^{\prime}=H+T_{2}(L)$ are the associated perturbed " $R$ " matrices of $[11,4.6]$ which ensures that the perturbation stays in the Rat space. More precisely, these matrices are required in the perturbation so that the basing condition is still satisfied. It is a routine calculation that given any $L$ matrix satisfying our degree pattern such $T_{1}(L)$ and $T_{2}(L)$ exist.

3. Since the maximal rank condition is an open condition it follows that, for all sufficiently small $\varepsilon,(5.13)$ represents an element of $\operatorname{Rat}_{C}(\mathbb{F}(J))$.

4. It follows exactly as in the proof of 4.4 of [11] that it is sufficient to consider only such $L$ 's as given in item 1 . This follows as any matrix $L$ with entries of higher degree can be shown to split on the tangent space level as an $L$ as in item 1 and a perturbation in the $Y(K)$ direction.

Thus, for all sufficiently small $\varepsilon,[11,4.4]$ implies that $p_{2}(f)$ is a based map and hence an element of $\operatorname{Rat}_{k_{1}(i+1)+k_{2}(i+1)}\left(\mathbb{G}_{j_{2}, n-j_{2}}\right)$. Therefore, to prove Proposition 5.5 in this case it suffices to check that

$$
p_{1}(f)=\left[A C+\varepsilon B L, A E+B F, A\left(G+\varepsilon G^{\prime}\right)+B\left(H+\varepsilon H^{\prime}\right)\right]
$$

is a based map. By Lemma 4.6, it suffices to check that

$$
(A C+\varepsilon B L)^{-1}(A E+B F) \rightarrow 0
$$

as $z \rightarrow \infty$. Note that the degree conditions on $L$ ensure, for sufficiently small $\varepsilon$, that $(A C+\varepsilon B L)^{-1}$ exists and can be computed by the standard convergent power series expansion. It is then a straightforward calculation, using (4.3), (4.4), and the fact that $C^{-1} L$ is bounded, to show that (5.14) is satisfied. This shows that there is an injection from the set of all such $L$ matrices to $\nu(i(Y(K)))$ but comparing dimensions (recall Example 5.9 and Remark 5.11) shows this injection must be an isomorphism. Thus, the coordinates of such $L$ give the desired local trivialization.

Next, consider the case when $m=3$, and explicitly write (5.12) as

$$
\left[D_{1,1}(1), R_{1,2}(1)+M_{1,2}(1)\right]\left[D_{2}, N_{2}\right]\left[D_{3}, N_{3}\right],
$$

where

$$
\left[D_{2}, N_{2}\right]=\left[\begin{array}{ccc}
D_{1,1}(2) & E_{1,2}(2) & R_{1,3}(2)+M_{1,3}(2) \\
0 & D_{2,2}(2) & R_{2,3}(2)+M_{2,3}(2)
\end{array}\right]
$$

and

$$
\left[D_{3}, N_{3}\right]=\left[\begin{array}{cccc}
D_{1,1}(3) & E_{1,2}(3) & E_{1,3}(3) & R_{1,4}(3) \\
0 & D_{2,2}(3) & E_{2,3}(3) & R_{2,4}(3) \\
0 & 0 & D_{3,3}(3) & R_{3,4}(3)
\end{array}\right]
$$


Here the $R$ and $M$ matrices are as in $\S 4$.

An argument similar to the one given above shows that if we perturb $\left[D_{3}, N_{3}\right]$ by a lower triangular

$$
L=\left(\begin{array}{ccc}
0 & 0 & 0 \\
L_{2,1}(3) & 0 & 0 \\
L_{3,1}(3) & L_{3,2}(3) & 0
\end{array}\right),
$$

then the new map $f^{\prime}$ is in $Y\left(K^{\prime}\right) \subset \operatorname{Rat}_{C}(\mathbb{F}(J))$, and is given in terms of normal coordinates by

$$
\begin{aligned}
{\left[\widehat{D}_{1}, \widehat{N}_{1}\right]\left[\widehat{D}_{2}, \widehat{N}_{2}\right]\left[\widehat{D}_{3}, \widehat{N}_{3}\right] } & =\left[D_{1}, N_{1}\right]\left[D_{2}, N_{2}\right] U_{3}^{-1} U_{3}\left[D_{3}+\varepsilon L, N_{3}+\varepsilon T\right] \\
& =\left[D_{1}, N_{1}\right]\left[D_{2}, N_{2}\right] U_{3}^{-1}\left[\widehat{D}_{3}, \widehat{N}_{3}\right] \\
& =\left[D_{1}, N_{1}\right]\left[\bar{D}_{2}, \bar{N}_{2}\right]\left[\widehat{D}_{3}, \widehat{N}_{3}\right] \\
& =\left[D_{1}, N_{1}\right] U_{2}^{-1} U_{2}\left[\bar{D}_{2}, \bar{N}_{2}\right]\left[\widehat{D}_{3}, \widehat{N}_{3}\right] \\
& =\left[D_{1}, N_{1}\right] U_{2}^{-1}\left[\widehat{D}_{2}, \widehat{N}_{2}\right]\left[\widehat{D}_{3}, \widehat{N}_{3}\right] \\
& =\left[\bar{D}_{1}, \bar{N}_{1}\right]\left[\widehat{D}_{2}, \widehat{N}_{2}\right]\left[\widehat{D}_{3}, \widehat{N}_{3}\right] \\
& =U_{1}\left[\bar{D}_{1}, \bar{N}_{1}\right]\left[\widehat{D}_{2}, \widehat{N}_{2}\right]\left[\widehat{D}_{3}, \widehat{N}_{3}\right]
\end{aligned}
$$

where each $U_{r}$ is a unimodular matrix which brings the new $\widehat{D}_{r}$ matrix to normal form (2.1).

Next consider the perturbation on the second term

$$
\left[D_{2}+L, N_{2}+T(L)\right]=\left[\begin{array}{ccc}
D_{1,1}(2) & E_{1,2}(2) & R_{1,3}(2)+M_{1,3}(2)+\varepsilon T_{1}(L) \\
\varepsilon L & D_{2,2}(2) & R_{2,3}(2)+M_{2,3}(2)+\varepsilon T_{2}(L)
\end{array}\right] .
$$

While it is clear that we may rewrite the effect of the perturbation to the left as in (5.15), we must check that the perturbed map in the third matrix remains based and in $\operatorname{Rat}_{C}(\mathbb{F}(J))$.

Lemma 5.17. There are choices $\bar{T}_{1}(L), \bar{T}_{2}(L)$, and $\bar{T}_{3}(L)$ such that the element

$$
\left[D_{2}+L, N_{2}+T(L)\right]\left[D_{3}, N_{3}+\bar{T}(L)\right]
$$

is an element of $\operatorname{Rat}_{C}(\mathbb{F}(J))$.

Proof. This is a straightforward computation using the original basing equations as above. Again the key observation is that the degree conditions on $L$ insure, for sufficiently small $\varepsilon$, that $\left(D_{2}+\varepsilon L\right)^{-1}$ exists and can be computed by the standard convergent power series expansion. One also needs to observe that $D_{1,1}(2)^{-1} L$ is bounded. Finally, the choices of the $T$ 's and $\bar{T}$ 's are simply the matrix equivalents of the " $R$ " matrices of [11, 4.6]. q.e.d. 
Lemma 5.18 provides the inductive step and hence, using Theorem 4.18 and Remark 4.19, one sees that the allowable perturbations

$$
S=\left(0, S_{2}, \cdots, S_{m}\right)=\left(0, L_{1}+T_{1}, \cdots, L_{m}+T_{m}\right)
$$

of $f=\left(p_{1}(f), \cdots, p_{m}(f)\right)$ where each $L$ is lower triangular with $\operatorname{deg}\left(l_{\alpha, \beta}\right)<\operatorname{deg}\left(d_{\beta, \beta}\right)$ are in one-to-one correspondence with the pairwise independent allowable perturbations of each $\left[D_{r}, N_{r}\right]$ pair. A simple dimension count using Corollary 4.21 (again recall Remark 5.11) shows these $L$ then give the desired local trivialization of the normal bundle $\nu(i(K))$. q.e.d.

Finally, an elementary calculation, using the Euclidean algorithm inductively in each of the $r$ factors, implies that the normal directions for $Y(k)$ in $\operatorname{Rat}_{C}(\mathbb{F}(J))$ lie in the union of the higher-dimensional strata $Y\left(K^{\prime}\right)$ where $K<K^{\prime}$ in our lexicographic order. This analysis and the fact that Rat $c_{c_{r}}\left(\mathbb{G}_{i_{r}, n_{r}}\right)$ is a connected complex manifold [4] imply

Corollary 5.19. ${ }^{2} \operatorname{Rat}_{C}(\mathbb{F}(J))$ is a connected complex manifold of complex dimension $\sum c_{i}\left(j_{i+1}-j_{i-1}\right)$.

\section{Filtering the individual strata via Grassmannians}

We now analyze the geometry of an individual stratum $Y(K)$ where $K$ is a fixed multipartition of $C$. The general procedure, which generalizes the analysis for a single Grassmannian [11], is to exhibit the stratum $Y(K)$ as an iterated fibration, where each of the iterated fibers is, in turn, an iterate fibration with each of the smaller bases and fibers homotopy equivalent to various copies of $\operatorname{Rat}_{k}\left(\mathbb{G}_{n, m}\right)$ for appropriate choices of $k$, $n$, and $m$. In order to obtain an inductive procedure for decomposing $Y(K)$ we systematically "peel" off the bottom block row of each $\left[D_{r}, N_{r}\right]$ by projecting onto the "based Grassmannian" part of this product.

Proposition 6.1. Let $K$ be a multipartition

$$
\left(k_{1}(1), k_{1}(2), k_{2}(2), \cdots, k_{m}(1), \cdots, k_{m}(m)\right) .
$$

Then there is a sequence of fibrations

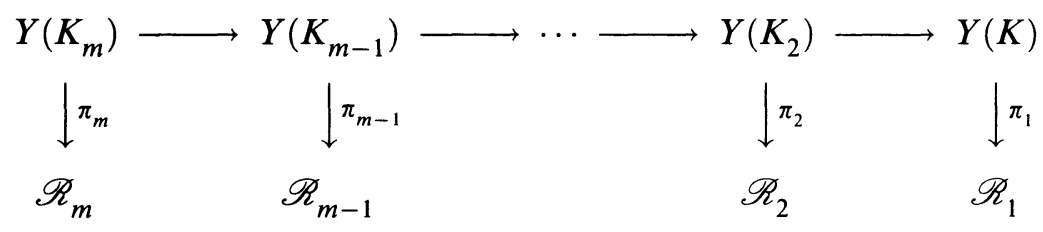


where each $\mathscr{R}_{r}$ is itself an iterate fibration

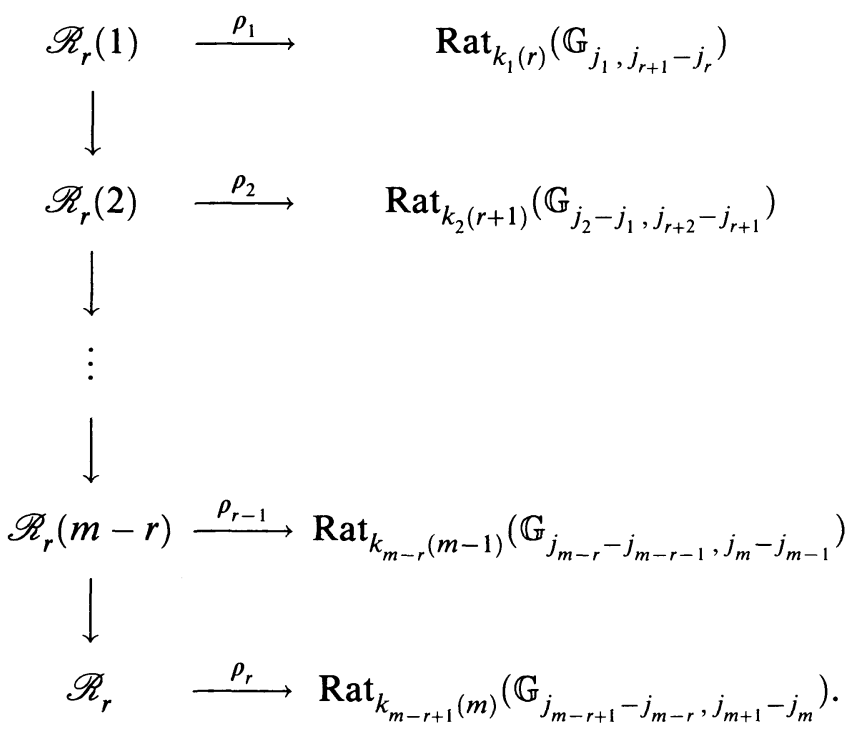

Here

(a) $\mathscr{R}_{r}$ is homotopy equivalent to the stratum

$$
Y\left(L_{r}\right)=Y\left(l_{1}(1, r), l_{1}(2, r), l_{2}(2, r), \cdots, l_{1}(m, r), \cdots, l_{m}(m, r)\right),
$$

where

$$
l_{a}(b, r)= \begin{cases}k_{a}(a+r-1) & \text { if } a=b, \\ 0 & \text { otherwise }\end{cases}
$$

(b) $\pi_{r}$ is a "peeling projection" defined below in terms of normal coordinates,

(c) $Y\left(K_{r}\right)$ denotes the fiber of $\pi_{r-1}$,

(d) $\rho_{i}$ is a projection defined below in terms of normal coordinates,

(e) $\mathscr{R}_{r}(i-1)$ denotes the fiber of $\rho_{i}$.

Proof. To begin consider a general point in $Y\left(k_{1}(1), \cdots, k_{m}(m)\right)$. It has the form

$$
\left[D_{1}, N_{1}\right]\left[D_{2}, N_{2}\right] \cdots\left[D_{m}, N_{m}\right]
$$

As we have seen, each $\left[D_{i}, N_{i}\right]$ can be uniquely written in the form $\left[D_{i}, M_{i}+R_{i}\right]$ where $D_{i}^{-1}(z) R_{i}(z) \rightarrow 0$ as $z \rightarrow \infty$. Thus, there is a differential surjection

$$
\pi_{1}: Y\left(k_{1}(1), \cdots, k_{m}(m)\right) \rightarrow \mathscr{R}_{1}
$$

given by projecting each $\left[D_{i}, N_{i}\right]$ to $\left[D_{i, i}(i), R_{i, i}(i)\right]$ where $R_{i, i}(i)$ is the bottom $\left(j_{i}-j_{i-1}\right) \times\left(j_{i+1}-j_{i}\right)$ block of $R_{i}$. The homotopy equivalence 
between $\mathscr{R}_{1}$ and $Y\left(L_{1}\right)$ follows from the normal form computations of $\S 4$. Furthermore, the map $\pi_{1}$ is, in fact, a submersion and, exactly as in $[11,5.1]$ it can be proved that $\pi_{1}$ is a fibering. (The argument proceeds by embedding $Y\left(k_{1}(1), \cdots, k_{m}(m)\right)$ into an appropriate product of symmetric products $S P^{\lambda_{i}}(\mathbb{C}) \subset S P^{\lambda_{i}}\left(\mathbb{C P}^{1}\right)$. The embedding includes $Y\left(k_{1}(1), \cdots, k_{m}(m)\right)$ as the complement of a closed set. By thickening this set and taking complements we see that $Y\left(k_{1}(1), \cdots, k_{m}(m)\right)$ deformation retracts onto a compact manifold pair $(Y, \partial Y)$ such that $\pi_{1} \mid \partial Y$ is also a smooth surjection. But from this the result follows.) It remains to identify the fiber and to show that $\mathscr{R}_{1}$ is an iterated fibration.

Before doing this let us consider some simple cases in order to anticipate the arguments for the general case. Therefore, we begin with the case $m=2$ by projecting the strata $Y(K)=Y\left(k_{1}(1), k_{1}(2), k_{2}(2)\right)$ onto the properly based factors of both bottom rows:

$$
\pi_{1}: Y\left(k_{1}(1), k_{1}(2), k_{2}(2)\right) \rightarrow \mathscr{R}_{1} \simeq Y\left(k_{1}(1), 0, k_{2}(2)\right) .
$$

Explicitly, in normal coordinates with the notation of $\S 4$,

$$
\pi_{1}\left([A, B],\left(\begin{array}{ll}
C & E \\
0 & F
\end{array}\right)\left(\begin{array}{c}
G \\
H
\end{array}\right)\right)=([A, R],[F, H]) .
$$

Using normal forms it is easy to verify that $Y\left(k_{1}(1), 0, k_{2}(2)\right)$ is the total space of a fibration

$$
\operatorname{Rat}_{k_{1}(1)}\left(\mathbb{G}_{j_{1}, j_{2}-j_{1}}\right) \rightarrow Y\left(k_{1}(1), 0, k_{2}(2)\right) \rightarrow \operatorname{Rat}_{k_{2}(2)}\left(\mathbb{G}_{j_{2}-j_{1}, n-j_{2}}\right) .
$$

This fibration is not topologically a product and the twisting is discussed in $\S \S 9$ and 10 .

At this point we identify the fiber of $\pi_{1}$, which we denote by $Y\left(k_{1}(2)\right)$. The fiber consists of $V=A_{0}^{-1} M, C, E$, and $G$ such that the left-hand side of (6.3) is an element of $Y(K)$. Recall that, as shown in [11], if there is no $[A, B]$ term, the projection

$$
\left(\begin{array}{ll}
C & E \\
0 & F
\end{array}\right)\left(\begin{array}{c}
G \\
H
\end{array}\right) \mapsto\left(\begin{array}{lll}
0 & F & H
\end{array}\right)
$$

is also a fibration. Recall also that, in this simpler case, with no $[A, B]$ term, the fiber can be identified with a entire copy of $\operatorname{Rat}_{k_{1}(2)}\left(\mathbb{G}_{j_{1}, n-j_{2}}\right)$. Since the presence of the $[A, B]$ term adds additional constraints, it is no longer true that the $[C, E, G]$ term itself can be identified with a Rat space. However, the freedom to vary the $V=A^{-1} M$ term precisely compensates for this difficulty. More precisely, the fiber of $\pi_{1}$ can be identified with a copy of $\operatorname{Rat}_{k_{1}(2)}\left(\mathbb{G}_{j_{1}, n-j_{2}}\right)$ via the homotopy equivalence

$$
\pi_{1}^{-1}\left(\left[A_{0}, R_{0}\right],\left[F_{0}, H_{0}\right]\right)=\left[C, E+V F_{0}, G+V H_{0}\right],
$$

so $Y\left(k_{1}(2)\right) \simeq \mathscr{R}_{2}$ in this case. 
Notice that the basing equations (4.4) and (4.5), Lemma 4.6 and Theorem 4.13 imply that the fiber $\{V, C, E, G\}$ is equivalent to the space of matrices $\left[C, E+V F_{0}, G+V H_{0}\right]$ which are based with respect to $C^{-1}$. Next, the fact that the right-hand side of (6.4) has full rank for all $z$ and hence represents an element of $\operatorname{Rat}_{k_{1}(2)}\left(\mathbb{G}_{j_{1}, n-j_{2}}\right)$ follows from the fact that $p_{2}(f)$ is a based map and from multiplying the bottom block row $\left[0, F_{0}, H_{0}\right]$ by $V$ and adding it to the top block row of $p_{2}(f)$ to obtain $\left[C, E+V F_{0}, G+V H_{0}\right]$.

\section{Remarks 6.5.}

(a) It is easier to use (4.5) rather than (4.2) here, although one could equivalently identify the fiber with elements of the form $\left[C, E+V F_{0}\right.$, $\left.P\left(G+E F_{0}^{-1} H_{0}\right)\right]$.

(b) The argument in the previous paragraph shows that the assignment of points in the fiber with [C,E+VF,$\left.G+V H_{0}\right]$ maps the fiber of $\pi_{1}$ into $\operatorname{Rat}_{k_{1}(2)}\left(\mathbb{G}_{j_{1}, n-j_{2}}\right)$ but does not show this assignment is surjective (even up to homotopy). However, by examining the fiber over an appropriately chosen point we can identify it with $\operatorname{Rat}_{k_{1}(2)}\left(\mathbb{G}_{j_{1}, n-j_{2}}\right)$ explicitly. Perhaps the best choices of matrices to fix this point are

$$
\begin{aligned}
& {\left[A_{0}, R_{0}\right]=\left(\begin{array}{cccc}
1 & \cdots & 0 & 0 \\
0 & \cdots & 0 & 0 \\
\vdots & \ddots & \vdots & \vdots \\
0 & \cdots & 1 & 0 \\
0 & \cdots & 0 & (z-1)^{k_{1}(2)}
\end{array}\right),\left(\begin{array}{ccc}
0 & \cdots & 0 \\
\vdots & \ddots & \vdots \\
0 & \cdots & 0 \\
\vdots & \ddots & \vdots \\
1 & \cdots & 0
\end{array}\right),} \\
& {\left[F_{0}, H_{0}\right]=\left(\begin{array}{cccc}
1 & \cdots & 0 & 0 \\
0 & \cdots & 0 & 0 \\
\vdots & \ddots & \vdots & \vdots \\
0 & \cdots & 1 & 0 \\
0 & \cdots & 0 & z^{k_{2}(2)}
\end{array}\right),\left(\begin{array}{ccc}
0 & \cdots & 0 \\
\vdots & \ddots & \vdots \\
0 & \cdots & 0 \\
\vdots & \ddots & \vdots \\
1 & \cdots & 0
\end{array}\right)}
\end{aligned}
$$

It then follows that

1. Only the last column of $E$ may be nonzero.

2. The basing condition implies that

$$
C^{-1}\left(\begin{array}{c}
e_{1, j_{2}-j_{1}}+v_{1, j_{2}-j_{1}} z^{k_{2}(2)} \\
e_{2, j_{2}-j_{1}}+v_{2, j_{2}-j_{1}} z^{k_{2}(2)} \\
\vdots \\
e_{j_{1}, j_{2}-j_{1}}+v_{j_{1}, j_{2}-j_{1}} z^{k_{2}(2)}
\end{array}\right)
$$

tends to zero as $z \rightarrow \infty$. 
3. The last $n-j_{2}-1$ columns of $P\left(G-E F_{0}^{-1} H_{0}\right)$ are precisely the last $n-j_{2}-1$ columns of $G$.

4. The basing condition determines the first column of $P\left(G-E F_{0}^{-1} H_{0}\right)$ via

$$
C^{-1}\left(\begin{array}{c}
g_{1,1}+e_{1, j_{2}-j_{1}} z^{-k_{2}(2)} \\
g_{2,1}+e_{2, j_{2}-j_{1}} z^{-k_{2}(2)} \\
\vdots \\
g_{j_{1}, 1}+e_{j_{1}, j_{2}-j_{1}} z^{-k_{2}(2)}
\end{array}\right)
$$

tends to zero as $z \rightarrow \infty$.

One can then generalize the arguments of [11, 5.6-5.11] to construct an explicit homotopy equivalence of $\pi_{1}^{-1}(*)$ with $\mathrm{Rat}_{k_{1}(2)}\left(\mathbb{G}_{j_{1}, n-j_{2}}\right)$ in this case. Notice that the first $j_{2}-j_{1}-1$ columns of $V$ satisfy only $C^{-1} V^{s} \rightarrow 0$ as $z \rightarrow \infty$ but do not affect the rank condition as they merely add vectors in the span of $\left[0, F_{0}, H_{0}\right]$. Thus, these columns of the $V$ matrix do not affect the homotopy type of the fiber of $\pi_{1}$; rather their role is to control the dimension of the strata in a manner compatible with the normal bundle discussion of the previous section.

We now consider the case $m=3$ in order to exhibit an additional subtlety that must be taken into account. Recall we can write an arbitrary element $f$ of $Y(K)=Y\left(k_{1}(1), k_{1}(2), k_{2}(2), k_{1}(3), k_{2}(3), k_{3}(3)\right)$ as

$$
\left[D_{1,1}(1), R_{1,2}(1)+M_{1,2}(1)\right]\left[D_{2}, N_{2}\right]\left[D_{3}, N_{3}\right]
$$

where

$$
\left[D_{2}, N_{2}\right]=\left[\begin{array}{ccc}
D_{1,1}(2) & E_{1,2}(2) & R_{1,3}(2)+M_{1,2}(2) \\
0 & D_{2,2}(2) & R_{2,3}(2)+M_{2,3}(2)
\end{array}\right]
$$

and

$$
\left[D_{3}, N_{3}\right]=\left[\begin{array}{cccc}
D_{1,1}(3) & E_{1,2}(3) & E_{1,3}(3) & R_{1,4}(3) \\
0 & D_{2,2}(3) & E_{2,3}(3) & R_{2,4}(3) \\
0 & 0 & D_{3,3}(3) & R_{3,4}(3)
\end{array}\right]
$$

Here the $R$ and $M$ matrices are as in $\S 4$. We project

$$
\pi_{1}: Y(K) \rightarrow \mathscr{R}_{1} \simeq Y\left(k_{1}(1), 0, k_{2}(2), 0,0, k_{3}(3)\right)
$$

via the assignment

$$
\pi_{1}(f)=\left(\left[D_{1,1}(1), R_{1,2}(1)\right],\left[D_{2,2}(2), R_{2,3}(2)\right],\left[D_{3,3}(3), R_{3,4}(3)\right]\right) \text {. }
$$

Again, it is routine to see that $Y\left(k_{1}(1), 0, k_{2}(2), 0,0, k_{3}(3)\right)$ is the total space of a fibration with fiber $\operatorname{Rat}_{k_{1}(1)}\left(\mathbb{G}_{j_{1}, j_{2}-j_{1}}\right)$ and base 
$Y\left(0,0, k_{2}(2), 0,0, k_{3}(3)\right)$. But we have already seen this base space is itself the total space of a fibration with fiber $\operatorname{Rat}_{k_{2}(2)}\left(\mathbb{G}_{j_{2}-j_{1}, j_{3}-j_{2}}\right)$ and base $\operatorname{Rat}_{k_{3}(3)}\left(\mathbb{G}_{j_{3}-j_{2}, n-j_{3}}\right)$.

As $\pi_{1}$ is a fibration, with fiber $Y\left(K_{2}\right)$, we project again

$$
\pi_{2}: Y\left(K_{2}\right) \rightarrow \mathscr{R}_{2} \simeq Y\left(0, k_{1}(2), 0,0, k_{2}(3), 0\right),
$$

where the base is the total space of a fibration with fiber $\operatorname{Rat}_{k_{1}(2)}\left(\mathbb{G}_{j_{1}, j_{3}-j_{2}}\right)$ and base $\operatorname{Rat}_{k_{2}(3)}\left(\mathbb{G}_{j_{2}-j_{1}, n-j_{3}}\right)$ via the assignment which projects onto the following two elements:

$$
\left[D_{1,1}(2), \bar{R}_{1,2}(2), \bar{R}_{1,3}(2)\right],
$$

$$
\left.\left[D_{2,2}(3), E_{2,3}(3)+V_{2,3}(2)\left(D_{3,3}(3)\right)_{0}, R_{2,3}(2)-V_{2,3}(3) R_{3,4}(3)\right)_{0}\right] \text {. }
$$

\section{Remarks 6.13.}

(a) $V_{i, j}(k)=D_{i, i}^{-1}(k) M_{i, j}(k)$ as in $\S 4$.

(b) We have written $E_{1,2}+V_{1,2}(1) D_{2,2}(2)$ as $\bar{R}_{1,2}(2)+\bar{M}_{1,2}$ (2) where $D_{1,1}^{-1}(2) \bar{R}_{1,2}(2) \rightarrow 0$ as $z \rightarrow \infty$. We shall need $\bar{V}_{1,2}(2)=D_{1,1}^{-1}(2) \bar{M}_{1,2}(2)$ to identify the iterated fiber of $\pi_{2}$.

(c) We have written $R_{1,3}(2)+V_{1,2}(1) R_{2,3}(2)$ as $\bar{R}_{1,3}(2)+\bar{M}_{1,3}(2)$ where $D_{1,1}^{-1}(2) \bar{R}_{1,3}(2) \rightarrow 0$ as $z \rightarrow \infty$. Again, we shall need $\bar{V}_{1,3}(2)=$ $D_{1,1}^{-1}(2) \bar{M}_{1,3}$ (2) to identify the iterated fiber of $\pi_{2}$.

(d) Notice that, in general, the matrix

$$
\left[D_{1,1}(2), E_{1,2}(2)+V_{1,2} D_{2,2}^{-1}(2), R_{1,3}(2)+V_{1,2} D_{2,2}^{-1}(2)\right]
$$

is not properly based. However, just as in the proof of Theorem 4.19, we use the based piece of (6.14) to project onto, while the remaining degrees of freedom are saved to base a projection at the next stage of the induction. It is precisely this iterated projection onto the based piece which makes it possible to identify all the fibres in the general case.

By using the basing conditions, Theorems 4.13 and 4.19 , just as in the case $m=2$, we see that $\pi_{2}$ is a smooth surjection and fibration. Again, the fact that each $p_{i}(f)$ is a based map and that (6.11) and (6.12) are obtained by adding multiples of the bottom block row to the next ensure that, for all $z,(6.11)$ and (6.12) have full rank. Next, the basing conditions imply that $\pi_{2}$ maps $Y\left(K_{2}\right)$ into $Y\left(0, k_{1}(2), 0,0, k_{2}(3), 0\right)$. The fact that $\pi_{2}$ is onto follows again by considering the inverse image of the point given by defining the relevant $D_{a, a}(b)_{0}, E_{a, b}(c)_{0}$, and $R_{a, b}(c)_{0}$ matrices as in (6.6) and (6.7). 
Finally, $Y\left(K_{3}\right)$, the fibre of $\pi_{2}$, is homotopy equivalent to Rat $_{k_{1}(3)}\left(\mathbb{G}_{j_{1}, n-j_{3}}\right)$. Again, this can be checked via the assignment

$$
\pi_{3}(f)=\left(\left[D_{1,1}(3), E_{1,2}(3)+\bar{V}_{1,2}(2) D_{2,2}(3)_{0}, Y, Z\right]\right)
$$

for $f \in Y\left(K_{3}\right)$ where

$$
\begin{aligned}
Y= & E_{1,3}(3)+\bar{V}_{1,2}(2) E_{2,3}(3)_{0}+\bar{V}_{1,3}(2) D_{3,3}(3)_{0} \\
& +V_{1,2}(1)\left[D_{2,2}(3)_{0} E_{2,3}(3)_{0}+V_{2,3}(2)\left(D_{3,3}(3)\right)_{0}\right]
\end{aligned}
$$

and

$$
\begin{aligned}
Z= & R_{1,4}(3)+P\left(D_{1,1}^{-1}(2)_{0} D_{2,2}^{-1}(2)_{0}\left[R_{2,4}(3)_{0}+V_{2,3}(2) R_{3,4}(3)_{0}\right]\right) \\
& +V_{1,2}(1)\left[D_{2,2}(2)_{0} R_{2,4}(3)_{0}+V_{2,3}(2) R_{3,4}(3)_{0}\right]
\end{aligned}
$$

Once again, since the projection was constructed using iterates of the basing conditions, it follows immediately that the fiber is a subset of the appropriate Rat space. Next, we may use the full freedom of the various $V$ matrices to fill out the copy of $\operatorname{Rat}_{k_{1}(3)}\left(\mathbb{G}_{j_{1}, n-j_{3}}\right)$. This computation is basically identical to the computations in the proof of Theorem 4.19.

The extension to the general case and the complete proof of Proposition 6.1 are now clear. The proof is an induction on $m$ with the case $m=1$ 5.1 of [11]) while the cases $m=2$ and $m=3$ are given above. The fact that each $\pi_{r}$ and $\rho_{s}$ are fibrations follows exactly as in the Grassmannian case as has been indicated.

It remains to identify the homotopy type of the fiber which can be done by analyzing the projection above the point defined by iterates of $(6.6)$ and (6.7). This is carried out exactly as above by the "peeling" projections onto the modified block rows of the $\left[D_{t}, N_{t}\right]$ matrices where, at each stage, the extra degrees of freedom, as captured in the $V_{a, b}(c)$ matrix blocks, are used to bring the block rows to based form. More precisely, the fundamental basing equations $\lim _{z \rightarrow \infty}\left(P_{s}^{-1} \mathscr{J}_{s+t}(s)\right)=0$ are all "divisible" by the appropriate $D_{a, a}(r)$ matrices. By that we mean that these fundamental basing equations, which appear as rational equations involving certain $D_{a, a}(r)$ matrices, can be written as polynomial equations in the $V$ 's. But these polynomial equations can be used to explicitly write down the projection maps $\pi_{r}$. q.e.d.

Just as in Theorem 2.8 the iterate fibration in Proposition 6.1 yields

Proposition 6.15. There is a filtration of each stratum $Y(K)$ so that the $E^{2}$ term in the resulting spectral sequence converging to $H_{*}(Y(K) ; \mathbb{F})$ 
for $\mathbb{F}$ any field is a tensor product of suspensions of terms of the form $H_{*}\left(\operatorname{Rat}_{k_{i-r+1}(i)}\left(\mathbb{G}_{j_{i-r+1}-j_{i-r}, j_{i+1}-j_{i}}\right) ; \mathbb{F}\right)$ 's.

Furthermore, we may combine Proposition 2.6, Theorem 2.8 and Proposition 6.1 to obtain

Proposition 6.16. There is a filtration of each stratum $Y(K)$ so that the $E^{2}$ term in the resulting spectral sequence converging to $H_{*}(Y(K) ; \mathbb{F})$ for $\mathbb{F}$ any field is a tensor product of suspensions of terms of the form $H_{*}\left(\operatorname{Rat}_{k(a, b, c)}\left(\mathbb{C P}\left(j_{r}-j_{r-1}\right)\right) ; \mathbb{F}\right)$ 's.

As done in [11] for $\operatorname{Rat}_{k}\left(\mathbb{G}_{n, m}\right)$ and in [2] for moduli spaces of instantons it is possible to use the filtration of $\operatorname{Rat}_{C}(\mathbb{F}(J))$ by the $Y(K)$ to construct a Leray spectral sequence converging to a filtration of $H_{*}\left(\operatorname{Rat}_{C}(\mathbb{F}(J))\right)$ with $E^{1}$ term computable in terms of the (suspended) homology of the $Y(K)$ 's. However, differentials in this Leray spectral sequence are hard to track and the filtration used is not compatible with the usual Serre filtration on $\Omega^{2} \mathbb{F}(J)$. Consequently, in order to do actual calculations it is necessary to introduce a further filtration on our spaces. We address this problem in the next section.

\section{A new filtration and the stability theorem}

We now modify our stratification of $\operatorname{Rat}_{C}(\mathbb{F}(J))$ to make the previous geometric analysis more compatible with the forgetful map $l(J ; C)$ : $\operatorname{Rat}_{C}(\mathbb{F}(J)) \rightarrow \Omega^{2} \mathbb{F}(J)$.

Definition 7.1. For each $1 \leq k \leq c_{1}$ let

$$
X(k)=\bigcup_{K=\left(k, k_{1}(2), \cdots, k_{m}(m)\right)} Y(K) .
$$

Thus, $X(k)$ is the union of all strata $Y(K) \subset$ Rat $_{C}(\mathbb{F}(J))$ such that the $k_{1}(1)$ entry of $K$ is $k$. Notice here that for some values of the total Chern class $X(K)$ may be empty. For example, if $m=2, c_{1}=100$, and $c_{2}=5$, then $X(k)$ is nonempty only for $k$ between 95 and 100 . Of course, there is a $k_{0}$ depending on $C$ and $J$ such that

$$
\operatorname{Rat}_{C}(\mathbb{F}(J))=\coprod_{k=k_{0}}^{c_{1}} X(k),
$$

where the right-hand side is the disjoint union of nonempty strata. Furthermore, for each $k_{0} \leq k \leq c_{1}$, the stratum $Y(K)$ of $\operatorname{Rat}_{C}(\mathbb{F}(J))$ with 
$k_{1}(1)=k, k_{1}(2)=c_{1}-k, k_{2}(2)=c_{2}-c_{1}+k, k_{r}(r)=c_{r}$ for $3 \leq r \leq m$, and $k_{s}(r)=0$ otherwise, is a generic open set in $X(k)$ (open in $X(k)$ and not, unless $k=c_{1}$, open in $\left.\operatorname{Rat}_{C}(\mathbb{F}(J))\right)$. The lexicographic order on the set of all multipartitions $K$ gives that set a poset structure, and, as the choice of index associated to the generic open set is an extremal element, it follows that each $X(k)$ is a complex submanifold of $\operatorname{Rat}_{C}(\mathbb{F}(J))$. But then it also follows that the proof of Proposition 5.5 extends directly to show that the normal bundles of the $X(k)$ 's in $\operatorname{Rat}_{C}(\mathbb{F}(J))$ are all complex. Summarizing we have

Proposition 7.2. There is a stratification of $\operatorname{Rat}_{C}(\mathbb{F}(J))$ obtained from the stratification of Definition 5.2 by taking unions as in Definition 7.1. This coarser stratification consists of strata $X(k)$ which are complex submanifolds of $\operatorname{Rat}_{C}(\mathbb{F}(J))$ such that the normal bundles $\nu(X(k))$ are complex bundles. Here $k_{0}=\max \left(0, c_{1}-c_{2}\right) \leq k \leq c_{1}$.

We now justify the introduction of this new stratification. Consider the following commutative diagram:

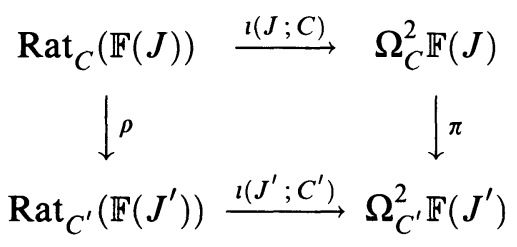

where

(a) $J^{\prime}$ is obtained from $J$ by forgetting the $j_{1}$ entry,

(b) $C^{\prime}$ is obtained from $C$ by forgetting the $c_{1}$ entry,

(c) both $\rho$ and $\pi$ are the forgetful maps induced by forgetting the smallest flag,

(d) recall both $l(J ; C)$ and $l\left(J^{\prime} ; C^{\prime}\right)$ are the natural inclusions induced by forgetting the holomorphic structure.

While $\rho$ is not necessarily onto (this depends on the actual values of $C), \rho$ is always an open map. Of course, while $\pi$ is a fibration of two-fold loop spaces with fibre $\Omega^{2} \mathbb{G}_{j_{1}, j_{2}-j_{1}}, \rho$ is not a fibration over its image. The point is that the inverse image of $\rho$ varies as one moves around in the base space. However, we can thicken the individual strata $X(k) \subset \operatorname{Rat}_{C}(\mathbb{F}(J))$ to open regular neighborhoods $X_{t}(k)$ that have the same homotopy type as $X(k)$ by using the complex normal bundles $\nu(X(k))$. But the arguments in $\S 6$ show that, when restricted to each $X_{t}(k), \rho$ is a fibration, with fiber homotopy equivalent to $\operatorname{Rat}_{k}\left(\mathbb{G}_{j_{1}, j_{2}-j_{1}}\right)$. 
Thus, for each $k_{0} \leq k \leq c_{1}$, we have the following commutative diagram where the vertical maps are both fibrations:

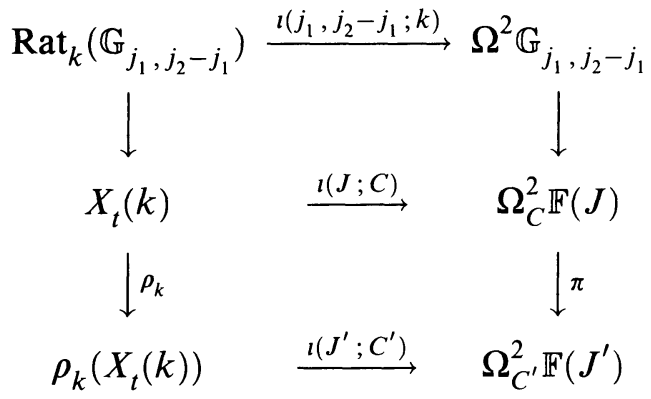

Here $\rho_{k}$ is the restriction of $\rho$ to $X_{t}(k)$.

Since the thickened open strata $X_{t}(k)$ cover $\operatorname{Rat}_{C}(\mathbb{F}(J))$ and $\rho$ is an open map, the image strata $\rho_{k}\left(X_{t}(k)\right)$ cover the open image of $\rho$ in $\operatorname{Rat}_{C^{\prime}}\left(\mathbb{F}\left(J^{\prime}\right)\right)$. Therefore, in homology, we may consider cells that are subordinate to these open covers. In this way we see that, while the inverse image of $\rho$ varies over different points, $\rho$ restricted to each individual cell is always a fibration. We now filter $\operatorname{Rat}_{C}(\mathbb{F}(J))$ using the inverse images, under $\rho$, of cells in $\operatorname{Rat}_{C^{\prime}}\left(\mathbb{F}\left(J^{\prime}\right)\right)$ to obtain a Leray spectral sequence converging to a filtration of $H_{*}\left(\operatorname{Rat}_{C}(\mathbb{F}(J)) ; A\right)$. The point is that, by construction, the new filtration arising from the inverse images of cells in (7.4) is manifestly compatible with the usual Serre filtration on the fibration $\pi$. Thus, we have

Theorem 7.5. The Leray spectral sequence associated to the filtration above converging to $H_{*}\left(\operatorname{Rat}_{C}(\mathbb{F}(J)) ; A\right)$ has $E^{2}$ term isomorphic to

$$
E_{s, t}^{2} \cong H_{s}\left(\operatorname{Rat}_{C^{\prime}}\left(\mathbb{F}\left(J^{\prime}\right)\right) ; H_{t}\left(\operatorname{Rat}_{k(\alpha)}\left(\mathbb{G}_{j_{1}, j_{2}-j_{1}}\right) ; A\right)\right) \text {. }
$$

Here

(a) $C^{\prime}=\left(c_{2}, \cdots, c_{m}\right)$,

(b) $J^{\prime}=\left(j_{2}, \cdots, j_{m}\right)$,

(c) $0 \leq k(\alpha) \leq c_{1}$. The value of $k(\alpha)$ over any homology class in the base $\alpha$ is determined by the stratum $X(k)$ that carries $\alpha$.

Furthermore, the natural inclusion $\imath(J ; C)$ induces a map of spectral sequences

$$
l(J ; C)_{r}: E^{r}\left(\operatorname{Rat}_{C}(\mathbb{F}(J)) ; \text { Leray }\right) \rightarrow E^{r}\left(\Omega^{2} \mathbb{F}(J) ; \text { Serre }\right) .
$$

In particular, differentials are natural in these two spectral sequences.

Proof. The preceding discussion shows that the Leray spectral sequence associated to this new filtration of $\operatorname{Rat}_{C}(\mathbb{F}(J))$ is naturally filtration pre- 
serving with respect to $l(J ; C)$ and the Serre fibration on $\pi$. Thus, it remains to identify the $E^{2}$ term. As we only need to consider cells subordinate to the open cover of the $X_{t}(k)$, this follows immediately from the fibrations given in (7.4) for $k_{0} \leq k \leq c_{1}$.

Remark 7.8. As mentioned at the end of $\S 6$ the stratification of $\operatorname{Rat}_{C}(\mathbb{F}(J))$ by the $Y(K)$ can be used just as in [11] and [2] to construct a filtration of $\operatorname{Rat}_{C}(\mathbb{F}(J))$ with associated Leray spectral sequence $E^{r}(Y(K))$ converging to a filtration of $H_{*}\left(\operatorname{Rat}_{C}(\mathbb{F}(J))\right)$. However, the Leray spectral sequence $E^{r}(Y(K))$ constructed in this way is very different from the Leray spectral sequence constructed in Theorem 7.5.

As an immediate corollary of Theorem 7.5 we have the finite stage stability theorem for arbitrary flag manifolds stated in the introduction.

Corollary 7.9 (Flag Stability Theorem). Given $\left(j_{1}, \cdots, j_{m}\right), k>0$, there is an m-tuple of positive integers $\left(s_{1}(k), \cdots, s_{m}(k)\right)$ so that for any $C$ with $c_{i} \geq s_{i}(k)$ then the inclusion into the second loop space, $\operatorname{Rat}_{C}(\mathbb{F}(J)) \rightarrow \Omega_{C}^{2}(\mathbb{F}(J))$, is a homology equivalence through dimension at least $k$. Furthermore, if $J$ is not $(1,2, \cdots, m)$, representing the complete flag of length $n=m+1$, then both $\operatorname{Rat}_{C}(\mathbb{F}(J))$ and $\Omega^{2} \mathbb{F}(J)$ are simply connected so that $l(C, J)$ is a homotopy equivalence through the same range.

Proof. The homology results follows immediately from induction using Theorem 7.5 and the stability theorems for $\operatorname{Rat}_{*}(\mathbb{C P}(n))$ and $\operatorname{Rat}_{*}\left(\mathbb{G}_{i, j}\right)$ proved in [14] and [10] and sharpened in [5] and [11]. The statement on fundamental groups is well known.

Remark 7.10. While the stability theorem for general flags is identical in spirit to the special case for Grassmannians, it is a different story above the range of stability. In fact, the situation for general flag manifolds is considerably more subtle than for Grassmannians; for example, the map in homology $l(k ; i, j): H_{*}\left(\operatorname{Rat}_{k}\left(\mathbb{G}_{i, j}\right) ; A\right) \rightarrow H_{*}\left(\Omega_{k}^{2} \mathbb{G}_{i, j} ; A\right)$ was shown to be injective for every $k \geq 1$ in [11]. However, we will show in $\S 11$ that this is not true already in the case of general 2-stage flag manifolds $\mathrm{Rat}_{1,1}\left(\mathbb{F}\left(j_{1}, j_{2} ; n\right)\right)$.

\section{The space $\operatorname{Rat}_{1}\left(\mathbb{G}_{n, m}\right)$}

In this section we analyze the geometry of $\operatorname{Rat}_{1}\left(\mathbb{G}_{n, m}\right)$ by identifying $\operatorname{Rat}_{1}\left(\mathbb{G}_{n, m}\right)$, up to homeomorphism, with a well-known space and, as a corollary, determine its homology. Then in the next two sections we extend this analysis to the $\operatorname{Rat}_{1, \ldots, 1}(\mathbb{F}(J))$. 
We consider the space of $n \times n$ matrices $\mathscr{M}_{A}$ with coefficients in $\mathbb{C}[z]$ and determinant $z-A$ under row equivalence. More exactly, for $\alpha \in \mathscr{M}_{A}$, we set

$$
\alpha \sim U \alpha,
$$

where $U \in \mathrm{GL}_{n}(\mathbb{C}[z])$. This action $\mathrm{GL}_{n}(\mathbb{C}[z]) \times \mathscr{M}_{A} \rightarrow \mathscr{M}_{A}$ is continuous, and we write $\mathrm{GL}_{n}(\mathbb{C}[z]) \backslash \mathscr{M}_{A}$ for the orbit space.

Remark 8.1. Any such $\alpha$ is uniquely equivalent to an upper triangular matrix

$$
B_{i}\left(a_{1, i}, \cdots, a_{i-1, i}\right)=\left(\begin{array}{cccccccc}
1 & 0 & \cdots & a_{1, i} & 0 & \cdots & 0 \\
0 & 1 & \cdots & a_{2, i} & 0 & \cdots & 0 \\
\cdot & \cdot & \cdots & \cdot & \cdot & \cdots & \cdot \\
0 & 0 & \cdots & a_{i-1, i} & 0 & \cdots & 0 \\
0 & 0 & \cdots & z-A & 0 & \cdots & 0 \\
0 & 0 & \cdots & 0 & 1 & \cdots & 0 \\
\cdot & \cdot & \cdots & \cdot & \cdot & \cdots & \cdot \\
0 & 0 & \cdots & 0 & 0 & \cdots & 1
\end{array}\right),
$$

where $a_{1, i}, \cdots, a_{i-1, i} \in \mathbb{C}$. In particular, note that even though both $\mathscr{M}_{A}$ and $\mathrm{GL}_{n}(\mathbb{C}[z])$ are infinite dimensional, the orbit space is finite dimensional, in fact an $(n-1)$-dimensional complex manifold. Precisely, we have

Theorem 8.2. $\quad \mathrm{GL}_{n}(\mathbb{C}[z]) \backslash \mathscr{M}_{A} \simeq \mathbb{C P}^{n-1}$.

Proof. Consider the matrices

$$
\begin{aligned}
& \quad\left(\begin{array}{ccccc}
z-A & 0 & \cdots & 0 & 0 \\
a_{2,1} & -1 & \cdots & 0 & 0 \\
\vdots & \vdots & \ddots & \vdots & \vdots \\
a_{n-1,1} & 0 & \cdots & -1 & 0 \\
a_{n, 1} & 0 & \cdots & 0 & -1
\end{array}\right)=A_{1}\left(a_{2,1}, \cdots, a_{n, 1}\right), \\
& \left(\begin{array}{cccccc}
-1 & 0 & \cdots & a_{1, i} & \cdots & 0 \\
0 & -1 & \cdots & a_{2, i} & \cdots & 0 \\
\vdots & \vdots & \ddots & \vdots & \ddots & \vdots \\
0 & 0 & \cdots & z-A & \cdots & 0 \\
\vdots & \vdots & \ddots & \vdots & \ddots & \vdots \\
0 & 0 & \cdots & a_{n, i} & \cdots & -1
\end{array}\right)=A_{i}\left(a_{1, i}, \ldots, a_{i-1, i}, a_{i+1, i}, \ldots, a_{n, i}\right) .
\end{aligned}
$$

Note that if, for example, $a_{i, 1} \neq 0$, then we have directly

$$
A_{1}\left(a_{2,1}, \cdots, a_{n, 1}\right) \sim A_{i}\left(\frac{1}{a_{i, 1}}, \frac{a_{2,1}}{a_{i, 1}}, \cdots, \frac{a_{i-1,1}}{a_{i, 1}}, \frac{a_{i+1,1}}{a_{i, 1}}, \cdots, \frac{a_{n, 1}}{a_{i, 1}}\right) .
$$


In particular, if $a_{n, 1} \neq 0$ the resulting matrix $A_{n}(\cdots)$ is in normal form except for signs. Likewise, if $a_{i, 1} \neq 0$ but $a_{j, 1}=0$ for $j>i$, then $A_{1}(\cdots) \sim A_{i}(\cdots)$, and $A_{i}(\cdots)$, except for signs is in normal form, $\left(A_{i}\left(a_{1}, \cdots, a_{i-1}\right) \sim B_{i}\left(-a_{1},-a_{2}, \cdots,-a_{i-1}\right)\right)$. On the other hand the relation (8.3) above is exactly the equivalence relation that holds among the charts of $\mathbb{C P}^{n-1}$, and from these two remarks the theorem follows easily. q.e.d.

Next we consider the situation for $\operatorname{Rat}_{1}\left(\mathbb{G}_{n, m}\right)$, the space of based holomorphic maps $f: S^{2} \rightarrow \mathbb{G}_{n, m}$ satisfying $\left\langle f^{*}\left(c_{1}\right),\left[S^{2}\right]\right\rangle=1$ where $c_{1}$ is the first Chern class. Here, any element is uniquely written as a pair $[D, N]$ where $D$ is an $n \times n$-upper triangular matrix of the form

$$
D=\left(\begin{array}{ccccccc}
1 & \cdots & 0 & a_{1, i} & 0 & \cdots & 0 \\
0 & \cdots & 0 & a_{2, i} & 0 & \cdots & 0 \\
\vdots & \ddots & \vdots & \vdots & \vdots & \ddots & \vdots \\
0 & \cdots & 1 & a_{i-1, i} & 0 & \cdots & 0 \\
0 & \cdots & 0 & z-A & 0 & \cdots & 0 \\
0 & \cdots & 0 & 0 & 1 & \cdots & 0 \\
\vdots & \ddots & \vdots & \vdots & \vdots & \ddots & \vdots \\
0 & \cdots & 0 & 0 & 0 & \cdots & 1
\end{array}\right),
$$

and, for such a $D, N$ is $n \times m$ and has the form

$$
N=\left(\begin{array}{ccc}
0 & \cdots & 0 \\
\vdots & \ddots & \vdots \\
N_{i, 1} & \cdots & N_{i, m} \\
0 & \cdots & 0 \\
\vdots & \ddots & \vdots \\
0 & \cdots & 0
\end{array}\right)
$$

with $N_{i, j} \in \mathbb{C}, \sum_{1}^{m}\left|N_{i, j}\right|^{2} \neq 0$.

Over $\mathbb{C P}^{n}$, we define the complex line bundle $\left(\xi^{j}\right)_{n}$, as the bundle given in homogeneous coordinates as follows:

$$
\left(a_{0}, \cdots, a_{n}, w\right) \sim\left(z a_{0}, \cdots, z a_{n}, z^{j} w\right), \quad z \in \mathbb{C}^{*}, \sum_{0}^{n}\left|a_{i}\right|^{2} \neq 0 .
$$

Corollary 8.6. $\operatorname{Rat}_{1}\left(\mathbb{G}_{n, m}\right)$ is homeomorphic to the product

$$
\mathbb{C} \times\left\{m\left(\xi^{-1}\right)_{n-1}\right\}^{*},
$$

where $m \xi$ denotes the $m$-fold Whitney bundle sum, and $\{m \xi\}^{*}$ denotes the complement of the zero section. 
Proof. This is a direct extension of the previous result. Precisely, write

$$
\begin{gathered}
\left.A_{1}\left(a_{2,1}, \cdots, a_{n, 1}\right),\left(\begin{array}{ccc}
N_{1,1} & \cdots & N_{1, m} \\
0 & \cdots & 0 \\
\vdots & \ddots & \vdots \\
0 & \cdots & 0
\end{array}\right)\right] \\
=A_{1}\left(a_{2,1}, \cdots, a_{n, 1}: N_{1,1}, \cdots, N_{1, m}\right)
\end{gathered}
$$

with a similar meaning for $\left[A_{i}\left(a_{1, i}, \cdots, a_{i-1, i}, a_{i+1,1}, \cdots, a_{n, i}: N_{i, 1}\right.\right.$, $\left.\left.\cdots, N_{i, m}\right)\right]$. Then it is easily seen that if $a_{i} \neq 0$ we have

$$
\begin{aligned}
& A_{1}\left(a_{2}, \cdots, a_{n}: N_{1,1}, \cdots, N_{1, m}\right) \\
& \quad \sim A_{i}\left(\frac{1}{a_{i}}, \frac{a_{2}}{a_{i}}, \cdots, \frac{a_{i-1}}{a_{i}}, \frac{a_{i+1}}{a_{i}}, \cdots, \frac{a_{n}}{a_{i}}: a_{i} N_{1,1}, \cdots, a_{i} N_{1, m}\right)
\end{aligned}
$$

with similar results in the other cases. From this the corollary follows.

Remark 8.7. Delchamps [6] first computed $H_{*}\left(\operatorname{Rat}_{1}\left(\mathbb{G}_{n, m}\right), \mathbb{Z}\right)$ using Morse theory. That computation was possible because, as Corollary 8.6 shows, $\operatorname{Rat}_{1}\left(\mathbb{G}_{n, m}\right)$ admits a $\mathrm{CW}$ decomposition where cells never occur in consecutive dimensions and hence there are no torsion classes in $H_{*}\left(\operatorname{Rat}_{1}\left(\mathbb{G}_{n, m}\right), \mathbb{Z}\right)$. However, for $k>1, H_{*}\left(\operatorname{Rat}_{k}\left(\mathbb{G}_{n, m}\right), \mathbb{Z}\right)$ is almost exclusively torsion [11] so the case $k=1$ is somewhat of an anomaly.

As a consequence of Corollary 8.6, Rat $_{1}\left(\mathbb{G}_{n, m}\right)$ is homotopy equivalent to the total space of the sphere bundle $S\left(m\left(\xi^{-1}\right)_{n-1}\right)$,

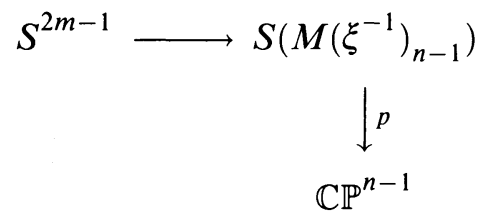

and, to compute its cohomology we can use the Gysin sequence, remembering that the Euler class of $m \xi^{-1}$ is $(-b)^{m}$, where $b$ is the usual generator for $H^{2}\left(\mathbb{C P}^{n-1} ; \mathbb{Z}\right)$. Putting this together, the Gysin sequence in our situation takes the form

$$
\begin{aligned}
\cdots \rightarrow H^{*}\left(\left\{m \xi^{-1}\right\}^{*} ; \mathbb{Z}\right) \stackrel{\delta}{\rightarrow} H^{*-2 m+1}\left(\mathbb{C P}^{n-1} ; \mathbb{Z}\right) \stackrel{\cup(-b)^{m}}{\longrightarrow} H^{*+1}\left(\mathbb{C P}^{n-1} ; \mathbb{Z}\right) \\
\stackrel{p^{*}}{\longrightarrow} H^{*+1}\left(\left\{m \xi^{-1}\right\}^{*} ; \mathbb{Z}\right) \longrightarrow \cdots
\end{aligned}
$$

As a consequence, for $n \leq m$ we have

Lemma 8.9. For $n \leq m$ there is a class $M \in H^{2 m+1}\left(\left\{m \xi^{-1}\right\}_{n-1} ; \mathbb{Z}\right)=$ $\mathbb{Z}$ and, as a ring we have that $H^{*}\left(\left\{m \xi^{-1}\right\}_{n-1} ; \mathbb{Z}\right)=\mathbb{Z}[b] / b^{n} \otimes E[M]$ where $b \in H^{2}\left(\left\{m \xi^{-1}\right\}_{n-1} ; \mathbb{Z}\right)$. 
We can obtain more detailed information about $S\left(m\left(\xi^{-1}\right)_{n-1}\right)$. Note that

$$
S\left(m\left(\xi^{-1}\right)_{n-1}\right)=S^{2 m-1} \times S^{2 n-1} / \mathscr{R}
$$

where $\mathscr{R}$ is the relation

$$
(\vec{\varphi}, \vec{\omega}) \sim\left(\zeta \vec{\varphi}, \zeta^{-1} \vec{\omega}\right), \zeta=e^{i \theta},
$$

and from the fact that this description is symmetric in $n$ and $m$, we have

Lemma 8.11. There is a homeomorphism $S\left(m\left(\xi^{-1}\right)_{n-1} \cong S\left(n\left(\xi^{-1}\right)_{m-1}\right)\right.$.

As $\mathbb{G}_{n, m}$ is antiholomorphically equivalent to $\mathbb{G}_{m, n}$ Lemma 8.11 should be no surprise. Furthermore, it is instructive to note that $(8.8)$ shows there can be no differentials in the spectral sequence of Theorem 2.8 for $H_{*}\left(\operatorname{Rat}_{1}\left(\mathbb{G}_{n, m}\right)\right)$ when $n \leq m$, and that when $n>m,(8.8)$ forces the obvious differentials in Theorem 2.8. These differentials for $n>m$ are also well known to occur in the spectral sequence for $H_{*}\left(\Omega^{2} \mathbb{G}_{n, m}\right)$.

\section{The geometry of $\operatorname{Rat}_{(1, \cdots, 1)}(\mathbb{F}(J))$}

In these next two sections we extend the analysis just given for $\operatorname{Rat}_{1}\left(\mathbb{G}_{n, m}\right)$ to flag manifolds. The analysis for general flags is substantially more complicated than for a single Grassmannian because $\operatorname{Rat}_{1, \ldots, 1}(\mathbb{F}(J))$ consists of more than one stratum and we must see how they fit together in the Rat space. In $\S 10$ we use the main results here, Lemma 9.2, (9.8), (9.15), which describe the explicit structure of the two strata for $\operatorname{Rat}_{1,1}\left(\mathbb{F}\left(j_{1}, j_{2} ; n\right)\right)$ and the attaching maps on the boundaries of their normal bundles. These have evident generalizations to the spaces $\operatorname{Rat}_{\overrightarrow{1}}(\mathbb{F}(J))$ and enable us, in principle, to determine the cohomology of these spaces as well.

The results of $\S 6$ show that $\operatorname{Rat}_{1,1}\left(\mathbb{F}\left(j_{1}, j_{2}\right)\right)$ is the union of two strata, $Y(1,0,1)$ and $Y(0,1,0)$. The first is open in $\operatorname{Rat}_{1,1}\left(\mathbb{F}\left(j_{1}, j_{2}\right)\right)$ and consists of points with representations

$$
\left[D_{1}, N_{1}\right]\left[\left(\begin{array}{cc}
I & C \\
0 & D_{2}
\end{array}\right),\left(\begin{array}{c}
0 \\
N_{2}
\end{array}\right)\right],
$$

where $\operatorname{det}\left(D_{1}\right)=z-A, \operatorname{det}\left(D_{2}\right)=z-B$. It is given as the total space of a fibration

$$
\begin{aligned}
Y(1,0,0) \simeq & \operatorname{Rat}_{1}\left(\mathbb{G}_{j_{1}, j_{2}-j-1}\right) \\
& \rightarrow Y(1,0,1) \rightarrow \operatorname{Rat}_{1}\left(\mathbb{G}_{j_{2}-j_{1}, n-j_{2}}\right) \simeq Y(0,0,1) .
\end{aligned}
$$


To describe this fibration we need to consider line bundles over $\mathbb{C P}^{m} \times \mathbb{C P}^{l}$. Any such line bundle is determined by its first Chern class, and will be written as $\xi_{m, l}^{a, b}$.

Here $a c_{1}+b d_{1}$ is the first Chern class of $\xi_{m, l}^{a, b}$ where $c_{1}$ and $d_{1}$ are the first Chern classes of the tautological line bundles over $\mathbb{C P}^{m}$ and $\mathbb{C P}^{l}$ respectively. If $a=0$ or $b=0$, we will sometimes abbreviate this bundle by $\xi_{l}^{b}$ or $\xi_{m}^{a}$, respectively.

If we have a vector bundle $\xi \rightarrow X$ where $\xi=\zeta \oplus \nu$ is the Whitney sum of two bundles, then the complement of the mutual 0 -sections is defined to be the set of points $(v, w) \in \zeta \oplus \nu, v \in \zeta, w \in \nu$ with neither $v$ nor $w$ on their respective 0 -sections.

Lemma 9.2. If $j_{2}-j_{1} \geq 2$, then $Y(1,0,1)$ is homeomorphic to the Cartesian product of $\mathbb{C}^{2}$ with the complement of the mutual 0-sections in the Whitney sum

$$
\left[\xi_{j_{1}-1, j_{2}-j_{1}-1}^{-1,1} \oplus \xi_{j_{1}-1, j_{2}-j_{1}-1}^{-1,-1} \oplus\left(j_{2}-j_{1}-2\right) \xi_{j_{1}-1}^{-1}\right] \oplus\left[\left(n-j_{2}\right) \xi_{j_{2}-j_{1}-1}^{-1}\right] .
$$

On the other hand if $j_{2}=j_{1}+1$, then $Y(1,0,1)$ is homeomorphic to the Cartesian product of $\mathbb{C}^{2}$ with the complement of the mutual 0-sections in

$$
\left[\xi_{j-1-1,0}^{-1,1}\right] \oplus\left[\left(n-j_{2}\right) \xi_{0}^{-1}\right] \cong\left[\xi_{j_{1}-1}^{-1}\right] \oplus\left[\mathbb{C}^{\left(n-j_{2}\right)}\right] .
$$

Proof. Every point of $Y(1,0,1)$ can be written as

$$
\left[D_{1}, N_{1}\right]\left[\left(\begin{array}{cc}
I & C \\
0 & D_{2}
\end{array}\right),\left(\begin{array}{c}
0 \\
N_{2}
\end{array}\right)\right]
$$

where

$$
\begin{aligned}
D_{1} & =\left(\begin{array}{ccc}
I_{r} & E & 0 \\
0 & z-A & 0 \\
0 & 0 & I_{j_{1}-r-1}
\end{array}\right), \quad N_{1}=\left(\begin{array}{c}
0_{r \times\left(j_{2}-j_{1}\right)} \\
\vec{w} \\
0_{\left(j_{1}-r-1\right) \times\left(j_{2}-j_{1}\right)}
\end{array}\right), \\
D_{2} & =\left(\begin{array}{ccc}
I_{s} & F & 0 \\
0 & z-B & 0 \\
0 & 0 & I
\end{array}\right) .
\end{aligned}
$$

Here $E$ and $F$ are column vectors, and $\vec{w}$ is a row vector. Recall that $D_{1}$ and $D_{2}$ give local coordinates for the base $\mathbb{C P}^{\left(j_{1}-1\right)}$ and $\mathbb{C P}^{\left(j_{2}-j_{1}-1\right)}$ respectively, whereas the $N_{1}$ and $N_{2}$ give local coordinates for the fibers of the respective bundles. The roots of the linear terms, $A$ and $B$, fill out a copy of $\mathbb{C}^{2}$. Next, the constraint condition, $((4.4)$ and (4.5)) that insures $(9.5)$ represents a based map, $\lim _{z \rightarrow \infty}\left(C+D_{1}^{-1} N_{1} D_{2}\right)=0$ uniquely determines the one nonzero column of $C$ in terms of $w_{s+1}$ and $E$. 
Theorem 8.2 and Corollary 8.6 show that to understand the interaction of these components it suffices to replace $D_{2}$ by

$$
D_{2}^{\prime}=\left(\begin{array}{cccc}
z-B & 0 & \cdots & 0 \\
h_{2} & 1 & \cdots & 0 \\
\vdots & \vdots & \ddots & \vdots \\
h_{j_{2}-j_{1}} & 0 & \cdots & 1
\end{array}\right)
$$

and track the effect of reducing this new point to normal form. There exists a $U \in \mathrm{GL}_{j_{2}}(\mathbb{C}[z])$ such that

$$
\left[\left(\begin{array}{cc}
I & C^{\prime} \\
0 & D_{2}^{\prime}
\end{array}\right),\left(\begin{array}{c}
0 \\
N_{2}^{\prime}
\end{array}\right)\right] \mapsto\left[U\left(\begin{array}{cc}
I & C^{\prime} \\
0 & D_{2}^{\prime}
\end{array}\right), U\left(\begin{array}{c}
0 \\
N_{2}^{\prime}
\end{array}\right)\right]=\left[\begin{array}{ccc}
I & C & 0 \\
0 & D_{2} & N_{2}
\end{array}\right]
$$

and

$$
\left[D_{1}, N_{1}\right] \mapsto\left[D_{1} U^{-1}, N_{1} U^{-1}\right]
$$

Indeed, explicitly constructing $U$ will show that it has the form

$$
U=\left(\begin{array}{cc}
I & J \\
0 & U_{2}
\end{array}\right)
$$

where $U_{2} \in \mathrm{GL}_{j_{2}-j_{1}}(\mathbb{C}[z])$. This implies that $D_{1} U^{-1}=D_{1}$ and, thus, that the twisting of the two normal forms involves only the fibre coordinates.

Recall from $\S 8$ that we are actually using the following embedding

$$
\mathbb{C P}^{l} \subset \mathrm{GL}_{j_{2}-j_{1}}(\mathbb{C}[z]) \backslash\left\{M_{j_{2}-j_{1}}(1)\right\},
$$

where $\left\{M_{j_{2}-j_{1}}(i)\right\} \subset \mathrm{GL}_{j_{2}-j_{1}}(\mathbb{C}(z)) \cap M_{j_{2}-j_{1}}(\mathbb{C}[z])$ is the set of elements with determinant of degree exactly $i$ as a polynomial in $z$.

$\mathbb{C P}^{j_{2}-j_{1}-1}$ has $j_{2}-j_{1}$ coordinate neighborhoods $U_{i}=\mathbb{C}^{j_{2}-j_{1}-1}$ where the $j$ th neighborhood has coordinates $\left(v_{1}^{j}, \cdots, v_{j_{2}-j_{1}-1}^{j}\right)$ and $U_{1} \cap U_{j} \subset$ $U_{1}$ are those points with $v_{j}^{1} \neq 0$. In this region the transition function is

$$
\left(v_{1}^{1}, \cdots, v_{j_{2}-j_{1}-1}^{1}\right) \mapsto\left(\frac{1}{v_{j}^{1}}, \frac{v_{1}^{1}}{v_{j}^{1}}, \cdots, \widehat{v_{j}^{1}}, \cdots, \frac{v_{j_{2}-j_{1}-1}^{1}}{v_{j}^{1}}\right) .
$$

The embedding is then given by

$$
U_{1} \mapsto\left(\begin{array}{cccc}
-z & 0 & \cdots & 0 \\
v_{1}^{1} & -1 & \cdots & 0 \\
\vdots & \vdots & \ddots & \vdots \\
v_{j_{2}-j_{1}-1}^{1} & 0 & \cdots & -1
\end{array}\right) \cdots U_{j_{2}-j_{1}} \mapsto\left(\begin{array}{ccccc}
-1 & 0 & \cdots & 0 & v_{1}^{j_{2}-j_{1}} \\
\vdots & \vdots & \ddots & \vdots & \vdots \\
0 & 0 & \cdots & -1 & v_{l}^{j_{2}-j_{1}} \\
0 & 0 & \cdots & 0 & -z
\end{array}\right) .
$$


Furthermore, the transition matrices in $\mathrm{GL}_{j_{2}-j_{1}}(\mathbb{C}[z])$ between $U_{1}$ and $U_{j}$ are given by the $T_{j}^{-1}$ 's where $T_{j}$ is the composition

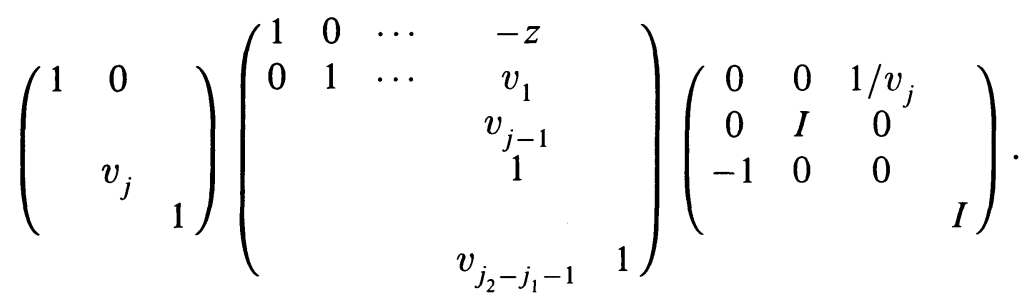

Consequently, in (9.7), the submatrix $U_{2}$ in the matrix $U$ for this transition is $T_{j}^{-1}$. This implies, under $U^{-1}$, that $\vec{w} \mapsto-J T_{j}^{-1}+\vec{w} T_{j}$. Explicitly, when we apply each $T_{j}^{-1}$ to the fiber coordinates $\vec{w} \in N_{1}$ we have

$(\vec{w}) T_{j}=\left(-v_{1} w_{2}-\cdots-v_{r} w_{j_{2}-j_{1}}, w_{2}, \cdots, w_{j-1}, \frac{w_{1}}{v_{j}}, w_{j+1}, \cdots, w_{j_{2}-j_{1}}\right)$.

In the rather degenerate case, when $j_{2}=j_{1}+1,(9.4)$ follows from the facts that $\mathbb{C P}^{j_{2}-j_{1}-1}$ is just a point and $\vec{w}$ is just the scalar $w_{1}$. Also, if $j_{2}=j_{1}+2$, then $(9.8)$ is just $\left(-v_{1} w_{2}, w_{1} / v_{2}\right)$ and we recover $(9.3)$ in this case.

For the remainder of the proof we assume $j_{2}-j_{1}>2$. On a basis $e_{1}^{1}, \cdots, e_{j_{2}-j_{1}-1}^{1}$ the transformation in (9.8) takes the form

$$
e_{s}^{1} \mapsto \begin{cases}\left(1 / v_{j}\right) e_{j}^{j} & \text { when } s=1, \\ -v_{j} e_{1}^{j} & \text { when } s=j, \\ -v_{s} e_{1}^{j}+e_{s}^{j} & \text { otherwise. }\end{cases}
$$

This means that the bundle splits as $\xi_{j_{2}-j_{1}-1}^{-1} \oplus W$ where $W$ has the basis $\left\{e_{k}^{j} \mid k \neq j\right\}$ over $U_{j}$ and transition function

$$
\begin{aligned}
& e_{j}^{1} \mapsto-v_{j}^{1} e_{1}^{j}, \\
& e_{s}^{1} \mapsto-v_{s}^{1} e_{1}^{j}+e_{s}^{j}, \quad s \neq j .
\end{aligned}
$$

Computing further we find that the $j$ th transition matrix for $W$ is given 
by

$$
\left(\begin{array}{cccc}
-v_{1} & 1 & \cdots & 0 \\
\vdots & \vdots & \ddots & \vdots \\
-v_{j} & 0 & \cdots & 0 \\
\vdots & \vdots & \ddots & \vdots \\
-v_{j_{2}-j_{1}-1} & 0 & \cdots & 1
\end{array}\right) .
$$

To understand this bundle it is easier to consider the adjoint bundle $W^{*}$ with transition matrices given by the transpose inverses of the transition matrices for $W$. Explicitly they are

$$
\left(\begin{array}{ccc}
-v_{1} / v_{j} & -1 / v_{j} & -v_{j_{2}-j_{1}-1} / v_{j} \\
1 & 0 & 0 \\
0 & 0 & 1
\end{array}\right)
$$

so

$$
e_{s}^{1} \mapsto \begin{cases}e_{s}^{j} & \text { when } s<j \text { and } s \geq 2, \\ e_{s-1}^{j} & \text { when } s>j .\end{cases}
$$

It follows that the $j_{2}-j_{1}-2$ local sections defined by $\left\{e_{s}^{1}\right\}$ for $s \neq 1$ and $s \neq j$ in the $j=1$ coordinate chart extend to global sections and span a trivial bundle $\left(j_{2}-j_{1}-2\right) \varepsilon \subset W^{*}$. Therefore, $W^{*}=\left(j_{2}-j_{1}-2\right) \varepsilon \oplus \xi_{j_{2}-j_{1}-1}^{i}$ for some $i$. On the other hand the determinant bundle of $W^{*}$ is $\xi_{j_{2}-j_{1}-1}$ and hence we have proved that $W^{*}=(r-1) \varepsilon \oplus \xi_{j_{2}-j_{1}-1}$. Therefore, $C\left(W^{*}\right)$, the total Chern class of $W^{*}$, is $1-b$. But, if $c_{i}$ is the $i$ th Chern class of the original bundle, then the $i$ th Chern class of the adjoint bundle is $-1^{i} c_{i}$ and so $C(W)=1+b$. Therefore, as our original bundle has fiber dimension greater than that of the base, it must be isomorphic, as a complex vector bundle, to $\left(j_{2}-j_{1}-2\right) \varepsilon \oplus \xi_{j_{2}-j_{1}-1}^{1} \oplus \xi_{j_{2}-j_{1}-1}^{-1}$ with total Chern class $(1+b)(1-b)=1-b^{2}$. q.e.d.

We note in passing that the splitting in (9.3) is as a complex vector bundle, but there is no reason to assume this splitting preserves the holomorphic structure of $Y(1,0,1)$. Also, the holomorphic vector bundle $W$ occuring in the proof of Lemma 9.2 above will play a critical role in what follows.

We will need the following explicit model for the homotopy type of $Y(1,0,1)$ which is direct from Lemmas 8.11 and 9.2. 
Corollary 9.9. $Y(1,0,1)$ is homotopy equivalent to

$$
\mathbb{C}^{2} \times\left(S^{2 j_{1}-1} \times S^{2\left(j_{2}-j_{1}\right)-1} \times S^{2\left(j_{2}-j_{1}\right)-1} \times S^{2\left(n-j_{2}\right)-1}\right) /\left(S^{1} \times S^{1}\right),
$$

where the $S^{1} \times S^{1}$ action is given by

$$
\begin{gathered}
z(\vec{a}, \vec{b}, \vec{c}, \vec{d})=\left(z \vec{a}, z^{-1} \vec{b}, \vec{c}, \vec{d}\right), \\
w\left(\vec{a}, b_{1}, b_{2}, b_{3}, \cdots, b_{j_{2}-j_{1}}, \vec{c}, \vec{d}\right) \\
=\left(\vec{a}, w b_{1}, w^{-1} b_{2}, b_{3}, \cdots, b_{j_{2}-j_{1}}, w \vec{c}, w^{-1} \vec{d}\right) .
\end{gathered}
$$

Remark 9.11. Lemma 9.2 generalizes easily to describe the geometry of the corresponding generic set $Y(1,0,1,0,0,1)$ in $\operatorname{Rat}_{1,1,1}\left(\mathbb{F}\left(j_{1}, j_{2}, j_{3}\right)\right)$. Indeed, this is the complement of the 0 -sections in the sum

$$
\begin{aligned}
& {\left[\xi_{j_{1}-1, j_{2}-j_{1}-1}^{-1,1,0} \oplus \xi_{j_{1}-1, j_{2}-j_{1}-1}^{-1,-1,0} \oplus\left(j_{2}-j_{1}-2\right) \xi_{j_{1}-1}^{-1,0,0}\right]} \\
& \quad \oplus\left[\xi_{j_{2}-j_{1}-1, j_{3}-j_{2}-1}^{0,-1,1} \oplus \xi_{j_{2}-j_{1}-1, j_{3}-j_{2}-1}^{0,-1,-1} \oplus\left(j_{3}-j_{2}-2\right) \xi_{j_{2}-j_{1}-1}^{0,-1,0}\right] \\
& \quad \oplus\left[\left(n-j_{3}\right) \xi_{j_{3}-j_{2}-1}^{0,0,-1}\right] .
\end{aligned}
$$

Here we have assumed that each $j_{i+1}-j_{i}-1>0$ with the obvious modification required in the complementary cases. Similar constructions for arbitrary $m$ give all of the strata in $\operatorname{Rat}_{(1, \cdots, 1)}\left(\mathbb{F}\left(j_{1}, \cdots, j_{m}\right)\right)$.

We now turn our attention to the second stratum, $Y(0,1,0)$, and the way in which it attaches to $Y(1,0,1), Y(0,1,0)$ consists of all matrices of the form

$$
\left[I,\left(\begin{array}{ccccc}
0 & \ldots & 0 & \cdots & 0 \\
v_{1} & \cdots & v_{l} & \cdots & v_{j_{2}-j_{1}} \\
0 & \cdots & 0 & \cdots & 0
\end{array}\right)\right]\left[\left(\begin{array}{cc}
D_{2}^{\prime} & 0 \\
0 & I
\end{array}\right),\left(\begin{array}{c}
N_{2}^{\prime} \\
0
\end{array}\right)\right]
$$

where

$$
D_{2}^{\prime}=\left(\begin{array}{ccc}
I_{l-1} & T_{1} & 0 \\
0 & z-B & 0 \\
0 & T_{2} & I
\end{array}\right)
$$

and the only nonzero row in $N_{2}^{\prime}$ is the $l$ th which is given by the vector $\bar{w}$. After multiplying these pairs to get a pair representing a point of $\operatorname{Rat}_{1}\left(\mathbb{G}_{j_{1}, n-j_{2}}\right)$ we see that these quadruples are equivalent to pairs

$$
\left[D_{2}^{\prime},\left(\begin{array}{cccccccc}
0 & \ldots & 0 & \ldots & 0 & 0 & \ldots & 0 \\
v_{1} & \ldots & v_{l} & \ldots & v_{j_{2}-j_{1}} & w_{1} & \ldots & w_{n-j_{2}} \\
0 & \ldots & 0 & \ldots & 0 & 0 & \ldots & 0
\end{array}\right)\right]
$$

which give the points in the subspace of $\operatorname{Rat}_{1}\left(\mathbb{G}_{j_{1}, n-j_{1}}\right)$ determined by the constraint $\sum\left|w_{i}\right| \neq 0$. Consequently $Y(0,1,0)$, which is homotopy 
equivalent to

$$
\left(S^{2 j_{1}-1} \times S^{2\left(n-j_{2}\right)-1}\right) / S^{1}
$$

is the total space of the bundle $\rho^{*}\left(\left(j_{2}-j_{1}\right) \xi_{j_{1}-1}^{-1}\right)$ over $\operatorname{Rat}_{1}\left(\mathbb{G}_{j_{1}, n-j_{2}}\right)$ pulled back from the projection $\rho$ onto $\mathbb{C P}^{j_{1}-1}$. The fiber coordinates are $\left(v_{1}, \cdots, v_{j_{2}-j_{1}}\right)$ and there is one more free coordinate, $B$.

Proposition 9.14. The normal bundle to $Y(0,1,0)$ in $\operatorname{Rat}_{1,1}\left(\mathbb{F}\left(j_{1}, j_{2}\right)\right)$, $\nu(Y(0,1,0))$, is the pullback of $\left(j_{2}-j_{1}\right) \xi_{j_{1}}$ under the projection

$$
Y(0,1,0) \rightarrow \operatorname{Rat}_{1,1}\left(\mathbb{F}\left(j_{1}, j_{2}\right)\right) \rightarrow \operatorname{Rat}_{1}\left(\mathbb{F}\left(j_{2}\right)\right) \rightarrow \mathbb{C P}^{j_{2}-1} .
$$

Proof. The map $\operatorname{Rat}_{1,1}\left(\mathbb{F}\left(j_{1}, j_{2}\right)\right) \rightarrow \mathbb{C P}^{j_{2}-1}$ is holomorphic and transverse regular on $\mathbb{C P}^{j_{1}-1}$ with inverse image $Y(0,1,0)$. Consequently, the normal bundle is the pullback under this map of the normal bundle of $\mathbb{C P}^{j_{1}-1}$ in $\mathbb{C P}^{j_{2}-1}$, which is $\left(j_{2}-j_{1}\right) \xi_{j_{1}-1}$. q.e.d.

The normal sphere bundle is thus homotopic to the space

$$
V\left(j_{1}, j_{2}, n\right)=\left(S^{2\left(j_{2}-j_{1}\right)-1} \times S^{2 j_{1}-1} \times S^{2\left(n-j_{2}\right)-1}\right) / S^{1},
$$

where the $S^{1}$-action is given by

$$
z(\vec{A}, \vec{B}, \vec{C})=\left(z \vec{A}, z \vec{B}, z^{-1} \vec{C}\right) .
$$

In particular, up to homotopy type $\operatorname{Rat}_{1,1}\left(\mathbb{F}\left(j_{1}, j_{2}\right)\right)$ is given as the double mapping cylinder associated to the maps

$$
\left(S^{2 j_{1}-1} \times S^{2\left(n-j_{2}\right)-1}\right) / S^{1} \stackrel{p}{\longleftarrow} V\left(j_{1}, j_{2}, n\right) \stackrel{\phi}{\longrightarrow} Y(1,0,1),
$$

and the major difficulty remaining in understanding $\operatorname{Rat}_{1,1}\left(\mathbb{F}\left(j_{1}, j_{2}\right)\right)$ is the determination of the map $\phi$.

Recall that

$$
Y(1,0,1) \simeq\left(S^{2 j_{1}-1} \times S^{2\left(j_{2}-j_{1}\right)-1} \times S^{2\left(j_{2}-j_{1}\right)-1} \times S^{2\left(n-j_{2}\right)-1}\right) /\left(S^{1} \times S^{1}\right),
$$

which, in turn, is the homotopy type of the sphere bundle in the holomorphic vector bundle

$$
\mathbb{C}^{j_{2}-j_{1}} \rightarrow \xi_{j_{1}-1, j_{2}-j_{1}-1}^{-1,1} \oplus W \rightarrow \mathbb{C P}^{j_{1}-1} \times\left\{\left(S^{2\left(j_{2}-j_{1}\right)-1} \times S^{2\left(n-j_{2}\right)-1}\right) / S^{1}\right\}
$$

We have

Proposition 9.16. The space $V\left(j_{1}, j_{2}, n\right)$ is homeomorphic to the circle bundle in the fibering $\xi_{j_{1}-1, j_{2}-j_{1}-1}^{-1,1} \rightarrow \mathbb{C P}^{j_{1}-1} \times \operatorname{Rat}_{1}\left(\mathbb{F}\left(j_{2}-j_{1}\right)\right)$, and the 
map $\phi$ is homotopic to the natural inclusion

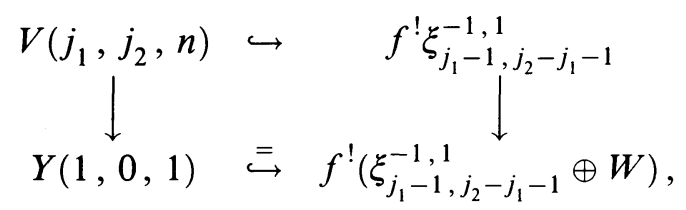

where $f: \mathbb{C P}^{j_{1}-1} \times \operatorname{Rat}_{1}\left(\mathbb{G}_{j_{2}-j_{1}, n-j_{2}}\right) \rightarrow \mathbb{C P}^{j_{1}-1} \times \mathbb{C P}^{j_{2}-j_{1}-1}$ is the projection.

Proof. Recall from $\S 5$ that the normal bundle calculations in terms of normal forms proceeds by perturbing an element of the form in (9.12) by placing a nonzero vector $\vec{\varepsilon}=\left(\varepsilon_{1}, \cdots, \varepsilon_{\left(j_{2}-j_{1}\right)}\right)$ in the lth column of the $D(2)$ matrix (in the notation of (9.12) the vector $\vec{\varepsilon}$ is placed in the lth column of the zero matrix directly below the $D_{2}^{\prime}$ submatrix) and then using a unimodular matrix $U$ to bring

$$
\left[I,\left(\begin{array}{ccc}
0 & \cdots & 0 \\
v_{1} & \cdots & v_{j_{2}-j_{1}} \\
0 & \cdots &
\end{array}\right)\right] U^{-1} U\left[\left(\begin{array}{cc}
D_{2}^{\prime} & 0 \\
\vec{\varepsilon} & I
\end{array}\right),\left(\begin{array}{c}
N_{2}^{\prime} \\
0
\end{array}\right)\right]
$$

into normal form. Expanding out, $U^{-1}$ has the form

$$
\left(\begin{array}{ccccc}
I & C & 0 & 0 & 0 \\
0 & z-B & 0 & -1 / \varepsilon_{l} & 0 \\
0 & E_{1} & I & 0 & 0 \\
0 & \varepsilon_{l} & 0 & 0 & 0 \\
0 & E_{2} & 0 & 0 & I
\end{array}\right)
$$

and we can rewrite $(9.17)$ as

$$
\left[D_{1}, N_{1}\right]\left[\left(\begin{array}{cc}
I & C \\
0 & D_{2}
\end{array}\right),\left(\begin{array}{c}
0 \\
N_{2}
\end{array}\right)\right]
$$

where

$$
D_{1}=\left(\begin{array}{ccc}
I_{l-1} & T_{1} & 0 \\
0 & z-B+\vec{\varepsilon} \cdot \vec{v} & 0 \\
0 & T_{2} & I
\end{array}\right) \quad \text { and } \quad D_{2}=\left(\begin{array}{ccc}
I_{s-1} & E_{1, s} & 0 \\
0 & z-B & 0 \\
0 & E_{2, s} & I
\end{array}\right) .
$$

Here

(a) the one nonzero row of $N_{1}$ is obtained from $\vec{v}$ by replacing $v_{l}$ by $-1 / \varepsilon$, and leaving the remaining entries alone,

(b) the one nonzero row of $N_{2}$ is $-\varepsilon_{l} \vec{w}$; that is, $-\varepsilon_{l}$ times the original nonzero row of $N_{2}^{\prime}$ in 9.12 ,

(c) the one nonzero column of the $C$ matrix, $C_{s}$, is the vector $\left(1 / \varepsilon_{l}\right)\left(-T_{1}, 1,-T_{2}\right)^{t}$,

(d) $E_{1, l}=\left(-1 / \varepsilon_{l}\right)\left(\varepsilon_{1}, \cdots, \varepsilon_{l-1}\right)^{t}, E_{2, l}=\left(-1 / \varepsilon_{l}\right)\left(\varepsilon_{l+1}, \cdots, \varepsilon_{j_{2}-j_{1}}\right)^{t}$. 
As has been observed the $\vec{v}$ coordinates and $B$ are free to vary linearly over $\mathbb{C}^{j_{2}-j_{1}+1}$ and hence we can set them all to zero, so $N_{1}$ has the form $\left(-1 / \varepsilon_{l}\right) e_{l}$. In view of the discussion in the proof of Lemma 9.2 (especially the remarks after (9.7)), which describes how $\xi_{j_{1}-1, j_{2}-j_{1}-1}^{-1,1}$ splits off, we see that this image is exactly the circle bundle in $\xi_{j_{1}-1, j_{2}-1-1}^{-1,1}$.

It remains to observe the homeomorphism. We can describe the total space of this circle bundle as the quotient under the action of $S^{1} \times S^{1}$ on

$$
\begin{gathered}
S^{2 j_{1}-1} \times S^{1} \times S^{2\left(j_{2}-j_{1}\right)-1} \times S^{2\left(n-j_{2}\right)-1}, \\
(z, \zeta)(\vec{A}, \vec{B}, \vec{C}, \vec{D})=\left(z \vec{A}, z^{-1} \zeta \vec{B}, \zeta \vec{C}, \zeta^{-1} \vec{D}\right) .
\end{gathered}
$$

Note that the diagonal copy of $S^{1} \subset S^{1} \times S^{1}$, acts trivially on the $S^{1}$. Thus the quotient by this action is the product $S^{1} \times$ (sphere bundle of normal bundle). Now acting by the second $S^{1}$ gives the result since the action is free on the extra $S^{1}$.

Remark 9.18. From the perspective of holomorphic bundles we see that Proposition 9.16 is forced. Indeed, note that the map to the two extremes $\left(S^{2 j_{1}-1} \times S^{2\left(j_{2}-j_{1}\right)-1}\right) / S^{1} \rightarrow \mathbb{C P}^{j_{1}-1} \times \mathbb{C P}^{j_{2}-j_{1}-1}$ in cohomology takes $b_{i}$ to the generator for both $i=1$ and 2 . Since this map factors through the complement of the 0 -section in the bundle $\xi_{j_{1}-1, j_{2}-j_{1}-1}^{-1,1} \oplus W \rightarrow \mathbb{C P}^{j_{1}-1} \times \mathbb{C P}^{j_{2}-j_{1}-1}$, the induced bundle over $\left(S^{2 j_{1}-1} \times S^{2\left(j_{2}-j_{1}\right)-1}\right) / S^{1}$ has an everywhere nonzero holomorphic section. But, by restricting to a diagonally embedded $\mathbb{C P}^{1}$, we see that, if indeed $W$ has any holomorphic sections, they must always have zeros. On the other hand, the bundle induced from $\xi^{-1,1}$ is the trivial bundle so the section must be nontrivial in the complement of the zero section in $\xi^{-1,1}$. Thus, it deforms to this section.

Remark 9.19. This discussion extends directly to the general case, and with the obvious modifications, Proposition 9.14 describes the normal bundle of the general stratum in any larger stratum while (9.15) describes the homotopy type of the attaching of the normal sphere bundle to this stratum into one of the larger strata.

\section{The homology of $\operatorname{Rat}_{(1, \cdots, 1)}(\mathbb{F}(J))$}

In this section we study the cohomology of the strata in Rat ${ }_{1, \ldots, 1}(\mathbb{F}(J))$ and the map $\phi^{*}$ associated to the map $\phi$ in (9.15). In certain cases the information we obtain is sufficient to entirely determine $H^{*}\left(\operatorname{Rat}_{1, \ldots, 1}(\mathbb{F}(J))\right)$, and, in all cases, it provides a complete algorithm for doing the calculation. 
We begin with some basic spaces and their cohomology rings. Denote by $S(k, l+k)$ the $S^{2 l-1}$ sphere bundle of the holomorphic vector bundle

$$
\mathbb{C}^{l} \rightarrow \xi_{k-1, l-1}^{-1,1} \oplus W \rightarrow \mathbb{C P}^{k-1} \times \mathbb{C P}^{l-1} \text {. }
$$

Consider the Gysin sequence of the bundle $S(k, l+k) \rightarrow \mathbb{C P}^{k-1} \times \mathbb{C P}^{l-1}$,

$$
\begin{aligned}
\cdots \rightarrow H^{*-1} & (S(k, l+k)) \stackrel{\delta}{\rightarrow} H^{*}\left(T\left(\xi_{K-1, l-1}^{-1,1} \oplus W\right)\right) \\
& \stackrel{\cup \chi(V)}{\longrightarrow} H^{*}\left(\mathbb{C P}^{k-1} \times \mathbb{C P}^{l-1}\right) \rightarrow H^{*}(S(k, l+k)) \stackrel{\delta}{\longrightarrow} \cdots
\end{aligned}
$$

where $T(V)$ is the Thom complex with Thom class $U_{V}, \chi(V)$ is the Euler class of the bundle $V$, and $\cup \chi(V)$ is the map $a \cup U_{V} \mapsto a \cup \chi(V)$. Recall here that if $V$ is a complex vector bundle with fiber $\mathbb{C}^{n}$, then $\chi(V)=c_{n}(V)$, the $n$th Chern class.

It follows that the image of $H^{*}\left(\mathbb{C P}^{k-1} \times \mathbb{C P}^{l-1}\right) \rightarrow H^{*}(S(k, l+k))$ is

$$
\mathscr{A}(k, l)=\mathbb{Z}\left[b_{1}, b_{2}\right] /\left(b_{1}^{k}=b_{2}^{l}=b_{1}^{l-2}\left(b_{1}^{2}-b_{2}^{2}\right)=0\right) .
$$

If $k \leq l-2$, then (10.1) becomes especially simple and we have

Proposition 10.3. If $k \leq l-2$, then

$$
H^{*}(S(k, l+k))=\mathscr{A}(k, l)\left(1, e_{2 l-1}\right)=\frac{\mathbb{Z}\left[b_{1}, b_{2}\right]}{b_{1}^{k}=b_{2}^{l}=0}\left(1, e_{2 l-1}\right)
$$

where $e_{2 l-1}$ is dual to the spherical class of the fiber.

Therefore, in what follows we assume $k>l-2$. To set notation let $a=\max (0,2 l-2-k)$ so that

$$
l-a= \begin{cases}l & \text { when } k \geq 2 l-2, \\ k-l+2 & \text { if } l-2<k<2 l-2 .\end{cases}
$$

Also, let $\varepsilon$ be the parity of $l$; that is,

$$
\varepsilon= \begin{cases}1 & \text { when } l \text { odd } \\ 0 & \text { when } l \text { even }\end{cases}
$$

Next, set

$$
C(l)=\mathbb{Z}\left[b_{1}, b_{2}\right] /\left(b_{2}^{l}=b_{l}^{l-2}\left(b_{1}^{2}-b_{2}^{2}\right)=0\right),
$$

and

$$
p_{i}=b_{1}^{i}+b_{1}^{i-2} b_{2}^{2}+b_{1}^{i-4} b_{2}^{4}+\cdots+b_{2}^{i}
$$

for $i$ even. Finally, let $\mathscr{M}_{a, l}$ be the module over $C(l)$ on two generators $s, h$, with $\operatorname{dim}(h)=0$ and $\operatorname{dim}(s)=2(a+\varepsilon-2)$, specified by relations

$$
\begin{aligned}
b_{1}^{l-2} h & =0, \\
b_{2}^{\varepsilon} b_{1}^{a} h & =\left(b_{1}^{2}-b_{2}^{2}\right) s, \\
b_{2}^{\varepsilon} p_{l-2-\varepsilon} h & =b_{1}^{l-a} s .
\end{aligned}
$$


Notice that, in certain cases, the dimension of $s$ is negative. With this notation we have

Proposition 10.5. Assume $l, k \geq 3$.

(a) If $k=2 l-2$ with $l$ odd, then

$$
H^{*}(S(k, l+k))=\mathscr{A}(k, l) \oplus \mathscr{W},
$$

where $\mathscr{W}$ has generators $w=p_{l-1} e, v=b_{1} b_{2} p_{l-3} e$, so $\operatorname{dim}(w)=$ $\operatorname{dim}(v)=4 l-3$, and the relations are $w \cdot v=0, b_{1}^{l-2}\left(b_{1} w-b_{2} v\right)=0$, $b_{2} w=b_{1} v$.

(b) If $l-2<k<2 l-2$ or $l$ is even, then

$$
H^{*}(S(k, l+k))=\mathscr{A}(k, l) \oplus \Sigma^{2 k+3}\left(\mathscr{M}_{a, l}\right) .
$$

Moreover, in the Gysin sequence (10.1) we have

$$
\begin{aligned}
& \delta\left(\Sigma^{2 k+3} h\right)=b_{1}^{k-l+2} U, \\
& \delta\left(\Sigma^{2 k+3} s\right)=b_{2}^{\varepsilon} b_{1}^{d}\left(\frac{b_{1}^{l-\varepsilon}-b_{2}^{l-\varepsilon}}{b_{1}^{2}-b_{2}^{2}}\right) U,
\end{aligned}
$$

where $U$ is the Thom class, and $d=\max (0, k-2 l+2)$.

(c) If $k>2 l-2$ and $l$ is odd, then

$$
H^{*}(S(k, l+k))=\mathscr{A}(k, l) \oplus \mathscr{N}(l),
$$

where $\mathcal{N}(l)$ has generator $s$ of dimension $2 k-1$ with relations

$$
b_{1}^{l-2}\left(b_{1}^{2}-b_{2}^{2}\right) s=b_{1}^{k} s=0, \quad \delta(s)=b_{1}^{d-1}\left(\frac{b_{1}^{l+1}-b_{2}^{l+1}}{b_{1}^{2}-b_{2}^{2}}\right) U .
$$

Proof. Fix $l$ and let $k$ increase, starting at $l-1$. The Euler class $\chi(S(k, l+k))=b_{1}^{l-2}\left(b_{1}^{2}-b_{2}^{2}\right)$ and so, in this first case, equals $-b_{1}^{l-2} b_{2}^{2}$. In particular, the kernel is clearly generated by $b_{1} U$ and $b_{2}^{l-2} U$. Now increase $k$ by 1 . The Euler class is unchanged, and $b_{1} U$ now acts like $U$ did previously, giving kernel classes $b_{1}^{2} U$ and $b_{1} b_{2}^{l-2} U$. Moreover, the previous kernel class $b_{2}^{l-2} U$ remains in the kernel. Keep extending in this way by increasing $k$. Notice that each time we go up by 2 in $k$, the multiplier on $b_{2}^{l-2} U$ gets a term added to it, first $b_{1}^{2} b_{2}^{l-4}$, then $b_{1}^{4} b_{2}^{l-6}$ and so on until the term $l-2 r$ becomes $\varepsilon$. At this stage, the image of this class, under multiplication by the Euler class, is $b_{1}^{2 l-2-\varepsilon} b_{2}^{\varepsilon}$, which is a class that has not previously been hit.

Now continue to increase $k$. If $l$ is even then nothing more complex happens. From this point on the generating classes $\left(b_{1}^{d} p_{l-2} e\right)$ and 
$b_{2}\left(b_{1}^{d} p_{l-2} e\right)$ hit $b_{1}^{d+2 l-2}=b_{1}^{k-1}$ and $b_{2} b_{1}^{k-1}$, respectively, so that the new generating classes are $b_{1}^{d+1} p_{l-2} e$ and $b_{1}^{k-l+2} e$. This establishes part (b).

However, if $l$ is odd, then we have to be more careful. In case $k=2 l-2$ we see that $b_{2} p_{l-3} e \mapsto b_{1}^{k-1} b_{2}$, so $b_{2}^{2} p_{l-3} e$ and $b_{1} b_{2} p_{L-3} e$ survive to become generators. In this case we get the module in part (a). When $k=2 l-1$, then $b_{1} b_{2} p_{l-3} e \mapsto b_{2} b_{1}^{k-1}$ but the class $\left(b_{2}^{2} p_{l-3}+b_{1}^{l-1}\right) e$ survives. Hence, besides $h$, the generators are $b_{1}^{2} b_{2} p_{l-3} e$ and $p_{l-1} e$. Next, a direct calculation shows that this module does not change as $k$ increases further, even though the dimensions of the generators augment by 2 each time. This establishes part (c). q.e.d.

Finally, the cases not covered by Propositions 10.3 or 10.5 , when either $l$ or $k$ is less than 3 , are trivial and left to the reader.

These $S(k, l+k)$ spaces are the first stages of inductive building blocks for the strata $Y(1,0,1), Y(1,0,1,0,0,1)$, etc. For example, $Y(1,0,1)$ has the homotopy type of the sphere bundle in the fibration

$$
\mathbb{C}^{n-j_{2}} \rightarrow E \rightarrow S\left(j_{1}, j_{2}\right)
$$

with total Chern class $\left(1-b_{2}\right)^{n-j_{2}}$. To build $Y(1,0,1,0,0,1)$ we first construct the sphere bundle of the fibration

$$
\mathbb{C}^{j_{3}-j_{2}} \rightarrow E \rightarrow S\left(j_{1}, j_{2}\right) \times \mathbb{C P}^{j_{3}-j_{2}-1}
$$

with Chern class $\left(1-b_{2}\right)^{j_{3}-j_{2}-2}\left(1-b_{2}-b_{3}\right)\left(1-b_{2}+b_{3}\right)$. If we denote the total space here by $S\left(j_{1}, j_{2}, j_{3}\right)$, then we obtain $Y(1,0,1,0,0,1)$ as a homotopy type of the sphere bundle in the fibering

$$
\mathbb{C}^{n-j_{3}} \rightarrow E \rightarrow S\left(j_{1}, j_{2}, j_{3}\right)
$$

with total Chern class $\left(1-b_{3}\right)^{n-j_{3}}$. Moreover, the higher strata are obtained by repeating these constructions. Thus, in order to understand the next level of strata, we need to know $H^{*}\left(S\left(j_{1}, \cdots, j_{r}\right)\right)$ as a module over $\mathbb{Z}\left[b_{r}\right]$ since each Gysin sequence only involves this data.

It is easy to verify that at each stage the cohomology is torsion free, and this puts a severe restriction on the modules over $\mathbb{Z}\left[b_{r}\right]$ which can arise: they are all direct sums of cyclic modules, $\mathbb{Z}\left[b_{r}\right] /\left(b_{r}^{s}\right) e$.

In particular, for $S(k, l+k)$ we have

Corollary 10.8. Suppose $k>l-2$; then $H^{*}(S(k, l+k))$, as a module over $\mathbb{Z}\left[b_{2}\right]$, has the form

$$
\begin{aligned}
& \mathbb{Z}\left[b_{2}\right] /\left(b_{2}^{l}\right)\left\{1, b_{1}, \cdots, b_{1}^{l-3}, h, b_{1} h, \cdots, b_{1}^{l-1} h\right\} \\
& \oplus \mathbb{Z}\left[b_{2}\right] /\left(b_{2}^{\alpha}\right) b_{1}^{l-2} \oplus \mathbb{Z}\left[b_{2}\right] /\left(b_{2}^{\beta}\right) b_{1}^{l-1} \oplus \mathbb{Z}\left[b_{2}\right] /\left(b_{2}^{l-\alpha}\right) s \oplus \mathbb{Z}\left[b_{2}\right]\left(b_{2}^{l-\beta}\right) s^{\prime}
\end{aligned}
$$


Here $\alpha=\min \left(l, 2\left[\frac{k-l+1}{2}\right]\right)$ and $\beta=\min \left(l-\varepsilon, 2\left[\frac{k-l}{2}\right]\right)$ provided that $k \neq 2 l-2$ or $l$ is even. In the case $l$ odd and $k=2 l-2$, we have $\alpha=\beta=l-1$.

Proof. We have $b_{2}^{2} b_{1}^{l-2+t}=b_{1}^{l+t}$ for $t \geq 0$. Then the classes $b_{1}^{l-2}$ and $b_{1}^{l-1}$ generate two special modules, and the truncation is due to the fact that $b_{1}^{2 l-2}=0$ in $\mathscr{A}(k, l)$ for $l$ even, and $b_{2} b_{1}^{2 l-2}=b_{1}^{2 l-1}=0$ for $l$ odd. On the other hand, it follows from the Gysin sequence (10.1) that $H^{*}(S(k, l+k))$ is the direct sum of the kernel of $\cup \chi(V)$ with $\mathscr{A}(k, l)$ since both the middle terms in $(10.1)$ are free as modules over $\mathbb{Z}\left[b_{2}\right] /\left(b_{2}^{l}\right)$. Thus, the fact that the cokernel is known forces the structure of the kernel and Corollary 10.8 follows. q.e.d.

The following lemma is standard and is the second main tool needed for calculations.

Lemma 10.9. Let $\xi$ and $W$ be oriented bundles over a space $X$, and $T(\xi \oplus W)$ the associated Thom space of the Whitney sum. Then, in cohomology, the map induced by the inclusion $i: T(\xi) \subset T(\xi \oplus W)$ is given by

$$
i^{*}\left(a \cup U_{\xi \oplus W}\right)=a \cup \chi(W) \cup U_{\xi},
$$

where $\chi(W)$ is the Euler class of $W$.

We will be interested in the inclusion $\beta: N \rightarrow L$ where $N$ and $L$ are the sphere bundles associated to $\xi$ and $\xi \oplus W$ respectively. We can determine the cohomology map $\beta^{*}: H^{*}(L) \rightarrow H^{*}(N)$ using Lemma 10.9 and the long exact sequence

$$
\begin{aligned}
& \cdots \rightarrow H^{*}(X) \rightarrow H^{*}(L) \stackrel{\delta}{\rightarrow} H^{*+1}(T(\xi \oplus W)) \rightarrow \cdots \\
& \begin{array}{clc}
\downarrow & \downarrow \beta^{*} & \downarrow \cup(\chi(W)) \\
\ldots \rightarrow H^{*}(X) \rightarrow H^{*}(N) \stackrel{\delta}{\rightarrow} & H^{*+1}(T(\xi)) & \rightarrow \cdots
\end{array}
\end{aligned}
$$

since both extreme maps are known precisely.

To determine $H^{*}\left(\operatorname{Rat}_{1,1}\left(\mathbb{F}\left(j_{1}, j_{2}, n\right)\right)\right)$ we need to evaluate $\phi$ from (9.15). This we can do in two steps since the second matrix $\left(N_{2}\right)$ in our description of the points of $\operatorname{Rat}_{1,1}\left(\mathbb{F}\left(j_{1}, j_{2}\right)\right)$ is acted on by $U$ in $(9.7)$ and (9.17) but in no way contributes to $U$. More precisely, let $W\left(j_{1}, j_{2}\right)$ be the double mapping cylinder

$$
S\left(j_{1}, j_{2}\right) \stackrel{\bar{\phi}}{\longleftarrow}\left(S^{2 j_{1}-1} \times S^{2\left(j_{2}-j_{1}\right)-1}\right) / S^{1} \rightarrow \mathbb{C P}^{j_{1}-1} .
$$

Here $\bar{\phi}$ has the same description as given for $\phi$ in (9.15) and Proposition 9.16, except for ignoring the sphere $S^{2\left(n-j_{2}\right)-1}$. Then, up to homotopy 
type, we have the commutative diagram (10.12)

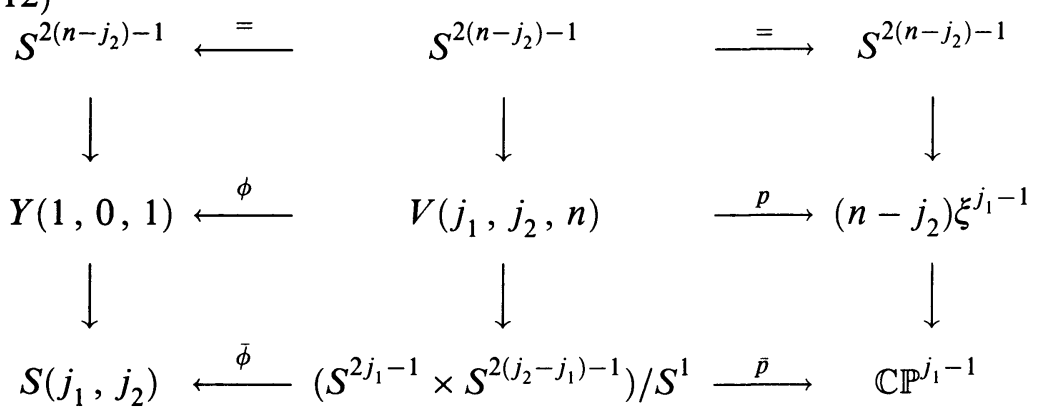

Here the leftmost vertical column is (10.6), the middle horizontal row is (9.15) and the bottom horizontal row is (10.11).

Consequently, since $W\left(j_{1}, j_{2}\right)$ and $\operatorname{Rat}_{(1,1)}\left(\mathbb{F}\left(j_{1}, j_{2}\right)\right)$ are, up to homotopy type, the double mapping cones the bottom and middle rows of (10.12) respectively, we may regard $\operatorname{Rat}_{1,1}\left(\mathbb{F}\left(j_{1}, j_{2}\right)\right)$ to be complement of the zero section in the fibration

$$
\mathbb{C}^{\left(n-j_{2}\right)} \rightarrow E \rightarrow W\left(j_{1}, j_{2}\right),
$$

with Euler class $-b_{2}^{n-j_{2}}$.

Therefore, we begin by determining $H^{*}\left(W\left(j_{1}, j_{2}\right)\right)$. In particular, (10.11) is equivalent to the existence of the exact sequence

$$
\begin{aligned}
\cdots \rightarrow H^{*}\left(T\left(\left(j_{2}-j_{1}\right) \xi_{j_{1}-1}\right)\right) & \rightarrow H^{*}\left(W\left(j_{1}, j_{2}\right)\right) \\
& \rightarrow H^{*}\left(S\left(j_{1}, j_{2}\right)\right) \stackrel{\delta}{\rightarrow} H^{*+1}\left(T\left(\left(j_{2}-j_{1}\right) \xi_{j_{1}-1}\right)\right) \rightarrow \cdots
\end{aligned}
$$

which shows $H^{*}\left(W\left(j_{1}, j_{2}\right)\right)$ is determined by the map $\delta$.

To explain the groups $H^{*}\left(W\left(j_{1}, j_{2}\right)\right)$ we need to introduce a new family of rings and modules.

\section{Definition 10.15.}

$$
\mathscr{B}(k, k+l)=\frac{\mathbb{Z}\left[b_{1}, b_{2}\right]}{\left(b_{1}^{k},\left(b_{1}-b_{2}\right) b_{2}^{l}, b_{1}^{l-2}\left(b_{1}^{2}-b_{2}^{2}\right)\right)} .
$$

Note that the relations imply that $b_{2}^{l+s}=b_{1}^{s} b_{2}^{l}$ for $s \geq 1$ and $b_{1}^{l+2 t}=$ $b_{2}^{2 t+2} b_{1}^{l-2}$ for $t \geq 0$. It follows that $b_{1}^{2 l-2+\varepsilon}=b_{2}^{2 l-2+\varepsilon}$ in $\mathscr{B}(k, k+l)$ and consequently $b_{2}^{\max (2 l-2+\varepsilon, k)}=0$. Indeed, the projection $\mathscr{B}(k, k+l) \rightarrow$ $\mathscr{A}(k, k+l)$ is onto with kernel $\left(b_{2}^{l+1}\right)=\mathbb{Z}\left(b_{2}^{l+1}, b_{2}^{l+2}, \cdots, b_{2}^{r}\right)$ where $r \leq \max (2 l-2+\varepsilon, k) . \mathscr{B}\left(j_{1}, j_{2}\right)$ is a direct summand of $H^{*}\left(W\left(j_{1}, j_{2}\right)\right)$ and we will shortly determine $r$, but first we introduce a module over $\mathscr{B}\left(j_{1}, j_{2}\right)$ which will turn out to be the remaining part of $H^{*}\left(W\left(j_{1}, j_{2}\right)\right)$. 
Definition 10.16. $\mathscr{N}_{a, l}$ is the module over $\mathscr{B}(k, k+l)$ on generators $h, \bar{s}$, of dimension $\operatorname{dim}(h)=0, \operatorname{dim}(\bar{s})=2(a+\varepsilon-1)$ and is specified by the relations

$$
\begin{gathered}
b_{1}^{l-1} h=0, \\
b_{2}^{\varepsilon} b_{1}^{a} h=\left(b_{1}+b_{2}\right) \bar{s}, \\
\left(b_{1}-b_{2}\right) b_{2}^{\varepsilon} p_{l-2-\varepsilon} h=b_{1}^{l-a} \bar{s} .
\end{gathered}
$$

Theorem 10.17. Let $k=j_{1}$ and $l=j_{2}-j_{1}$. Then $H^{*}\left(W\left(j_{1}, j_{2}\right)\right)$ is given as follows.

(a) When $k=2 l-2$ and $l$ is odd,

$$
H^{*}\left(W\left(j_{1}, j_{2}\right)\right)=\mathscr{B}\left(j_{1}, j_{2}\right) \oplus \overline{\mathscr{W}},
$$

where $\overline{\mathscr{W}}$ has generator $\bar{s}$ in dimension $4 l-3$ with relations $b_{1}^{l-2}$ $\cdot\left(b_{1}+b_{2}\right) \bar{s}=0, b_{2}^{l} \bar{s}=0$.

(b) If $l-2<k<2 l-2$ or $l$ is even, then

$$
H^{*}\left(W\left(j_{1}, j_{2}\right)\right)=\mathscr{B}\left(j_{1}, j_{2}\right) \oplus \Sigma^{2 k+3}\left(\mathcal{N}_{a, l}\right),
$$

where $a=\max (0,2 l-2-k)$.

(c) If $l$ is odd and $k>2 l-2$, then

$$
H^{*}\left(W\left(j_{1}, j_{2}\right)\right)=\mathscr{B}\left(j_{1}, j_{2}\right) \oplus \bar{W}^{\prime},
$$

where $\bar{W}^{\prime}$ has one generator, $\bar{s}$, in dimension $2 k+1$ with relations $b_{1}^{l-2}\left(b_{1}+b_{2}\right) \bar{s}=0, b_{2}^{l} \bar{s}=0$, and $b_{1}^{k} \bar{s}=0$.

Proof. Recall $S\left(j_{1}, j_{2}\right)=\left(S^{j_{1}-1} \times S^{2\left(j_{2}-j_{1}\right)-1} \times S^{2\left(j_{2}-j_{1}\right)-1}\right) / S^{1} \times S^{1}$ is the sphere bundle of $\xi_{j_{1}-1, j_{2}-j_{1}-1}^{-1,1} \oplus W$ over $\mathbb{C P}^{j_{1}-1} \times \mathbb{C P}^{j_{2}-j_{1}-1}$. Then (10.1) and (10.11) imply that Lemma 10.9 and (10.10) yield the following commutative diagram of long exact sequences:

$$
\begin{aligned}
& \cdots \rightarrow H^{*}\left(\mathbb{C P}^{j_{1}-1} \times \mathbb{C P}^{j_{2}-j_{1}-1}\right) \rightarrow \\
& \begin{array}{c}
\downarrow \\
\cdots \rightarrow H^{*}\left(\mathbb{C P}^{j_{1}-1} \times \mathbb{C P}^{j_{2}-j_{1}-1}\right) \rightarrow
\end{array} \\
& H^{*}\left(\frac{S^{2 j_{1}-1} \times S^{2\left(j_{2}-j_{1}\right)-1} \times S^{2\left(j_{2}-j_{1}\right)-1}}{S^{1} \times S^{1}}\right) \stackrel{\delta}{\rightarrow} H^{*}\left(T\left(\xi_{j_{1}-1, j_{2}-j_{1}-1}^{-1,} \oplus W\right)\right) \\
& \downarrow_{\bar{\phi}^{*}} \quad \downarrow i^{*} \\
& H^{*}\left(\frac{S^{2 j_{1}-1} \times S^{2\left(j_{2}-j_{1}\right)-1}}{S^{1}}\right) \quad \stackrel{\delta}{\rightarrow} \quad H^{*}\left(T\left(\xi_{j_{1}-1, j_{2}-j_{1}-1}^{-1,1}\right)\right)
\end{aligned}
$$

Since $i^{*}=\cup \chi(W)=\cup \pm b_{1}^{l-2}\left(b_{1}+b_{2}\right)$ it follows directly that $i^{*}\left(\Sigma^{2 k+3}(h)\right)$ $=0$ and that $i^{*}\left(\Sigma^{2 k+3}(s)\right) \neq 0$ in each of the cases (b) of Lemma 10.5. 
Next, when $k=2 l-2$ with $l$ odd, we see that $i^{*}(v)=i^{*}(w) \neq 0$, and when $k>2 l-2$ with $l$ odd, once more $i^{*}(s) \neq 0$. Indeed, all these images are integral generators, that is, they are not divisible by any integer except \pm 1 . On the other hand, by the exactness of the lower sequence, we see that the kernel of the map

$$
H^{*}\left(T\left(\xi_{j_{1}-1, j_{2}-j_{1}-1}^{-1,1}\right)\right) \stackrel{j^{*}}{\longrightarrow} H^{*}\left(\mathbb{C P}^{j_{1}-1} \times \mathbb{C P}^{j_{2}-j_{1}-1}\right),
$$

where $j^{*}$ is just $\cup \chi\left(\xi_{j_{1}-1, j_{2}-j_{1}-1}^{-1,1}\right)=\cup\left(-b_{1}+b_{2}\right)$, and is at most a single copy of $\mathbb{Z}$ in any dimension. It follows that $\bar{\phi}^{*}$ maps $s$ to an integral generator $H^{*}\left(\left(S^{2 j_{1}-1} \times S^{2\left(j_{2}-j_{1}\right)-1}\right) / S^{1}\right)$ in that dimension. Moreover, since $\bar{\phi}^{*}\left(b_{1}\right)=\bar{\phi}^{*}\left(b_{2}\right)=b$ is a generator in dimension 2 , it follows that all multiples $b^{i} \bar{\phi}^{*}(s)$ or $b^{i} \bar{\phi}^{*}(v)$ are also in the image. Hence, the map $\bar{\phi}^{*}$ is completely determined (and as nontrivial as it is possible for any such map to be). But now both maps in (10.11) are completely known in cohomology so that Corollary 10.8 and a Mayer-Vietoris computation on (10.11) establish the theorem. q.e.d.

Thus, $H^{*}\left(\operatorname{Rat}_{1,1}\left(\mathbb{F}\left(j_{1}, j_{2}\right)\right)\right)$ is obtained directly from Theorem 10.17 and the Gysin sequence applied to (10.13). In particular,

Theorem 10.18. If $n-j_{2} \geq j_{2}-j_{1}$, then

$$
H^{*}\left(\operatorname{Rat}_{1,1}\left(\mathbb{F}\left(j_{1}, j_{2}\right)\right)\right) \cong H^{*}\left(W\left(j_{1}, j_{2}\right)\right)\left(1, e_{2\left(n-j_{2}\right)-1}\right) .
$$

On the other hand, if $n-j_{2}<j_{2}-j_{1}$, then

$$
H^{*}\left(\operatorname{Rat}_{1,1}\left(\mathbb{F}\left(j_{1}, j_{2}\right)\right)\right) \cong \Sigma^{2\left(n-j_{2}\right)-1} \mathscr{K}\left(j_{1}, j_{2}, n\right) \oplus \mathscr{C}\left(j_{1}, j_{2}, n\right) .
$$

Here $\mathscr{H}\left(j_{1}, j_{2}, n\right)$ and $\mathscr{C}\left(j_{1}, j_{2}, n\right)$ denote the kernel and cokernel of

$$
\cup b_{2}^{n-j_{2}}: H^{*}\left(W\left(j_{1}, j_{2}\right)\right) \rightarrow H^{*}\left(W\left(j_{1}, j_{2}\right)\right) .
$$

Hence, they may be explicitly computed using Corollary 10.8 and Theorem 10.17 .

\section{On the homology of $\operatorname{Rat}_{C}(\mathbb{F}(J))$ above the range of stability}

In this section we explain why the homological behavior of arbitrary flag manifolds above the range of stability given in Theorem A is much more subtle than that for Grassmann manifolds. Recall from [11] that the natural inclusion $l_{n, m}$ always induces monomorphisms

$$
l(n, m ; k)_{t}: H_{t}\left(\operatorname{Rat}_{k}\left(\mathbb{G}_{n, m}\right) ; A\right) \rightarrow H_{t}\left(\Omega^{2} \mathbb{G}_{n, m} ; A\right)
$$


for all $k, n, m, t$, and all coefficients $A$. Furthermore, one can show that $\operatorname{Rat}_{1}\left(\mathbb{G}_{n, m}\right)$ is the basic building block for $H_{*}\left(\operatorname{Rat}_{k}\left(\mathbb{G}_{n, m}\right) ; A\right)$; that is, one can build Dyer-Lashof homology operations on $\bigsqcup_{k} \operatorname{Rat}_{k}\left(\mathbb{G}_{n, m}\right)$ and show that every class in $H_{*}\left(\operatorname{Rat}_{k}\left(\mathbb{G}_{n, m}\right) ; A\right)$ can be constructed from these operations applied to classes in $H_{*}\left(\operatorname{Rat}_{1}\left(\mathbb{G}_{n, m}\right) ; A\right)$, (see [12] for details). However, for all flag manifolds of length 2 or more this regular behavior fails to hold.

To illustrate this phenomenon we first compute $H_{*}\left(\Omega^{2} \mathbb{F}(J) ; \mathbb{Z} / p\right)$ and then use the results of the previous two sections to show that the natural inclusion $l(J ; \overrightarrow{1})$ has a nontrivial kernel in homology as long as the length of $J$ is at least 2 . Recall the flag manifold $\mathbb{F}(J)$ is given as the quotient of unitary groups

$$
\mathbb{F}(J)=U(n) /\left(U\left(j_{1}\right) \times U\left(j_{2}-j_{1}\right) \times \cdots \times U\left(n-j_{m}\right)\right) .
$$

The Stiefel manifold of $k$-frames in $\mathbb{C}^{n}$ is written $\mathbb{V}_{k, n-k}$ and is given as a quotient of unitary groups as $\mathbb{V}_{k, n-k}=U(n) / U(k)$. It is $2 k$-connected and there are fiberings $P_{i}$ given as

$$
V_{j_{i}-j_{i-1}, n-j_{i}+j_{i-1}} \rightarrow \mathbb{F}(J) \stackrel{P_{i}}{\longrightarrow} B_{U\left(j_{1}\right)} \times \cdots \times \widehat{B}_{U\left(j_{i}-j_{i-1}\right)} \times \cdots \times B_{U\left(n-j_{m}\right)}
$$

for each $i$ with $1 \leq i \leq m$.

The homology groups $H_{*}(U(j) ; \mathbb{Z})=E\left(e_{1}, e_{3}, \cdots, e_{2 j-1}\right)$ are well known, and

$$
H_{*}\left(\mathbb{V}_{j, n-j} ; \mathbb{Z}\right)=H_{*}(U(n) ; \mathbb{Z}) /\left(H_{*}(U(j) ; \mathbb{Z})\right) \cong E\left(e_{2 j+1}, \cdots, e_{2 n-1}\right) .
$$

Similarly, the work of Bott and Samelson, [3], determined the homology of the loop spaces here as

$$
\begin{aligned}
H_{*}(\Omega(U(n)) ; \mathbb{Z})=\mathbb{Z} \times & H_{*}(\Omega(\mathrm{SU}(n)) ; \mathbb{Z})=\mathbb{Z} \times \mathbb{Z}\left[e_{2}, \cdots, e_{2 n-2}\right], \\
H_{*}\left(\Omega \mathbb{V}_{r, n-r} ; \mathbb{Z}\right) & =H_{*}(\Omega(U(n)) ; \mathbb{Z}) /\left(H_{*}(\Omega(U(r)) ; \mathbb{Z})\right) \\
& =\mathbb{Z}\left[e_{2 r}, \cdots, e_{2 n-2}\right] .
\end{aligned}
$$

Lemma 11.2. Given $\mathbb{F}(J)$, let $v(J)=\max \left(j_{i}-j_{i-1}\right), 1 \leq i \leq m+1$, and suppose $i(J)$ is the first index $i$ so that $j_{i}-j_{i-1}=v(J)$. Let $A$ be any commutative ring. Then $H_{*}\left(\Omega^{2}(\mathbb{F}(J)) ; A\right)$ is isomorphic as a ring under loop-sum to the tensor product

$$
H_{*}\left(\Omega^{2}\left(\mathbb{V}_{v(J), n-j(J)}\right) ; A\right) \otimes_{A} H_{*}\left(\Omega\left(U\left(j_{1}\right)\right) ; \mathbb{Z}\right) \otimes \cdots \otimes H_{*}\left(\Omega\left(U\left(n-j_{m}\right)\right) ; \mathbb{Z}\right)
$$

with only the term $H_{*}(\Omega(U(v(J))) ; \mathbb{Z})$ deleted. 
Proof. Consider the Serre spectral sequence for the 2-fold looping of the fibration (11.1) where $i=i(J)$

$$
\begin{aligned}
\Omega^{2}\left(\mathbb{V}_{v(J), n-v(J)}\right) \rightarrow & \Omega^{2}(\mathbb{F}(J)) \\
& \stackrel{P_{i}}{\longrightarrow} \Omega\left(U\left(j_{1}\right)\right) \times \cdots \times \widehat{\Omega}(U(v(J))) \times \cdots \times \Omega\left(U\left(n-j_{m}\right)\right) .
\end{aligned}
$$

In homology the loop multiplications on the fiber, base, and total space determine an algebra structure in the spectral sequence so $E^{2}$, being the tensor product of $H_{*}$ (Base) $\otimes H_{*}$ (Fiber) (since $H_{*}$ (Base) is $\mathbb{Z}$-free), is also the tensor product of Base and Fiber as algebras. Moreover, the differentials are all derivations. It follows that the differentials are determined by their values on the generators on the base. But our choice of $i$ guarantees that the connectivity of the fiber is at least as large as the dimension of any generator on the base. Consequently, the differentials on these generators are identically zero and $E^{2}=E^{\infty}$.

Hence there are elements in $H_{*}\left(\Omega^{2}(\mathbb{F}(J)) ; A\right)$ which project onto the generators on the base. Since this is a second loop space (so the ring structure is commutative) and the homology of the base is a polynomial algebra on these generators, it follows that the subalgebra of $H_{*}\left(\Omega^{2}(\mathbb{F}(J)) ; A\right)$ generated by these elements is likewise the polynomial algebra on these generators. Next, the map Fiber $\times$ Total space $\rightarrow$ Total space induced by the loop-sum operation gives an explicit map of the tensor product of the fiber algebra with the base algebra onto the homology of the total space and completes the proof. q.e.d.

Thus, to complete our determination of $H_{*}\left(\Omega^{2}(\mathbb{F}(J)) ; A\right)$ it suffices to determine $H_{*}\left(\Omega^{2}\left(\mathbb{V}_{j, n-j}\right) ; A\right)$. We turn to this now. There is a spectral sequence (the cobar spectral sequence derived from [1]) with $E_{2}^{s, t}$-term $\operatorname{Ext}_{H^{*}\left(X ; \mathbb{F}_{p}\right)}^{s, t}\left(\mathbb{F}_{p}, \mathbb{F}_{p}\right)$ that converges to $H_{t-s}\left(\Omega X ; \mathbb{F}_{p}\right)$. These Ext-groups are calculated as follows:

$$
\begin{aligned}
\operatorname{Ext}_{A \otimes B}\left(\mathbb{F}_{p}, \mathbb{F}_{p}\right) & =\operatorname{Ext}_{A}\left(\mathbb{F}_{p}, \mathbb{F}_{p}\right) \otimes \operatorname{Ext}_{B}\left(\mathbb{F}_{p}, \mathbb{F}_{p}\right), \\
\operatorname{Ext}_{\mathbb{F}_{p}[x] / x^{p}}\left(\mathbb{F}_{p}, \mathbb{F}_{p}\right) & =\mathbb{E}(|x|) \otimes \mathbb{F}_{p}\left[\gamma_{x}^{i}\right],
\end{aligned}
$$

where $|x|$ has bidegree $(1, \operatorname{dim}(x))$ and $\gamma_{x}^{i}$ has bidegree $\left(2, p^{i} \cdot \operatorname{dim}(x)\right)$. For example the $E_{2}$-term for $\Omega(\operatorname{SU}(3))$ at 2 is

$$
\mathbb{E}\left(\left|e_{2}\right|\right) \otimes \mathbb{E}\left(\left|e_{2}^{2}\right|, \cdots,\left|e_{2}^{2^{i}}\right|, \cdots\right) \otimes \mathbb{F}_{p}\left[\gamma_{e_{2}}^{2}, \cdots, \gamma_{e_{2}^{2^{i}}}^{2}, \cdots\right]
$$

and $E_{2}$ equals $E_{\infty}$.

We consider $\Omega \mathrm{SU}(n)$ and $\Omega \mathbb{V}_{r, n-r}$ for $r>1 . \quad H_{*}(\Omega \mathrm{SU}(n) ; \mathbb{Z})=$ $\mathbb{Z}\left[e_{2}, e_{4}, \cdots, e_{2 n-2}\right]$ is a commutative and cocommutative Hopf algebra 
with $\psi\left(e_{2 j}\right)=\sum e_{2 k} \otimes e_{2(j-k)}$ since there is a map

$$
\lambda: \mathbb{C P}^{n-1} \rightarrow \Omega \mathrm{SU}(n)
$$

so that $\lambda\left(e^{2 j} \cap\left[\mathbb{C P}^{n-1}\right]\right)=e_{2 n-2 j-2}$ for $j=0,1, \cdots, n-2$. Also,

$$
H_{*}\left(\Omega \mathbb{V}_{r, n-r} ; \mathbb{Z}\right)=H_{*}(\Omega U(n) ; \mathbb{Z}) / / H_{*}(\Omega U(r) ; \mathbb{Z})=\mathbb{Z}\left[e_{2 r}, \cdots, e_{2 n-2}\right],
$$

the quotient Hopf algebra. (For details see [15].)

The dual algebras $H^{*}\left(\Omega S U(n) ; \mathbb{F}_{p}\right)$ and $H^{*}\left(\Omega \mathbb{V}_{r, n-r} ; \mathbb{F}_{p}\right)$ are tensor products of algebras of the form

$$
\mathbb{F}_{p}\left[\theta_{2 j}\right] / \theta_{2 j}^{p^{k(j)}} \otimes \mathbb{F}_{p}\left[\theta_{2 p j}\right] / \theta_{2 p j}^{p^{k(j)}} \otimes \cdots \otimes \mathbb{F}\left[\theta_{2 p^{r} j}\right] / \theta_{2 p^{r} j}^{p^{k(j)}} \otimes \cdots
$$

for $1 \leq j \leq n-1$, in the case of $\mathrm{SU}(n)$, and $r \leq j \leq n-1$ for $\mathbb{V}_{r, n-r}$, where $j$ and $p$ are relatively prime. Here $k(j)$ is given by $[n / j]=p^{r(j)} w$ with $w$ prime to $p$, and $[a]$ denotes the integer part of $a$. In both the cases of $\mathrm{SU}(n)$ and of $\mathbb{V}_{r, n-r}$ the spectral sequences collapse, so $E_{2}=E_{\infty}$ for all $p$, which determines $H_{*}\left(\Omega^{2} \mathrm{SU}(n) ; \mathbb{F}_{p}\right)$ and $H_{*}\left(\Omega^{2} \mathbb{V}_{r, n-r} ; \mathbb{F}_{p}\right)$.

This is a more efficient way to compute $H_{*}\left(\Omega^{2} \mathbb{F}(J)\right)$ than by using the Serre spectral sequences associated to the fibration in the right-hand column of (7.4). However, this direct computation is not as useful as (7.7) when studying flags, and thus the computation given above and a comparison argument need to be used to compute the differentials on the right-hand side of (7.7). This proceeds as follows:

Rationally we have

$$
H_{*}\left(\Omega^{2}\left(\mathbb{V}_{j, n-j}\right) ; \mathbb{Q}\right) \cong \operatorname{Ext}_{\mathbb{Q}\left[e_{2 j}, \cdots, e_{2 n-2}\right]}(\mathbb{Q}, \mathbb{Q}) \cong E\left(e_{2 j-1}, \cdots, e_{2 n-3}\right),
$$

from which it follows that, when we factor out the torsion direct summand, we have $H_{*}\left(\Omega^{2}\left(\mathbb{V}_{j, n-j}\right) ; \mathbb{Z}\right) /($ torsion $)=E\left(e_{2 j-1}, \cdots, e_{2 n-3}\right)$ as well. In the case of the Grassmannians $\mathbb{G}_{j, n-j}=U(n) /(U(j) \times U(n-j))$ we can assume $n-j \geq j$, that is when $v(J)=n-j$. Then the torsion free generators are exactly $\left\langle e_{2}, \cdots, e_{2 j-2}, f_{2 n-2 j-1}, \cdots, f_{2 n-3}\right\rangle$, and those are in one-to-one correspondence with the elements of $\operatorname{Rat}_{1}\left(\mathbb{G}_{j, n-j}\right)$. Thus, we can expect things to be considerably simpler here than in the case of the general flag manifold. In fact

$$
\begin{aligned}
H_{*}\left(\Omega_{0}^{2}\left(\mathbb{G}_{j, n-j}\right) ; \mathbb{Z} / p\right)= & \mathbb{Z} / p\left[b_{2}, \cdots, b_{2 j-2}\right] \\
& \otimes \operatorname{Cotor}_{E\left(e_{2 n-2 j}, \cdots, e_{2 n-2}\right)}(\mathbb{Z} / p, \mathbb{Z} / p) \\
= & \mathbb{Z} / p\left[b_{2}, \cdots, b_{2 j-2}\right] \otimes H_{*}\left(\Omega^{2} S^{2 n-2 j+1} ; \mathbb{Z} / p\right) \\
\otimes & \otimes \otimes H_{*}\left(\Omega^{2} S^{2 n-1} ; \mathbb{Z} / p\right),
\end{aligned}
$$


and this isomorphism is both as rings (loop sum), and modules over the Dyer-Lashof operations. Here $H_{*}\left(\Omega^{2} S^{2 s+1} ; \mathbb{Z} / p\right)$ is described as follows. There is a (torsion free) generator $f_{2 s-1} \in H_{2 s-1}\left(\Omega^{2} S^{2 s+1} ; \mathbb{Z} / p\right)=\mathbb{Z} / p$, and for $p$ any odd prime

$$
\begin{aligned}
H_{*}\left(\Omega^{2} S^{2 s+1} ; \mathbb{Z} / p\right)= & E(f, Q_{1}(f), \cdots, \underbrace{Q_{1} \cdots Q_{1}}_{i \text { times }}(f), \cdots) \\
& \otimes \mathbb{Z} / p[\beta Q_{1}(f), \cdots, \beta \underbrace{Q_{1} \cdots Q_{1}}_{i \text { times }}(f), \cdots]
\end{aligned}
$$

where $Q_{1}$ is the first Dyer-Lashof operation (it takes a class of dimension $l$ to a class of dimension $l p+p-1)$, and $\beta$ is the $\bmod (p)$ Bochstein. With $\bmod (2)$ coefficients the form of the answer is slightly different, as follows:

$$
H_{*}\left(\Omega^{2} S^{2 s+1} ; \mathbb{Z} / 2\right)=\mathbb{Z} / 2[f, Q_{1}(f), \cdots, \underbrace{Q_{1} \cdots Q_{1}}_{i \text { times }}(f), \cdots],
$$

and $\beta\left(Q_{i}(f)\right)=f^{2}$.

Now proceed by induction. Let $J=\left(j_{1}, j_{2}, \cdots, j_{m}\right)$ and set $J^{\prime}=$ $\left(j_{2}, \cdots, j_{m}\right)$. Then the differentials in the Serre spectral sequence for

$$
\Omega^{2} \mathbb{G}_{j_{1}, j_{2}-j_{1}} \rightarrow \Omega^{2} \mathbb{F}(J) \rightarrow \Omega^{2} \mathbb{F}\left(J^{\prime}\right)
$$

with $\bmod (p)$ coefficients are as follows. The $E^{2}$-term is the tensor product of the groups described in Lemma 11.2 with those of (11.3), and the generating differentials are transgressive with $\partial\left(e_{2 s}(i)\right)=f_{2 s-1}$ anytime both $e_{2 s}$ and $f_{2 s-1}$ are nonzero. There are also transgressive differentials from the base on the terms in $H_{*}\left(\mathbb{V}_{v\left(J^{\prime}\right), n-v\left(J^{\prime}\right)} ; \mathbb{Z} / p\right)$ :

$$
\begin{aligned}
\partial\left(e_{4 s-1}\right) & =e_{2 s-1} * e_{2 s-1}, \quad \text { with } \mathbb{Z} / 2 \text { coefficients, } \\
\partial\left(e_{2 s p-1}\right) & =\beta Q_{1}\left(e_{2 s-1}\right), \quad \text { with } \mathbb{Z} / p \text { coefficients. }
\end{aligned}
$$

These have the effect in the $\bmod (2)$ case of building a (torsion free) generator in the total space represented by $Q_{1}\left(e_{2 s-1}\right)$ and having image $2 e_{4 s-1}$ on the base. Similar things happen for odd primes.

With this background we now determine the kernel of

$$
l_{*}: H_{*}\left(\operatorname{Rat}_{1, \ldots, 1}(\mathbb{F}(J))\right) \rightarrow H_{*}\left(\Omega^{2} \mathbb{F}(J)\right) .
$$


In this case the $E^{2}$ term in Theorem $7.5, E^{2}\left(\operatorname{Rat}_{\overrightarrow{1}}(\mathbb{F}(J))\right)$, has two direct summands:

$$
\widetilde{H}_{*}\left(\operatorname{Rat}_{1}\left(\mathbb{G}_{j_{1}, j_{2}-j_{1}}\right) ; A\right) \otimes H_{*}\left(\operatorname{Rat}_{\overrightarrow{1}}\left(\mathbb{F}\left(J^{\prime \prime}\right)\right) ; A\right)
$$

and

$$
H_{*}\left(\operatorname{Rat}_{\overrightarrow{1}}\left(\mathbb{F}\left(J^{\prime}\right)\right) ; A\right),
$$

where $J^{\prime}=\left(j_{1}, \cdots, j_{m}\right), J^{\prime \prime}=\left(j_{2}-j_{1}, \cdots, j_{m}-j_{1}\right)$, and $A$ are arbitrary (untwisted) coefficients. The first summand consists of all terms whose multipartition $K$ has $k_{1}(1)=1$ while the second summand consists of all terms where $k_{1}(1)=0$. In particular, for $\operatorname{Rat}_{1,1}\left(\mathbb{F}\left(j_{1}, j_{2}, n\right)\right)$ this $E^{2}$ term becomes

$$
\begin{aligned}
\left(\widetilde{H}_{*}\left(\operatorname{Rat}_{1}\left(\mathbb{G}_{j_{1}, j_{2}-j_{1}}\right) ; A\right)\right. & \left.\otimes H_{*}\left(\operatorname{Rat}_{1}\left(\mathbb{G}_{j_{2}-j_{1}, n-j_{2}}\right) ; A\right)\right) \\
& \oplus H_{*}\left(\operatorname{Rat}_{1}\left(\mathbb{G}_{j_{2}, n-j_{2}}\right) ; A\right) .
\end{aligned}
$$

When we compare (11.6) with the results of $\S 10$ we see that for $j_{2}-j_{1} \geq j_{1}$ there is an "internal" differential (staying in the first summand in (11.6)),

$$
d\left(e_{2\left(j_{2}-j_{1}\right)-1}\right)=b_{1}^{j_{2}-j_{1}}-b_{1}^{j_{2}-j_{1}-2} b_{2}^{2}=-b_{1}^{j_{2}-j_{1}-2} b_{2}^{2},
$$

and an external differential as well,

$$
d\left(b_{2}^{j_{2}-j_{1}-2} e_{2\left(j_{2}-j_{1}\right)-1}\right)=b_{2}^{2\left(j_{2}-j_{1}\right)-2},
$$

going from the first to the second summand in (11.6).

If $n-j_{2} \geq j_{2}$, then it is direct to verify that (11.6) injects into the $E^{2}$-term of the Serre spectral sequence for $\Omega^{2} \mathbb{F}\left(j_{1}, j_{2}, n\right)$ given in (7.7). In this case set $m=\max \left(j_{2}-j_{1}, j_{1}\right)$. Then the results of $\S 10$ or naturality of differentials in the dual spectral sequence imply $\delta\left(e_{2 m-1}^{*}\right)=\left(b_{2}^{m}\right)^{*}$ on the dual classes in cohomology. However, since $\left(b_{1}-b_{2}\right) b_{2}^{m}=0$ in $H_{*}\left(\operatorname{Rat}_{1,1}\left(\mathbb{F}\left(j_{1}, j_{2}, n\right)\right)\right)$, the cohomology class dual to $\left(b_{1}-b_{2}\right) e_{2 m-1}$ must be an infinite cycle which is not in the image from the $E_{\infty}$-term of the Serre spectral sequence for $H^{*}\left(\Omega^{2} \mathbb{F}\left(j_{1}, j_{2}, n\right)\right)$. Note we can pass freely between homology and cohomology here as (11.6) is torsion free. By passing to rational coefficients it is direct to verify that no multiple of the dual of $\left(b_{1}-b_{2}\right) e_{2 m-1}$ is in the image. Thus, the map in cohomology is not surjective, and therefore the map $i_{*}$ in homology is not injective.

Similarly, when $n-j_{2}<j_{2}$ we have two possibilities, $j_{2}-j_{1}<n-j_{2}$ or $n-j_{2}<j_{2}$. In either case, the classes $e_{2\left(j_{2}-j_{1}\right)-1} b_{2}^{l}$ for $l<n-j_{2}$ occur in the main block and are infinite cycles. When $j_{2}-j_{1}<n-j_{2}$, the classes $e_{j_{2}} b_{2}^{l}$ in the smaller part of the Leray spectral sequence for 
$\operatorname{Rat}_{(1,1)}(\mathbb{F}(J))$ are the terms in the image from the Serre spectral sequence. Consequently, here again the cohomology map is not surjective, so the map $l_{*}$ in homology has a kernel. The final case where $n-j_{2}<j_{2}$ is almost identical; only the indices on the $e_{*}$ classes change slightly. Thus, in all cases of 2-stage flags, the homology map $l_{*}$ on $H_{*}\left(\operatorname{Rat}_{1,1}(\mathbb{F}(J)) ; \mathbb{Z}\right)$ has a kernel which is a direct summand. It is now clear that the same result extends directly for all $\operatorname{Rat}_{\hat{1}}(\mathbb{F}(J))$ where $J$ has 3 or more terms. It is direct to extend the Dyer-Lashof construction given in [12] for $\operatorname{Rat}\left(\mathbb{G}_{n, m}\right)$ to $\operatorname{Rat}(\mathbb{F}(J))$, and we believe that this Dyer-Lashof construction propagates these kernel classes to all the $\operatorname{Rat}_{C}(\mathbb{F}(J))$. However, it appears to be relatively difficult to verify that these $Q_{1}(v)$-classes with $v \in \operatorname{Ker}\left(l_{*}\right)$ are, in fact, nonzero in $H_{*}\left(\operatorname{Rat}_{C}(\mathbb{F}(J)) ; \mathbb{Z} / p\right)$. But modulo this we are convinced that there are kernels for all the homology maps $H_{*}\left(\operatorname{Rat}_{C}(\mathbb{F}(J)) ; \mathbb{Z} / p\right) \rightarrow H_{*}\left(\Omega^{2} \mathbb{F}(J) ; \mathbb{Z} / p\right)$ as long as $J$ has two or more nonzero terms.

Finally, it can be shown that the Dyer-Lashof operations and $\mathbb{Z} \stackrel{p}{\rightarrow}$ $\mathbb{Z} \rightarrow \mathbb{Z} / p$ Bochsteins do not generate the homology of $\Omega^{2} \mathbb{V}_{r, n-r}$ from the "spherical" classes coming from the $\operatorname{Rat}_{\hat{1}}(\mathbb{F}(J))$ in the case when $n-r>r$. Essentially what happens is that higher Bochsteins connect the $Q_{1} \cdots Q_{1}(e)$ Dyer-Lashof classes to various polynomial generators when enough $Q_{1}$ 's are present for certain choices of the $e$ 's. Thus, for all flags where the length of $J$ is at least two, in order to generate all of $H_{*}\left(\Omega^{2} \mathbb{F}(J)\right)$ under the image of $l_{*}$ (which we know can eventually be done because of the stability theorem) one must use more than operations on the classes from $H_{*}\left(\operatorname{Rat}_{\overrightarrow{1}}(\mathbb{F}(J))\right.$.

\section{Bibliography}

[1] J. F. Adams, On the cobar construction, Proc. Nat. Acad. Sci. U.S.A. 42 (1956) 409-412.

[2] C. P. Boyer, J. C. Hurtubise, B. M. Mann \& R. J. Milgram, The topology of instanton moduli spaces. I: the Atiyah-Jones conjecture, Ann. of Math, to appear.

[3] R. Bott \& H. Samelson, Applications of the theory of Morse to symmetric spaces, Amer. J. Math. 80 (1958) 964-1029.

[4] J. M. C. Clark, The consistent selection of parameterizations in system identification, Proc. Joint Automatic Control Conf., 1976, 576-580.

[5] F. R. Cohen, R. L. Cohen, B. M. Mann \& R. J. Milgram, The topology of rational functions and divisors of surfaces, Acta Math. 166 (1991) 163-221.

[6] D. F. Delchamps, Global structures of families of multivariable systems with an application to identification, Math. Systems Theory 18 (1985) 329-380.

[7] M. A. Guest, Topology of the space of absolute minima of the energy functional, Amer. J. Math. 106 (1984) 21-42.

[8] J. C. Hurtubise, The classification of monopoles for the classical groups, Comm. Math. Phys. 120 (1989) 613-641. 
[9] J. C. Hurtubise \& M. K. Murray, On the construction of monopoles for the classical groups, Comm. Math. Phys. 122 (1989) 35-89.

[10] F. C. Kirwan, On spaces of maps from Riemann surfaces to Grassmannians and applications to the cohomology of moduli of vector bundles, Ark. Mat. 24 (1986) 221-275.

[11] B. M. Mann \& R. J. Milgram, Some spaces of holomorphic maps to complex Grassmann manifolds, J. Differential Geometry 33 (1991) 301-324.

[12] _ - The topology of rational maps to Grassmannians and a homotopy theoretic proof of the Kirwan stability theorem, Algebraic Topology, Oaxtepec, 1991, Contemporary Math., vol. 146, Amer. Math. Soc., Providence, RI, 1993, 251-275.

[13] M. K. Murray, Stratifying monopoles and rational maps, Comm. Math. Phys. 125 (1989) 661-674.

[14] G. Segal, The topology of rational functions, Acta Math. 143 (1979) 39-72.

[15] N. E. Steenrod \& D. B. A. Epstein, Cohomology operations, Princeton Univ. Press, Princeton, NJ, 1962.

University of New MeXico STANFORD UNIVERSITY 
